\title{
Estrutura e exemplos de $A$-Loops comutativos finitos
}

\author{
Dylene Agda Souza de Barros
}

DissertaÇÃo APRESENTADA

$\mathrm{AO}$

Instituto De Matemática e Estatística

DA

Universidade de SÃo Paulo

PARA

OBTENÇÃO DO TÍTULO

$\mathrm{DE}$

Mestre em CiÊnCIAS

\author{
Programa: Matemática \\ Orientador: Prof. Dr. Alexandre Grichkov
}

Durante o desenvolvimento deste trabalho o autor recebeu auxílio financeiro da CAPES/CNPq

São Paulo, fevereiro de 2010 



\title{
Estrutura e exemplos de A-loops comutativos finitos
}

\author{
Esta versão definitiva da dissertação \\ contém as correções e alterações sugeridas pela \\ Comissão Julgadora durante a defesa realizada \\ por Dylene Agda Souza de Barros em 03/03/2010.
}

Comissão Julgadora:

- Prof. Dr. Alexandre Grichkov (orientador) - IME-USP

- Prof. Dr. Henrique Guzzo Junior - IME-USP

- Prof. Dr. Alexandr Zubkov - OMSK STATE 

Dedico esse trabalho à minha sobrinha Sarah, in memorian. 



\section{Agradecimentos}

Para mim é impossível começar esse trabalho sem agradecer a Deus por... tudo. Quero agradecer aos meus pais, Raimundo e Maria Creuza, por me darem amor e suporte para correr atrás dos meus sonhos, e aos meus irmãos, Marco, Marcelo, Denyse e Matheus, simplismente por fazerem parte da minha vida, contribuindo, cada um da sua forma, com a pessoa que sou. Um especial muito obrigada a minha irmã Denyse por, mesmo com a distância, estar sempre tão presente.

Quero agradecer aos meus professores da gradução do IME-UFG, em especial à professora Shirlei, ao professor Romildo e ao professor Walterson, por todas as lições dadas. Todas elas me ajudaram muito durante o mestrado. Falando em IME-UFG, tenho aqui que lembrar dos meus amigos queridos Marcelo, Poliana, Thaynara, Bira, Eduardo, Arianny, Adriana, Lidiane, Wender, Sunamita, Tarcísio, Vanessa e, é claro, da minha flor de Canella, Ju. Eu sinto muita saudade de todos vocês.

Muito obrigada a todos os funcionários e professores do IME-USP, por me receberem tão bem, em especial ao meu orientador, professor Alexandre Grichkov, por tanta paciência e por todos os ensinamentos durante esses dois anos.

Aos meus amigos do IME-USP, Gustavo, Rose, Diego, Maurício, Débora, Humberto, Juliano, Graciele, Taty, Fran, Big, Bruno, Arlane, André, Bernardo, eu não sei nem o que dizer para agradecer. Vocês fizeram toda a diferença nesses dois anos, desde a piada sem graça que mesmo assim alegra ao silêncio que compreende, passando sempre pelo abraço que conforta. Meus dias aqui foram mais felizes por causa de vocês... 



\section{Resumo}

Esse trabalho trata um pouco da teoria de $A$-loops comutativos finitos.

No primeiro capítulo estudamos propriedades básicas de loops em geral e exibimos exemplos de loops não associativos. No capítulo 2 falamos de $A$-loops em geral e mesmo sem assumirmos comutatividade obtivemos resultados importantes, um exemplo é que $A$-loop associa potências. Também determinamos quando um isótopo e $K^{*}$-holomorfo de um $A$-loop é um $A$-loop.

No capítulo 3 , nossos únicos objetos de estudo foram os $A$-loops comutativos finitos. Vimos que tais estruturas têm proriedades muito interessantes, por exemplo, para um $A$-loop comutativo finito valem os teoremas de Lagrange, Cauchy. Também, um $A$-loop comutativo finito, $Q$, tem ordem potência de um primo $p$ se e somente se todo elemento de $Q$ tem ordem potência de $p$. Mais ainda, todo $A$-loop comutativo finito de ordem ímpar é solúvel. No último capítulo, apresentamos algumas maneira de se construir um A-loop.

Palavras-chave: $A$-loops, Aplicações internas, Decomposição, $A$-loops comutativos de oredem ímpar, $A$-loops comutativos de expoente 2, Extensões centrais, $A$-loops comutativos de ordem $p^{3}$. 



\section{Abstract}

This work is about finite commutative $A$-loops.

In the first chapter we studied basic properties of general loops and we showed some examples of nonassociative loops. In chapter 2 , we talked about general $A$-loops (without commutativity) and even that we obtained important results, for instance, that any $A$-loop is power-associative. We also determined when an isotope and a $K^{*}$-holomorph of an $A$-loop is an $A$-loop.

In chapter 3 we dealt only with finite commutative $A$-loops. We saw that such structures have very interesting properties, for example, for a finite commutative $A$ loop, Lagrange, Cauchy's theorems apply. Also a finite commutative $A$-loop, $Q$, has order a power of a prime $p$ if and only if every element of $Q$ has order a power of $p$. Moreover, finite commutative $A$-loops of odd order are solvable. In the last chapter we introduce some ways to construct a commutative $A$-loop

Keywords: $A$-loops, Inner mappings, Decomposition, Commutative $A$-loops of odd order, Commutative $A$-loops of exponent 2, Central extension, Commutative $A$-loop of order $p^{3}$. 



\section{Sumário}

1 Loops 17

1.1 Conceitos e Proriedades Básicas . . . . . . . . . . . . . . . 17

1.2 Loops com a Propriedade do Inverso . . . . . . . . . . . . . . 26

1.3 Exemplos de Loops . . . . . . . . . . . . . . . . . . . . . 29

2 A-loops 33

2.1 Propriedades Gerais . . . . . . . . . . . . . . . . . . 33

2.2 A-loops Diassociativos . . . . . . . . . . . . . . . 41

2.3 Isotopias de $A$-Loops . . . . . . . . . . . . . . . . . . . . . . . . . . 45

2.4 Holomorfos de $A$-loops . . . . . . . . . . . . . . . . . . . . . . 50

2.5 Construção e Exemplos de A-Loops . . . . . . . . . . . . . . . . . 53

3 A-Loops Comutativos Finitos: Estrutura $\quad 59$

3.1 Notações e Fatos Básicos . . . . . . . . . . . . . . . 59

3.2 A-loops Comutativos Finitos de Ordem Ímpar . . . . . . . . . . . . . 64

3.3 Quadrados e Loop Associado . . . . . . . . . . . . . . . 73

3.4 O Teorema de Decomposição . . . . . . . . . . . . . . . . . 77

3.5 A-Loops Comutativos de Expoente $2 \ldots \ldots$. . . . . . . . 82

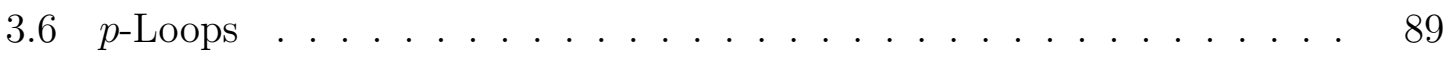

4 A-Loops Comutativos Finitos: Construções $\quad 91$

4.1 Loops comutativos cujo núcleo intermediário tem índice 2 . . . . . . . 91 
4.2 Construções de $A$-Loops Comutativos com Núcleo Intermediário de Índice $2 \ldots \ldots \ldots \ldots 10 \ldots$

4.2.1 A-loops comutativos de ordem $8 \ldots \ldots$. . . . . . . . . 101

4.2.2 Uma classe de $A$-loops comutativos de expoente 2 com centro trivial e núcleo intermediário de índice 2 . . . . . . . . . . . 103

4.3 Extensões Centrais Baseadas em Formas Trilineares . . . . . . . . . . 104 4.3.1 Somando Cociclos de Grupos . . . . . . . . . . . . . . 108

4.4 Uma Classe de $A$-Loops Comutativos de Ordem $p^{3} \quad \ldots \ldots$. . . . . 109

5 Apêndice: $A$-Loops Comutativos Nilpotentes de Grau $2 \quad 116$ 




\section{Capítulo 1}

\section{Loops}

\subsection{Conceitos e Proriedades Básicas}

Daremos, neste capítulo, a definição, exemplos e algumas propriedades básicas de loops.

Definição 1.1.1. Um conjunto não vazio L munido de uma operação binária é um quase-grupo se as equações $a x=b, x a=b$ têm uma única solução em $L$ quaisquer que sejam $a, b \in L$.

Um loop é um quase-grupo $L$ tal que existe $1 \in L$ com a propriedade que

$$
1 a=a 1=a \quad \text { para todo } \quad a \in L .
$$

Salvo menção contrária, $L$ denotará um loop.

Dado $a \in L$, a equação $a x=1$ tem única solução $x=a^{\rho}$ que é chamado de inverso à direita de $a$; da mesma forma a equação $x a=1$ tem única solução $x=a^{\lambda}$ que é chamado de inverso à esquerda de $a$. Se em $L$ valer a propreidade associativa, obtemos um grupo, pois $a^{\rho}=a^{\lambda}$ para todo $a \in L$. Em outras palavras, um grupo é um loop associativo.

Notemos que se $a, b, c \in L$ são tais que $a b=a c=d$, pela unicidade da solução da 
equação $a x=d$ temos que $b=c$, e se, por outro lado, $b a=c a=f$ temos, pelo mesmo argumento, que $b=c$. Ou seja, em um loop $L$ vale a lei do cancelamento.

Seja $a \in L$ e defina $R_{a}: L \longrightarrow L$ por $R_{a}(x)=x a$ e $L_{a}: L \longrightarrow L$ por $L_{a}(x)=$ ax. Temos que $R_{a}$ e $L_{a}$ são bijeções de $L$ e consideremos $\mathcal{M}(L)=\left\langle R_{x}, L_{x} \mid x \in L\right\rangle$ subgrupo de $\mathcal{A}(L)$ chamado grupo das multiplicações de $\boldsymbol{L}$, onde $\mathcal{A}(L)$ é o grupo das permutações do conjunto $L$.

Definição 1.1.2. Um subconjunto não vazio $H \subseteq L$ é um subloop se, com a operação de $L$ restrita a $H, H$ tiver estrutura de loop.

Note que, se $H \subseteq L$ for um subloop, a equação $h x=h$, onde $h \in H$, tem única solução em $H$ e em $L$. Portanto $1_{H}=1_{L}$.

Proposição 1.1.3. Seja $H \subseteq L$ un subconjunto não vazio de um loop L. São equivalentes:

a) $H$ é um subloop de $L$;

b) Se $x, y \in H$, entãa $x y, R_{y}^{-1}(x)$ e $L_{y}^{-1}(x)$ estão em $H$;

c) Se $x, y, z \in L$ com $x y=z$ e dois destes elementos estão em $H$, então o terceiro elemneto também está em $H$.

Demonstração. Vamos supor verdadeiro o ítem a, isto é $H$ subloop de $L$ e sejam $x, y \in H$. Como $H$ é fechado pela operação de L, temos $x y \in H$. Temos que $z=R_{y}^{-1}(x) \in L$ é a única solução da equação $z y=x$, portanto está em $H$. Da mesma maneira, $w=L_{y}^{-1}(x)$ está em $H$ pois é a inica solução da equação yw $=x$. Agora supondo o item $b$, sejam $x, y, z \in L$ com $x y=z$. Se $x, y \in H$, segue que $z \in H$. Se $x, z \in H$ temos $y=L_{x}^{-1}(z) \in H$ e se $y, z \in H$ temos $x=R_{y}^{-1}(z) \in H$. Finalmente, supondo o item c sejam $x, y \in H$. Então temos $z=x y \in H$. Como existem únicos $w, v \in L$ tais que $x w=y$ e $v x=y$ seguem e que $w, v \in H$ e então $H$ é um quase-grupo. Finalmente para todo $x \in H, \quad 1 x=x$ donde segue que $1 \in H$. Logo $H$ é um subloop de $L$, como queíamos demonstrar.

Notemos que se $H$ for um subloop de $L$, então $a^{\rho}$ e $a^{\lambda}$ estão em $H$ para todo $a \in H$. 
Definição 1.1.4. Sejam $H$ um subloop do loop $L$ e $x \in L$. Os conjuntos $H x=$ $\{h x: \in H\}$ e $x H=\{x h: \in H\}$ são chamados, de classe lateral à direita de $\boldsymbol{H}$ por $\boldsymbol{x}$ e classe lateral à esquerda de $\boldsymbol{H}$ por $\boldsymbol{x}$.

Suponha, $H$ subloop de $L$ e $x, y \in L$ tais que $H x=H y$. Então para qualquer que seja $h \in H$, existem $h_{1}, h_{2} \in H$ tais que $h x=h_{1} y$ e $h y=h_{2} x$, isto é, $x=$ $L_{h}^{-1}\left(L_{h_{1}}(y)\right)$ e $y=L_{h}^{-1}\left(L_{h_{2}}(x)\right)$. Reciprocamente se $x, y \in L$ são tais que, para todo $h \in L$ existem $h_{1}, h_{2} \in H$ satisfazendo $x=L_{h}^{-1}\left(L_{h_{1}}(y)\right)$ e $y=L_{h}^{-1}\left(L_{h_{2}}(x)\right)$, então $H x=H y$. Analogamente $x H=y H$ se e somente se, para todo $h \in H$ existem $h_{1}, h_{2} \in H$ tais que $x=R_{h}^{-1}\left(R_{h_{1}}(y)\right)$ e $y=R_{h}^{-1}\left(R_{h_{2}}(x)\right)$. Assim temos que $x H=H=1 H$ e $H x=H=H 1$ se e somente se $x \in H$

Existe uma bijeção natural ente $H x$ e $H$. Daí temos que $|H|=|H x|$ para todo $x \in L$. Da mesma forma $|H|=|x H|$ para todo $x \in H$. Então temos que, se $L$ for um loop finito e puder ser escrito como reunião disjunta de classes laterais à direira (ou à esquerda) do subloop $H$, a ordem de $H$ divide a ordem de $L$.

Proposição 1.1.5. Sejam $L$ um loop, $H$ um subloop de $L$ e $x, y \in L$. São equivalentes:

a) $H x \cap H y \neq \emptyset$ implica que $H x=H y$;

b) $H(h x)=H x$ para todo $h \in H$.

Demonstração. Vamos supor que valha o item b e sejam $h_{1} x=h_{2} y \in H x \cap H y$. Então segue que $H x=H\left(h_{1} x\right)=H\left(h_{2} y\right)=H y$. Reciprocamente, para todo $h \in H$, $h x \in H(h x) \cap H x \operatorname{logo} H(h x)=H x$.

Definição 1.1.6. Sejam a,b.c $\in$ L. O comutador de a e b é o único elemento $(a, b) \in L$ tal que $a b=b a(a, b)$. O associador de $a, b$ e c é o único elemento $(a, b, c) \in L$ tal que $(a b) c=[a(b c)](a, b, c)$.

Definição 1.1.7. Seja L um loop. Os conjuntos

$$
\mathcal{N}_{\lambda}(L)=\{a \in L:(a, x, y)=1 \quad \text { para todos } \quad x, y \in L\}
$$




$$
\mathcal{N}_{\rho}(L)=\{a \in L:(x, y, a)=1 \quad \text { para todos } \quad x, y \in L\}
$$

e

$$
\mathcal{N}_{\mu}(L)=\{a \in L:(x, a, y)=1 \quad \text { para todos } \quad x, y \in L\}
$$

são cahamdos, respectivamente, núcleo à esquerda, núcleo à direita e núcleo intermediário de L.

$O$ núcleo de $L$ é o conjunto $\mathcal{N}(L)=\mathcal{N}_{\lambda}(L) \cap \mathcal{N}_{\mu}(L) \cap \mathcal{N}_{\rho}(L)$ e o centro de $L$ é o conjunto

$$
\mathcal{Z}(L)=\{a \in \mathcal{N}(L):(a, x)=1 \quad \text { para todo } \quad x \in L\}
$$

Proposição 1.1.8. Cada um dos núcleos de um loop é um subloop associativo e, portanto, um grupo. Em particular, o centro de um loop é um grupo abeliano.

Demonstração. Seja $L$ um loop. Desde que $1 \in \mathcal{N}_{\mu}(L)$ temos $\mathcal{N}_{\mu}(L) \neq \emptyset$. Para quaisquer $a, b \in \mathcal{N}_{\mu}(L)$ e $x, y \in$ Ltemos

$$
[x(a b)] y=[(x a) b] y=(x a)(b y)=x[a(b y)]=x[(a b) y]
$$

Portanto ab $\in \mathcal{N}_{\mu}(L)$. Além disso $\left(a^{\rho} a\right) a^{\rho}=a^{\rho}\left(a a^{\rho}\right)=a^{\rho} 1=1 a^{\rho}$ e, pela lei do cancelamento, temos $a^{\rho} a=1$ que implica $a^{\lambda}=a^{\rho}$. Logo, qualquer $a \in \mathcal{N}_{\mu}(L)$ possui inverso bilateral, denotado por $a^{-1}$. Mais ainda, se $a \in \mathcal{N}_{\mu}(L)$ então $R_{a}^{-1}=R_{a^{-1}}$, pois $(x a) a^{-1}=x\left(a a^{-1}\right)=x$ qualquer que seja $x \in L$ e, da mesma forma, $L_{a}^{-1}=L_{a^{-1}}$. Para quaisquer $x, y \in L$, podemos escrever $x=$ ta para algum $t \in L$ e então temos:

$$
\left(x a^{-1}\right) y=\left[(t a) a^{-1}\right] y=\left[t\left(a a^{-1}\right)\right] y=t y
$$

e

$$
x\left(a^{-1} y\right)=(t a)\left(a^{-1} y\right)=t\left[a\left(a^{-1} y\right)\right]=t\left[L_{a} L_{a^{-1}}(y)\right]=t y .
$$

Ou seja, $a^{-1} \in \mathcal{N}_{\mu}(L)$. Como $a(b c)=(a b) c$, para quaisquer que sejam $a, b, c \in \mathcal{N}_{\mu}(L)$, obetmos que $\mathcal{N}_{\mu}(L)$ é um grupo. 
Consideremos agora, $\mathcal{N}_{\lambda}(L)$. É fácil ver que $1 \in \mathcal{N}_{\lambda}(L)$ e que ab $\in \mathcal{N}_{\lambda}(L)$ quaisquer que sejam $a, b \in \mathcal{N}_{\lambda}(L)$. Para $a \in \mathcal{N}_{\lambda}(L)$, temos $\left(a a^{\lambda}\right) a=a\left(a^{\lambda} a\right)=a 1=1 a$ e, portanto a possui um inverso bilateral em $L$, que será denotado por $a^{-1}$. Além disso, qualquer que seja $x \in L, a\left(a^{-1} x\right)=\left(a a^{-1}\right) x=1 x=x$, o que implica que $L_{a}^{-1}=L_{a^{-1}}$. Tome $x, y \in L$, então

$$
a\left[\left(a^{-1} x\right) y\right]=\left[a\left(a^{-1} x\right)\right] y=L_{a} L_{a^{-1}}(x) y=x y
$$

$e$

$$
a\left[a^{-1}(x y)\right]=\left(a a^{-1}\right)(x y)=x y \text {. }
$$

Assim, pela lei do cancelamento, obtemos $\left(a^{-1} x\right) y=a^{-1}(x y)$ para quaisquer $x, y \in L$, ou seja, $a^{-1} \in \mathcal{N}_{\lambda}(L)$ e, portanto, este é um subloop de L. Como $(a b) c=a(b c)$ para todos $a, b, c \in \mathcal{N}_{\lambda}(L)$, temos que este é um grupo.

Para $\mathcal{N}_{\rho}(L)$, observemos que, se $(L,$.$) é um loop, fazendo x * y=y x$, temos um loop $(L, *)$ que será chamado de loop oposto a $(L,$.$) e denotado por L^{o p}$. Além disso $\mathcal{N}_{\rho}(L)=\mathcal{N}_{\lambda}\left(L^{o p}\right)$. Logo $\mathcal{N}_{\rho}(L)$ é um grupo.

Por definição, $\mathcal{N}(L)=\mathcal{N}_{\mu}(L) \cap \mathcal{N}_{\lambda}(L) \cap \mathcal{N}_{\rho}(L)$, donde segue, facilmente, que $\mathcal{N}(L)$ é um subgrupo de $L$.

Finalmente, para $\mathcal{Z}(L)$, basta observarmos que se $a, b \in \mathcal{Z}(L) \subset \mathcal{N}(L)$ então ab $\in$ $\mathcal{Z}(L)$. De fato,

$$
(a b) x=a(b x)=a(x b)=(a x) b=(x a) b=x(a b),
$$

o que completa a demonstração.

Temos que a condição b) da proposição 1.1.5 vale se o subloop $H$ for um dos núcleos ou o centro do loop $L$. Portanto se $L$ for um loop finito, então os números $\left|\mathcal{N}_{\lambda}(L)\right|,\left|\mathcal{N}_{\mu}(L)\right|,\left|\mathcal{N}_{\rho}(L)\right|,|\mathcal{N}(L)|$, e $|\mathcal{Z}(L)|$ dividem $|L|$.

Definição 1.1.9. Seja $L$ um loop. Um subloop $H$ de $L$ é dito normal se para quaisquer $x, y \in L$ temos

$$
H x=x H, \quad H(x y)=(H x) y, \quad(x H) y=x(H y), \quad e \quad(y x) H=y(x H) .
$$


Usaremos a notação $H \triangleleft L$ para indicar que o subloop $H$ é normal em $L$.

Sejam $L$ um loop e $H \triangleleft L$ e consideremos o conjunto $L / H=\{x H: x \in L\}$. Vamos definir a seguinte operação binária em $L / H$ : para $x H, y H \in L / H$,

$$
(x H)(y H)=(x y) H .
$$

Notemos que se $x H=x^{\prime} H$ e $y H=y^{\prime} H$ então

$$
\begin{gathered}
(x y) H=H(x y)=(H x) y=(x H) y=\left(x^{\prime} H\right) y= \\
=x^{\prime}(H y)=x^{\prime}(y H)=x^{\prime}\left(y^{\prime} H\right)=x^{\prime}\left(H y^{\prime}\right)= \\
=\left(x^{\prime} H\right) y^{\prime}=\left(H x^{\prime}\right) y^{\prime}=H\left(x^{\prime} y^{\prime}\right)=\left(x^{\prime} y^{\prime}\right) H .
\end{gathered}
$$

Ou seja, a operação definida acima está bem definida. É de fácil verificação que esta operação dá a $L / H$ uma estrutura de loop cujo elemento neutro é $1 H=H$.

Definição 1.1.10. O loop $L / H$ é chamado de loop quociente de $L$ por $H$.

Se $H \triangleleft L$, então temos $H(h x)=(H h) x=H x$ para todo $x \in L$ e todo $h \in H$. Assim, as distintas classes laterais de $H$ em $L$ são disjuntas. Então, se $L$ for um loop finito, teremos que $|H|$ divide $|L|$ e $|L / H|=\frac{|L|}{|H|}$.

Definição 1.1.11. Sejam $L, L^{\prime}$ loops e uma aplicação $f:(L,.) \longrightarrow\left(L^{\prime}, *\right)$. Dizemos que $f$ é um homomorfismo se $f(x . y)=f(x) * f(y)$ quaisquer que sejam $x, y \in L$. Um isomorfismo entre os loops $L, L^{\prime}$ é um homomorfismo bijetivo. Um automorfismo do loop $L$ é um isomorfismo de $L$ em $L$.

Observação. As oprerações de $L$ e $L^{\prime}$ terão a mesma notação embora elas sejam, eventualmente, diferentes.

Definição 1.1.12. O kernel de um homomorfismo de loops $f: L \longrightarrow L^{\prime}$ é o conjunto

$$
\operatorname{ker}(f)=\{x \in L: f(x)=1\}
$$

e a imagem de $f$ é o conjunto

$$
f(L)=\left\{y=f(x) \in L^{\prime}: x \in L\right\} .
$$


Lema 1.1.13. Se $f: L \longrightarrow L^{\prime}$ for um homomorfismo de loops, então ker $(f)$ é um subloop de $L$.

Demonstração. Como $f(1)=(1.1)=f(1) f(1)$ temos que $f(1)=1$, e portanto, $K=\operatorname{ker}(f) \neq \emptyset$. Sejam $x, y, z \in L$ com $x y=z$. Então

- Se $x, y \in K$ vale $f(z)=f(x y)=f(x) f(y)=1$, ou seja $z \in K$;

- Se $y, z \in K$ vale $f(x)=f(x) f(y)=f(x y)=f(z)=1$, ou seja $x \in K$;

- Se $x, z \in K$ vale $f(y)=f(x) f(y)=f(x y)=f(z)=1$, ou seja $y \in K$.

Segue, portanto, do item c) da proposição 1.1 .3 que $K$ é um suloop de $L$.

Lema 1.1.14. Se $f: L \longrightarrow L^{\prime}$ for um homomorfismo de loops, então $K=k e r(f)$ é um subloop normal em $L$.

Demonstração. No lema anterior mostramos que $K$ é um subloop de L. Sejam $x, y \in L$.

- Vamos mostrar que $K x=x K$.

Seja $x k \in x K$ um elemento arbitrário. Existe um único elemento $a \in L$ que satifaz a equação ax $=x k$ e nosso objetivo é mostrar que a $\in K$. De fato temos que $f(a) f(x)=f(a x)=f(x k)=f(x) f(k)=f(x)$. Logo, aplicando a lei do cancelamento, temos $f(a)=1$, como desejado. Analogamente se $a \in L$ satisfaz a equação $x a=k x$, para um lemento $k \in K$ arbitrário, vamos ter, necessariamente, $a \in K$. Portanto $K x=x K$.

- Considere $d=k(x y) \in K(x y)$ e a equação $(z x) y=d$. Existe um único $a \in L$ tal que $(a x) y=d=k(x y)$. Quermos mostrar que $a \in K$. Temos $f(x y)=f(k) f(x y)=f(k(x y))=f((a x) y)=f(a x) f(y)$, e assim, $f(x) f(y)=$ $(f(a) f(x)) f(y)$. Então $f(a) f(x)=f(x)$, isto é, $f(a)=1$.

Da mesma forma considerando $d=(k x) y \in(K x) y$ e a equação $z(x y)=d$ temos que existe um único $a \in L$ tal que $a(x y)=(k x) y$ e assim $f(a) f(x y)=$ 
$f(a(x y))=f((k x) y)=(f(k) f(x)) f(y)=f(x) f(y)=f(x y)$ e então $f(a)=1$. $\operatorname{Logo}(K x) y=K(x y)$.

- A prova de que $(x K) y=x(K y)$ é feita de maneira inteiramenta análoga à prova de que $(K x) y=K(x y)$.

- Finalmente $(y x) K=K(y x)=(K y) x=(y K) x=y(K x)=y(x K)$.

Logo K é um subloop normal de L.

Lema 1.1.15. Um subloop $H \leq L$ é normal em $L$ se e somente se for kernel de um homomorfismo com domínio $L$.

Demonstração. No lema anterior, vimos que se $K=K$ er $(f)$ para algum homomorfismo de loops $f: L \longrightarrow L^{\prime}$ então $K$ é um subloop normal de L. Reciprocamente se $K$ é um subloop normal de $L$ então a aplicação $\pi: L \longrightarrow L / K$ dada por $\pi(a)=a K$ é um homomorfismo de loops com $\operatorname{ker}(\pi)=K$.

Definição 1.1.16. O conjunto

$$
\mathcal{I}(L)=\{f \in \mathcal{M}(L): f(1)=1\}
$$

é chamado grupo das aplicações internas de L.

Claramente $\mathcal{I}(L)$ é um subgrupo de $\mathcal{M}(L)$. Exibiremos uma caracterização bastante útil para o grupo $\mathcal{I}(L)$.

Proposição 1.1.17. O grupo das aplicações internas $\mathcal{I}(L)$ é gerado pelas aplicações da forma

$$
\begin{gathered}
T_{(x)}=L_{x}^{-1} R_{x}, \\
R_{(x, y)}=R_{x y}^{-1} R_{y} R_{x}
\end{gathered}
$$

e

$$
L_{(x, y)}=L_{y x}^{-1} L_{y} L_{x}
$$

para $x, y \in L$. 
Demonstração. Considere $F=\left\{R_{(x, y)}, L_{(x, y)}, T_{x} ; x, y \in L\right\} \subset \mathcal{M}(L)$. Queremos mostrar que $\mathcal{I}(L)=\langle F\rangle$.

Considere o conjunto $K=\left\{g \in \mathcal{M}(L) ; g \in R_{g(1)}\langle F\rangle\right\}$, onde $R_{g(1)}\langle F\rangle$ denota a classe lateral do subgrupo $\langle F\rangle$ em $\mathcal{M}(L)$. Se $g(1)=t$, para cada $x \in L$ temos

$$
R_{x}(g(1))=R_{x}(t)=t x .
$$

Para cada $g \in K$ existe $\phi \in\langle F\rangle$ tal que

$$
g=R_{g(1)} \phi=R_{t} \phi
$$

Assim temos

$$
R_{x} g=R_{x} R_{t} \phi=R_{t x} R_{(t, x)} \phi=R_{R_{x}(g(1))} R_{(t, x)} \phi .
$$

Ou seja $R_{x} g \in K$.

Temos que, se

$$
L_{x}(g(1))=L_{x}(t)=x t,
$$

então

$$
L_{x} g=L_{x} R_{t} \phi=R_{L_{x}(g(1))} T_{x t}^{-1} L_{(t, x)} T_{t} \phi,
$$

isto é $L_{x} g \in K$. Do memso modo temos que $L_{x}^{-1} g \in K$ e $R_{x}^{-1} g \in K$. Assim $\mathcal{M}(L) K \subset K \subset \mathcal{M}(L) \subset \mathcal{M}(L) K$. Em outras palavras, para toda aplicção $f \in$ $\mathcal{M}(L), f \in R_{f(1)}\langle F\rangle$. Se $f \in \mathcal{I}(L)$ temos $f(1)=1$ e assim $f \in\langle F\rangle$.

Reciprocamnte, vamos mostrar que para quaisquer $x, y \in L$ temmos

$$
\begin{gathered}
R_{(x, y)}(1)=R_{x y}^{-1} R_{y} R_{x}(1)=R_{x y}^{-1}(x y)=1, \\
L_{(x, y)}(1)=L_{y x}^{-1} L_{y} L_{x}(1)=L_{y x}^{-1}(y x)=1
\end{gathered}
$$

$e$

$$
T_{x}(1)=L_{x}^{-1} R_{x}(1)=L_{x}^{-1}(x)=1 .
$$

Então $\mathcal{I}(L)=\langle F\rangle$. 
Lema 1.1.18. Um subloop $H \leq L$ é normal em $L$ se e somente se $f(H) \subset H$, para qualquer $f \in \mathcal{I}(L)$.

Demonstração. Sejam $x, y \in L$ e $h \in H$. Como $R_{(x, y)}(h)=R_{x y}^{-1} R_{y} R_{x}(h)=$ $R_{x y^{-1}}((h x) y)$ temos que $R_{(x, y)}(h) \in H$ se e somente se $(H x) y=H(x y)$. Da mesma forma temos que $L_{(x, y)}(h) \in H$ se e somente se $y(x H)=(x y) H$ e $T_{x}(h) \in H$ se e somente se $x H=H x$. Para completar, a demosntração basta observar que se para um subloop $H$ de $L$ valerem as propriedades $(H x) y=H(x y), y(x H)=(x y) H$ e $x H=H x$ para quaisquer $x, y \in L$ temos

$$
(x H) y=(H x) y=H(x y)=(x y) H=x(y H)=x(H y)
$$

para quaisquer $x, y \in L$.

Para um automorfismo artrário $S$ de $L$, é fácil ver que, para quaisquer $x, y \in L$,

$$
\begin{array}{ccc}
S R_{x} S^{-1}=R_{S(x)} & \text { e } & S L_{x} S^{-1}=L_{S(x)} \\
S R_{(x, y)} S^{-1}=R_{(S(x), S(y))} & \text { e } & S L_{(x, y)} S^{-1}=L_{(S(x), S(y))} \\
S T_{x} S^{-1}= & T_{S(x)}
\end{array}
$$

\subsection{Loops com a Propriedade do Inverso}

Definição 1.2.1. Um loop $L$ é dito ser um loop com a propriedade do inverso se para cada $x \in L$ existe um inverso bilateral $x^{-1}$, isto é $x x^{-1}=x^{-1} x=1$, e ainda

$$
x^{-1}(x y)=y
$$

e

$$
(y x) x^{-1}=y
$$

para todos $x, y \in L$. 
Para simplificar a liguagem, chamaremos um loop com a proriedade do inverso $L$ de $P I$-loop. Para um $P I$-loop $L$ definimos $J: L \longrightarrow L$ por $J(x)=x^{-1}$.

Proposição 1.2.2. Sejam $L$ um PI-loop e $I: L \longrightarrow L$ a aplicação identidade. Então:

1. $J^{2}=I$;

2. $R_{x}^{-1}=R_{x^{-1}}$ e $L_{x}^{-1}=L_{x^{-1}}$ para todo $x \in L$;

3. $(x y)^{-1}=y^{-1} x^{-1}$ para todos $x, y \in L$;

4. $J L_{x} J=R_{x^{-1}}$ e $J R_{x} J=L_{x^{-1}}$.

\section{Demonstração.}

1. De $x x^{-1}=x^{-1} x=1$, para qualquer que seja $x$ no PI-loop L, temos que $\left(x^{-1}\right)^{-1}=x$ donde segue $J^{2}=I$.

2. Temos que $(y x) x^{-1}=y$ para todos $x, y \in L$ se e somente se $R_{x^{-1}} R_{x}=I$ para todo $x \in L$. Da definição de PI-loop e do item 1, segue que $R_{x} R_{x^{-1}}=I$ pois $\left(y x^{-1}\right) x=\left(y x^{-1}\right)\left(x^{-1}\right)^{-1}$. Logo $R_{x}^{-1}=R_{x^{-1}}$. Da modo análogo, obtemos $L_{x}^{-1}=L_{x^{-1}}$ para todo $x \in L$.

3. Basta notar que se $x y=z$ então $x=(x y) y^{-1}=z y^{-1}$ e, assim, $y^{-1}=$ $z^{-1}\left(z y^{-1}\right)=z^{-1} x$. Logo $z^{-1}=\left(z^{-1} x\right) x^{-1}=y^{-1} x^{-1}$.

4. Para $x, y \in L$ temos $J L_{x} J(y)=J L_{x}\left(y^{-1}\right)=J\left(x y^{-1}\right)=y x^{-1}=R_{x^{-1}}(y) e$ $J R_{x} J(y)=J R_{x}\left(y^{-1}\right)=J\left(y^{-1} x\right)=x^{-1} y=L_{x^{-1}}(y)$, o que completa a demonstração.

Corolário 1.2.3. Um subconjunto não vazio $H$ de um PI-loop L é um subloop se e somente se $x y, x^{-1} \in H$ para quaisquer $x, y \in H$. 
Proposição 1.2.4. Se L for um PI-loop então $\mathcal{N}_{\lambda}(L)=\mathcal{N} \mu(L)=\mathcal{N}_{\rho}(L)$. Consequentemente, todos os núcleos de um PI-loop são iguais.

Demonstração. Seja $x \in \mathcal{N}_{\lambda}(L)$. Então $x^{-1} \in \mathcal{N}_{\lambda}(L)$, pois este é um grupo. Assim para $a, b \in L$ temos que $\left(x^{-1} a^{-1}\right) b^{-1}=x^{-1}\left(a^{-1} b^{-1}\right)$ e tomando o iverso nessa igualdade temos que $b(a x)=(b a) x$, isto é, $x \in \mathcal{N}_{\rho}(L)$. Portanto $\mathcal{N}_{\lambda}(L) \subset \mathcal{N}_{\rho}(L)$. Da mesma maneira, se $x \in \mathcal{N}_{\rho}(L)$ então $\left(a^{-1} b^{-1}\right) x^{-1}=a^{-1}\left(b^{-1} x^{-1}\right)$ o que implica que $x(b a)=(x b)$ a para quaisquer $a, b \in L$. Logo, $\mathcal{N}_{\rho}(L) \subset \mathcal{N}_{\lambda}(L)$ e assim $\mathcal{N}_{\rho}(L)=\mathcal{N}_{\lambda}(L)$.

Para $x \in \mathcal{N}_{\mu}(L)$, temos $(a x) b=a(x b)$ para todos $a, b \in L$, isto é

$$
b=(a x)^{-1}[a(x b)]=\left(x^{-1} a^{-1}\right)[a(x b)]
$$

- Para quaisquer $a, c \in L$ existe $b \in L$ tal que $c=a(x b)$ e então $b=\left(x^{-1} a^{-1}\right) c$. Por outro lado, $x^{-1}\left(a^{-1} c\right)=x^{-1}(x b)=b$. Portanto

$$
\left(x^{-1} a^{-1}\right) c=x^{-1}\left(a^{-1} c\right)
$$

ou seja, $x^{-1} \in \mathcal{N}_{\lambda}(L)$, e com isso $x \in \mathcal{N}_{\lambda}(L)$. Logo $\mathcal{N}_{\mu}(L) \subset \mathcal{N}_{\lambda}(L)$. Reciprocamente, se $x \in \mathcal{N}_{\lambda}(L),(x a) b=x(a b)$ para todos $a, b \in L$. Assim

$$
b=\left(a^{-1} x^{-1}\right)[x(a b)]
$$

Para $a, c \in L$, existe $b \in L$ tal que $x(a b)=c$ e assim $b=\left(a^{-1} x^{-1}\right) c$. Também temos $a^{-1}\left(x^{-1} c\right)=a^{-1}(a b)=b$. Assim

$$
a^{-1}\left(x^{-1} c\right)=\left(a^{-1} x^{-1}\right) c
$$

então $x^{-1} \in \mathcal{N}_{\mu}(L)$ e, então $x \in \mathcal{N}_{\mu}(L)$. Logo $\mathcal{N}_{\lambda}(L) \subset \mathcal{N}_{\mu}(L)$ e assim, $\mathcal{N}_{\lambda}(L)=$ $\mathcal{N}_{\mu}(L)$.

Definição 1.2.5. O índice do subloop $H$ em $L$ é o número minimal de classes laterais à direita de $H$ cuja reunião é igual a $L$. 
Vamos denotar o índice de $H$ em $L$ por $[L: H]$.

Suponhamos $L$ um $P I$-loop e $H \subset L$ um subloop. Desde que $\left\{h^{-1}: h \in H\right\}=$ $H$ temos que $H x \mapsto x^{-1} H$ é uma bijeção entre a família de classes laterais à direita de $H$ e a família de classes laterais à esquerda de $H$. Então, o índice de $H$ em $L$ é o número minimal de classes laterais à esquerda de $H$ cuja reunião é igual a $L$. Além disso se $L$ for finito e puder ser escrito como reunião disjunta de classes laterais de $H$ teremos $[L: H]=\frac{|L|}{|H|}$.

\section{$1.3 \quad$ Exemplos de Loops}

No início deste capítulo, mencionamos que um loop associoativo é um grupo. Daremos agora dois exemplos de loops que não são associativos.

Exemplo 1.3.1. Considere o conjunto $S=\{1,2,3,4,5\} \subset \mathbb{N}$, munido da operação binária descrita na tabela abaixo.

\begin{tabular}{|l|l|l|l|l|l|}
\hline$*$ & $\mathbf{1}$ & $\mathbf{2}$ & $\mathbf{3}$ & $\mathbf{4}$ & $\mathbf{5}$ \\
\hline $\mathbf{1}$ & 1 & 2 & 3 & 4 & 5 \\
\hline 2 & 2 & 1 & 4 & 5 & 3 \\
\hline 3 & 3 & 5 & 1 & 2 & 4 \\
\hline 4 & 4 & 3 & 5 & 1 & 2 \\
\hline 5 & 5 & 4 & 2 & 3 & 1 \\
\hline
\end{tabular}

Como cada elemento de $S$ aparece uma vez, e somente uma vez, temos que $(S, *)$ é um quase-grupo. Além disso, 1 é o elemento neutro de $S$. Entretanto $3 *(3 * 4)=$ $3 * 2=5$ e $(3 * 3) * 4=1 * 4$, portanto $(S, *)$ não é associativo.

Exemplo 1.3.2. Sejam $G$ um grupo não abeliano, u uma indetreminada e considere o conjunto formal $G u=\{g u: g \in G\}$. Vamos definir $L=G \cup G u$ onde esta reunião é disjunta e vamos estender a operação de $G$ a $L$ da seguinte maneira:

$$
g(h u)=(h g) u,
$$




$$
(g u) h=\left(g h^{-1}\right) u
$$

e

$$
(g u)(h u)=h^{-1} g
$$

para quaisquer $g, h \in G$.

Afirmamos que $L$ é um loop. De fato, seja a equação $a x=b \operatorname{com} a, b \in L$.

- Se $a, b \in G$ então a equação tem única solução em $G \subset L$;

- Se $a=g_{1} u \in G u$ e $b \in G$, façamos $x=g_{1} b^{-1} u$ e, assim $\left(g_{1} u\right)\left(g_{1} b^{-1} u\right)=$ $\left(g_{1} b^{-1}\right)^{-1} g_{1}=b$. Portanto $x=g_{1} b^{-1} u \in G u \subset L$ é uma solução de $a x=b$. Se y for uma outra solução de $a x=b, y$ deve pertencer $a G u$, pois $b \in G e$ $a \in G u$. Assim $y=g_{2} u$ com $g_{2} \in G$ e $b=\left(g_{1} u\right)\left(g_{2} u\right)=g_{2}^{-1} g_{1}$, o que implica que, $g_{2}=g_{1} b^{-1}$ e, logo, a solução é única.

- Supondo $a \in G, b=h_{1} u \in G u$ e fazendo $x=\left(h_{1} a^{-1}\right) u \in G u$ temos que ax $=$ $a\left(h_{1} a^{-1} u\right)=h_{1} a^{-1} a u=b$ e, assim, a equação tem solução. Se $y=h_{2} u \in G u$ for outra solução de ax $=b$ (qualquer solucão de ax $=b$ está em Gu pois a $\in G$ e $b \in G)$ temos $h_{1} u=a\left(h_{2} u\right)=h_{2} a u$, ou seja, $h_{2}=h_{1} a^{-1}$ e a solução é única.

- Agora, se $a=a_{1} x, b=b_{1} u \in G u$ onde $a_{1}, b_{1} \in G$, faça $x=b_{1}^{-1} a_{1} \in G$ e então $\left(a_{1} u\right)\left(b_{1}^{-1} a_{1}\right)=a_{1}\left(b_{1}^{-1} a_{1}\right)^{-1} u=b_{1} u=b$, o que implica que a equação $a x=b$ tem solução. E a solução é unica pois se $g \in G$ for outra solução teremos $b_{1} u=\left(a_{1} u\right) g=\left(a_{1} g^{-1}\right) u$ e assim $g=b_{1}^{-1} a_{1}$.

De maneira inteiremente análoga, prova-se que a equação xa = b tem única solução em $L$, quaisquer que sejam $a, b \in L$. Também é de fácil verificação que ea $=a e=a$ para todo $a \in L$, onde e é o elemento neutro de $G$. Com isso temos que L é um loop. Verifiquemos agora que $L$ não é um grupo. Para isso, tomemos gu, hu,lu $\in G u$. Temos que

$$
(g u)((h u)(l u))=(g u)\left(l^{-1} h\right)=\left(g h^{-1} l\right) u
$$


$e$

$$
((g u)(h u))(l u)=\left(h^{-1} g\right)(l u)=\left(l h^{-1} g\right) u .
$$

Assim $(g u)((h u)(l u))=((g u)(h u))(l u)$ se, e somente se,

$$
g h^{-1} l=l h^{-1} g
$$

Tomando $h=e, g \in G \backslash Z(G)$ e $l \in G \backslash C_{G}(g)$, temos que a igualdade 1.4 não se verifica e, portanto, $L$ não é associativo. Logo L não é um grupo. 


\section{Capítulo 2}

\section{A-loops}

Neste capítulo estudaremos um importante tipo de loop, loops cujas aplicações internas são automorfismos.

\subsection{Propriedades Gerais}

Definição 2.1.1. Um loop $L$ é chamado $\boldsymbol{A}$-loop se cada elemento $f \in \mathcal{I}(L)$ for um automorfismo de L.

Definição 2.1.2. Um subloop $H \leq L$ é um subloop característico de $L$ se $f(H) \subset$ $H$, para todo automorfismo $f$ de $L$.

Teorema 2.1.3. Seja L um A-loop. Então:

1. Todo subloop de L é um A-loop;

2. Todo subloop característico de L é um subloop normal de $L$;

3. Os núcleos e o centro de L são subloops normais de $L$;

4. Se $H \triangleleft L$, então $L / H$ é um A-loop.

Demonstração. 
1. Seja $H \leq L$. Temos que $\mathcal{I}(H)=\left\langle R_{(x, y)}, L_{(x, y)}, T_{(x)} \mid x, y \in H\right\rangle$. Como L é um A-loop temos que $\mathcal{I}(H) \subset \mathcal{I}(L) \subset$ Aut $(L)$ e, por isso, $\left.R_{(x, y)}\right|_{H},\left.L_{(x, y)}\right|_{H},\left.T_{(x)}\right|_{H}$ são homomorfismos injetivos de $H$ para quaisquer $x, y \in H$. Para $b \in H$, existe $a \in L$ tal que $R_{(x, y)}(a)=R_{x y}^{-1} R_{y} R_{x}(a)=R_{x y}^{-1}(a x . y)=b$, ou seja, $b(x y)=(a x) y$. Então ax $\in L$ é a única solução da equação $z y=b(x y)$ e, como b, $x$ e y são elementos do loop $H$, podemos concluir que ax $=h \in H$. Assim a é a única solução de $z x=h$ e, pelo mesmo argumento, concluimos que $a \in H$. Portanto $R_{(x, y)}: H \longrightarrow H$ é um automorfismo de $H$, para todos $x, y \in H$. Analogamente, concluímos que $L_{(x, y)}, T_{(x)} \in$ Aut $(H)$ para todos $x, y \in H$. Logo, H é um A-loop.

2. Se $H \leq L$ é um subloop característico de $L$, temos, por definição, que $f(H) \subset H$ para toda $f \in \operatorname{Aut}(L)$. Assim $f(H) \subset H$ para qualquer $f \in \mathcal{I}(L) \subset \operatorname{Aut}(L)$ e, portanto, $H$ é um subloop normal de $L$.

3. Sejam $f \in \operatorname{Aut}(L)$ e $x, y \in L$. Então existem $u, v \in L$ tais que $f(u)=x$ e $f(v)=y$. Se $a \in \mathcal{N}_{\lambda}(L)$, temos $\left(a f^{-1}(u)\right) f^{-1}(v)=a\left(f^{-1}(u) f^{-1}(v)\right)$ e assim $f\left(\left(a f^{-1}(u)\right) f^{-1}(v)\right)=f\left(a\left(f^{-1}(u) f^{-1}(v)\right)\right)$ ou, equivalentemente, $f(a) x . y=$ $f(a) . x y$. Portanto $f(a) \in \mathcal{N}_{\lambda}(L)$, e então, $\mathcal{N}_{\lambda}(L)$ é um subloop característico de L. Logo $\mathcal{N}_{\lambda}(L)$ é um subloop normal de L. De maneira análoga temos que $\mathcal{N}_{\mu}(L)$ e $\mathcal{N}_{\rho}(L)$ são subloops normais de $L$.

Se $a \in \mathcal{N}(L)=\mathcal{N}_{\lambda}(L) \cap \mathcal{N}_{\mu}(L) \cap \mathcal{N}_{\rho}(L)$ então $f(a) \in \mathcal{N}_{\lambda}(L) \cap \mathcal{N}_{\mu}(L) \cap \mathcal{N}_{\rho}(L)$. Então $\mathcal{N}(L)$ é um subloop característico de $L$ e, portanto, um subloop normal de $L$.

Para $a \in \mathcal{Z}(L) \subset \mathcal{N}(L)$ temos que $f(a) \in \mathcal{N}(L)$ e, além disso, como $f^{-1}(u) a=$ $a f^{-1}(u)$ temos $f(a) x=x f(a)$, o que implica que $f(a) \in \mathcal{Z}(L)$. Logo $\mathcal{Z}(L)$ é um subloop característico e, portamto, normal de $L$.

4. Para cada $f \in \mathcal{I}(L)$, defina $f^{\prime}: L / H \longrightarrow L / H$ onde $f^{\prime}(x H)=f(x) H$. Sabemos $x H=y H$ se e somente se para todo $h \in H$ existem $h_{1}, h_{2} \in H$ tais que $x h=y h_{1}$ e $y h=x h_{2}$. Como $H \triangleleft L$ e como $f$ é um automorfismo de L, temos que

$$
f(x) f(h)=f(x h)=f\left(y h_{1}\right)=f(y) f\left(h_{1}\right)
$$


e

$$
f(y) f(h)=f(y h)=f\left(x h_{2}\right)=f(x) f\left(h_{2}\right)
$$

onde $f(h), f\left(h_{1}\right), f\left(h_{2}\right) \in H$. Assim teremos $f(x) H=f(y) H$ e então $f^{\prime}$ está bem definida.

Além disso $f^{\prime}(H)=f^{\prime}(1 H)=f(1) H=1 H=H$, isto é $f^{\prime} \in \mathcal{I}(L / H)$. Nosso objetivo é mostrar que a aplicação $\tau: \mathcal{I}(L) \longrightarrow \mathcal{I}(L / H)$, definido por $\tau(f)=f^{\prime}$ é um homomorfismo sobrejetor de grupos.Para $f, g \in \mathcal{I}(L)$ e $x \in L$ temos

$$
(f g)^{\prime}(x H)=f(g(x)) H=f^{\prime}(g(x) H)=f^{\prime}\left(g^{\prime}(x H)\right),
$$

ou seja $(f g)^{\prime}=f^{\prime} g^{\prime}$ e assim $\tau$ é um homomorfismo de grupos.

Para $x, y \in L$ considere $R_{(x H, y H)}, L_{(x H, y H)}$ e $T_{x H}$. Seja $z \in L$,

$$
R_{(x H, y H)}(z H)=R_{(y x) H}^{-1}(((z x) y) H)=w H
$$

onde $(w(x y)) H=((z x) y) H$. Por outro lado,

$$
R_{(x, y)}^{\prime}(z H)=\left(R_{(x, y)}(z)\right) H=w H
$$

onde $w(x y)=(z x) y$. Portanto $R_{(x, y)}^{\prime}=R_{(x H, y H)}$. Da memsa forma, $L_{(x, y)}^{\prime}=$ $L_{(x H, y H)} T_{x}^{\prime}=T_{x H}$. Assim $\tau$ é um epimorfismo de grupos.

Finalmente, para cada $f^{\prime} \in \mathcal{I}(L / H)$, onde $f \in \mathcal{I}(L)$, e para quaisquer $x, y \in L$ vale

$f^{\prime}(x H y H)=f^{\prime}(x y H)=f(x y) H=(f(x) f(y)) H=(f(x) H)(f(y) H)=f^{\prime}(x H) f^{\prime}(y H)$.

Logo $f^{\prime}$ é um automorfismo de $L / H$ e, portanto, $L / H$ é um A-loop.

Um subconjunto associativo (comutativo) $K \subset L$ é chamado subconjunto associativo (comutativo) maximal se $K$ não estiver contido propriamente em nenhum subconjunto associativo (comutativo) de $L$. Pelo Lema de Zorn temos que qualquer subconjunto associativo (comutativo) de $L$ está contido em,pelo menos, um subconjunto associativo (comutativo) maximal de $L$. 
Lema 2.1.4. Sejam $L$ um loop, $S$ um conjunto não vazio de automorfismos de $L$ e $H$ o subconjunto dos elementos de $L$ que são fixos por todos os elementos de $S$, isto é, $H=\{x \in L: f(x)=x$ para todo $f \in S\}$. Então $H$ é um subloop de $L$.

Demonstração. É fácil ver que $H \neq \emptyset$ pois $1 \in H$. Sejam $x, y, z \in L$ com $x y=z$.

- Se $x, y \in H$, temos $f(z)=f(x y)=f(x) f(y)=x y=z, \log o z \in H$.

- Se $x, z \in H$, temos $z=f(z)=f(x y)=f(x) f(y)=x f(y), \operatorname{logo} f(y)=y e$ $y \in H$.

- Se $y, z \in H$, temos $z=f(z)=f(x y)=f(x) f(y)=f(x) y, \log o f(x)=x e$ $x \in H$.

Concluímos, então, que Hé um subloop de $L$.

Observação. Notemos que para $x \in H$ temos $1=f(1)=f\left(x x^{\rho}\right)=f(x) f\left(x^{\rho}\right)=$ $x f\left(x^{\rho}\right)$ e $1=f(1)=f\left(x^{\lambda} x\right)=f\left(x^{\lambda}\right) f(x)=f\left(x^{\lambda}\right) x$ para todo $f \in S$. Portanto $f\left(x^{\rho}\right)=x^{\rho}$ e $f\left(x^{\lambda}\right)=x^{\lambda}$ para todo $f \in S$. Logo $x^{\lambda}, x^{\rho} \in H$.

Teorema 2.1.5. Seja L um A-loop. Então

1. Todo subconjunto comutativo maximal, $C$, é um subloop de $L$.

2. Todo subconjunto associativo maximal, A, é um subloop de L.

3. L comuta potências, isto é, $x^{m} x^{n}=x^{n} x^{m}$, para todo $x \in L$ e todos $m, n \in \mathbb{Z}$.

4. L associa potências, isto é, $\left(x^{m} x^{n}\right) x^{p}=x^{m}\left(x^{n} x^{p}\right)$, para todo $x \in L$ e todos $m, n, p \in \mathbb{Z}$.

\section{Demonstração.}

1. Um elemento $c \in L$ pertence a $C$ se e somente se $c x=x c$ para todo $x \in L$. Em outras palavras $c \in C$ se e somente se $c=L_{x}^{-1}\left(R_{x}(c)\right)=T_{x}(c)$ para todo $x \in L$. Como L é um A-loop, do lema anterior segue que $C$ é um subloop de $L$. 
2. Temos que $a \in L$ está em $A$ se e somente se $(a x) y=a(x y),(x a) y=x(a y)$ e $x(y a)=(y x)$ a para quaisquer $x, y \in L$. Ou seja $a \in A$ se e somente se $R_{(x, y)}(a)=a, R_{y}^{-1} L_{x}^{-1} R_{y} L_{x}(a)=a$ e $L_{(x, y)}(a)=$ a para todos $x, y \in L$. Notemos que $R_{y}^{-1} L_{x}^{-1} R_{y} L_{x}=T_{y}^{-1} L_{(y, x)}^{-1} T_{x y} R_{(x, y)} T_{x}^{-1} \in \mathcal{I}(L)$. Assim, pelo lema anterior temos que A é um subloop de $L$.

3. Seja $x \in G$, Como $\{x\}$ é um conjunto comutativo de L, existe $C$ subloop comutativo maximal de $L$ que contém $\{x\}$, donde segue que $x^{m} x^{n}=x^{n} x^{m}$ para todos $m, n \in \mathbb{Z}$.

4. Para $x \in L$ temos, do item anterior, que $\{x\}$ está contido num subloop comutativo maximal de $L$, e então $(x x) x=x(x x)$. Ou seja $\{x\}$ é um conjunto associativo de L, logo está contido num subloop associoativo maximal de $L$. Portanto $\left(x^{m} x^{n}\right) x^{p}=x^{m}\left(x^{n} x^{p}\right)$ para todos $m, n, p \in \mathbb{Z}$.

Lema 2.1.6. Sejam $L$ um A-loop e $f, g \in \mathcal{M}(L)$. Suponha $f(1)=g(1)$ e $f(a)=g(a)$ para $a \in G$. Então $f\left(a^{n}\right)=g\left(a^{n}\right)$ para todo $n \in \mathbb{Z}$.

Demonstração. Para $f, g \in \mathcal{M}(L)$, tal que $f(1)=g(1)$, temos $g^{-1}(f(1))=1$, isto é $g^{-1} f \in \mathcal{I}(L)$. Como $g^{-1}(f(a))=$ a, pelo lema 2.1.4, a pertence ao subloop de $L$ que consiste nos elementos que são fixos pelo automorfismo $g^{-1} f$. $\operatorname{Logo~}^{-1}\left(f\left(a^{n}\right)\right)=a^{n}$, para todo $n \in \mathbb{Z}$.

Teorema 2.1.7. Seja L um A-loop. Então:

1. $x^{m}\left(x^{n} y\right)=x^{n}\left(x^{m} y\right)$ para todos $x, y \in L$ e $m, n \in \mathbb{Z}$.

2. $\left(y x^{m}\right) x^{n}=\left(y x^{n}\right) x^{m}$ para todos $x, y \in L$ e $m, n \in \mathbb{Z}$.

3. $\left(x^{m} y\right) x^{n}=x^{m}\left(y x^{n}\right)$ para todos $x, y \in L$ e $m, n \in \mathbb{Z}$.

4. Se $(x y) z=x(y z)$, então $\left(x^{m} y^{n}\right) z^{p}=x^{m}\left(y^{n} z^{p}\right)$ para todos $m, n, p \in \mathbb{Z}$.

5. Se $x y=y x$, então $x^{m} y^{n}=y^{n} x^{m}$ para todos $m, n \in \mathbb{Z}$. 
6. $x[(y x)(z x)]=[(x y)(x z)] x$ para todos $x, y, z \in L$.

Demonstração. Sejam $x, y \in L$

1. A equação $R_{x y}(z)=L_{x} R_{y}(z)$ é satisfeita por $z=1$ e por $z=x$. Então pelo lema 2.1.6 temos que $R_{x y}\left(x^{n}\right)=L_{x} R_{y}\left(x^{n}\right)$, ou seja $x^{n}(x y)=x\left(x^{n} y\right)$, para todo $n \in \mathbb{Z}$. Fixe $n \in \mathbb{Z}$ arbitrariamente e considere a equação $R_{x^{n} y}(z)=L_{x^{n}} R_{y}(z)$. Esta última equação é satisfeita por $z=1$ e por $z=x$, portanto $R_{x^{n} y}\left(x^{m}\right)=$ $L_{x^{n}} R_{y}\left(x^{m}\right)$, para todo $m \in \mathbb{Z}$. Ou seja $x^{m}\left(x^{n} y\right)=x^{n}\left(x^{m} y\right)$ para todos $m, n \in \mathbb{Z}$

2. Temos que $z=1$ e $z=x$ são soluções da equação $L_{y x}(z)=R_{x} L_{y}(z)$, logo, pelo lema 2.1.6 temos que $(y x) x^{n}=\left(y x^{n}\right) x$ para todo $n \in \mathbb{Z}$. Fixado $n \in \mathbb{Z}$ arbitrário, temos que $z=1$ e $z=x$ são soluções de $L_{y x^{n}}(z)=R_{x^{n}} L_{y}(z)$, e novamente pelo lema 2.1.6 temos $\left(y x^{n}\right) x^{m}=\left(y x^{m}\right) x^{n}$ para todos $n, m \in \mathbb{Z}$.

3. Para provar esse ítem, basta seguir os mesmos passos da demonstração dos ítens 1 e 2, mas considerando a equação $L_{x y}(z)=R_{x} R_{y}(z)$.

4. Se $(x y) z=x(y z)$ temos que $L_{x y}(z)=L_{x} L_{y}(z)$. Como $L_{x y}(1)=L_{x} L_{y}(1)$ temos que $L_{x y}\left(z^{p}\right)=L_{x} L_{y}\left(z^{p}\right)$, para todo $p \in \mathbb{Z}$, ou seja $(x y) z^{p}=x\left(y z^{p}\right)$. Assim, temos que $R_{z^{p}} L_{x}(y)=L_{x} R_{z^{p}}(y)$ e então, devido a $R_{z^{p}} L_{x}(1)=L_{x} R_{z^{p}}(1)$, temos $\left(x y^{m}\right) z^{p}=x\left(y^{m} z^{p}\right)$, para quaisquer $m, p \in \mathbb{Z}$. Agora, desde que $R_{z^{p}} R_{y^{m}}(x)=$ $R_{y^{m} z^{p}}(x)$ e $R_{z^{p}} R_{y^{m}}(1)=R_{y^{m} z^{p}}(1)$ temos $\left(x^{n} y^{m}\right) z^{p}=x^{n}\left(y^{m} z^{p}\right)$, para todos $n, m, p \in \mathbb{Z}$.

5. Do mesmo modo que o item anterior, se $x y=y x$, temos a equação $R_{y}(z)=$ $L_{y}(z)$ satisfeita por $z=1$ e $z=x$, portanto $y x^{n}=x^{n} y$ para todo $n \in \mathbb{Z}$. Agora temos $R_{x^{n}}(y)=L_{x^{n}}(y)$ e assim, como $R_{x^{n}}(1)=L_{x^{n}}(1)$, temos $y^{m} x^{n}=x^{n} y^{m}$ para quaisquer $m, n \in \mathbb{Z}$

6. Temos que $T_{x}=L_{x}^{-1} R_{x}$ é um automorfismo de $L$ temos que, para todo $y \in L$,

$$
T_{x} L_{x}(y)=T_{x}(y x)=T_{x}(x) T_{x}(y)=x T_{x}(y)=L_{x} T_{x}(y)
$$


Assim $L_{x} L_{x}^{-1} R_{x}=L_{x}^{-1} R_{x} L_{x}$, isto é, $R_{x} L_{x}=L_{x} R_{x}$ para todo $x \in L$. Desta última igualdade, temos que $T_{x}=L_{x}^{-1} R_{x}=R_{x} L_{x}^{-1}$ e, portanto, para quaisquer $y, z \in L$,

$$
\left(R_{x} L_{x}^{-1}(y)\right)\left(R_{x} L_{x}^{-1}(z)\right)=T_{x}(y) T_{x}(z)=T_{x}(y z)=R_{x} L_{x}^{-1}(y z)
$$

Na equação 2.1, substitua y por xy e z por $x z$, e então termos

$$
\begin{gathered}
\left(R_{x} L_{x}^{-1}(x y)\right)\left(R_{x} L_{x}^{-1}(x z)\right)=R_{x} L_{x}^{-1}((x y)(x z)) \\
R_{x}(y) R_{x}(z)=R_{x} L_{x}^{-1}((x y)(x z)) \\
(y x)(z x)=L_{x}^{-1} R_{x}((x y)(x z)) \\
(y x)(z x)=L_{x}^{-1}([(x y)(x z)] x)
\end{gathered}
$$

e, finalmente,

$$
x[(y x)(z x)]=[(x y)(x z)] x
$$

para todos $x, y, z \in L$.

Consideremos $\mathcal{M}_{\rho}(L)=\left\langle R_{x}: x \in L\right\rangle$ e $\mathcal{M}_{\lambda}(L)=\left\langle L_{x}: x \in L\right\rangle$ subgrupos de $\mathcal{M}(L)$.

Teorema 2.1.8. Seja L um A-loop. Então.

1. Para cada $x \in L$, o conjunto $\left\{R_{x^{m}}, L_{x^{n}}: n, m \in \mathbb{Z}\right\}$ gera um grupo abeliano.

2. $\mathcal{M}_{\rho}(L)$ e $\mathcal{M}_{\lambda}(L)$ são subgrupos normais de $\mathcal{M}(L)$.

\section{Demonstração.}

1. Seja $x \in L$. Do teorema 2.1.7 temos que, para todo $m, n \in \mathbb{Z}, L_{x^{n}} R_{x^{m}}=$ $R_{x^{m}} L_{x^{n}}, L_{x^{n}} L_{x^{m}}=L_{x^{m}} L_{x^{n}}$ e $R_{x^{n}} R_{x^{m}}=R_{x^{m}} R_{x^{n}}$. Portanto $\left\langle R_{x^{m}}, L_{x^{n}}: n, m \in\right.$ $\mathbb{Z}\rangle$ é um grupo abeliano. 
2. Sejam $x, y \in L$. Segue do item 6 do teorema 2.1.7 que $x[(y x)(z x)]=[(x y)(x z)] x$ para todo $z \in L$. Então $L_{x} L_{y x} R_{x}=R_{x} L_{x y} L_{x}$, ou seja $L_{x} L_{y x} L_{x}^{-1}=R_{x} L_{x y} R_{x}^{-1}$. Fixando $x$ e fazendo y variar em $L$, temos que $R_{x} \mathcal{M}_{\lambda}(L) R_{x}^{-1} \subset \mathcal{M}_{\lambda}$ para todo $x \in L$. Como $\mathcal{M}(L)=\left\langle\mathcal{M}_{\lambda}(L), \mathcal{M}_{\rho}(L)\right\rangle$, temos que $\mathcal{M}_{\lambda}(L)$ é normal em $\mathcal{M}(L)$.

Novamente de $x[(y x)(z x)]=[(x y)(x z)] x$ temos que $L_{x} R_{z x} R_{x}=R_{x} R_{x z} L_{x}$, que é o mesmo que $R_{x}^{-1} R_{z x} R_{x}=L_{x}^{-1} R_{x z} L_{x}$ para todo $x, z \in L$. De modo análogo ao acima temos que $\mathcal{M}_{\rho}(L)$ é um subgrupo normal de $\mathcal{M}(L)$.

Para $x, y \in L$, definimos $C(x, y)=L_{y}^{-1} R_{x}^{-1} L_{y} R_{x}$. Como

$$
C(x, y)=\left(L_{y}^{-1} R_{y}\right)\left(R_{y}^{-1} R_{x}^{-1} R_{y x}\right)\left(R_{y x}^{-1} L_{y x}\right)\left(L_{y x}^{-1} L_{y} L_{x}\right)\left(L_{x}^{-1} R_{x}\right)=T_{y} R_{(y, x)}^{-1} T_{y x}^{-1} L_{(x, y)} T_{x},
$$

temos que $C(x, y) \in \mathcal{I}(L)$ para todos $x, y \in L$.

Considere $\mathcal{I}_{\rho}(L)=\left\langle R_{(x, y)}: x, y \in L\right\rangle, \mathcal{I}_{\lambda}(L)=\left\langle L_{(x, y)}: x, y \in L\right\rangle$ e $\mathcal{I}_{\mu}(L)=$ $\langle C(x, y): x, y \in L\rangle$ subgrupos de $\mathcal{I}(L)$. Para cada $a \in L$, temos que $a \in \mathcal{N}_{\rho}(L)$ se e somente se $(x y) a=x(y a)$, para todos $x, y \in L$ que é equivalente a $L_{x y}^{-1} L_{x} L_{y}(a)=a$ para todos $x, y \in L$. Em outras palavras $a \in \mathcal{N}_{\rho}(L)$ se e somente se $f(a)=a$ para toda $f \in \mathcal{I}_{\lambda}(L)$. Do mesmo modo, temos $a \in \mathcal{N}_{\lambda}(L)$ se e somente se $f(a)=a$ para toda $f \in \mathcal{I}_{\rho}(L)$ e $a \in \mathcal{N}_{\mu}(L)$ se e somente se $f(a)=a$ para toda $f \in \mathcal{I}_{\mu}(L)$.

Lema 2.1.9. Seja L um A-loop. Então

1. $C(x, y)=T_{x}^{-1} L_{(y, x)}^{-1} L_{(x, y)} T_{x}$ para quaisquer $x, y \in L$.

2. $C(x, y)=T_{y} R_{(y, x)}^{-1} R_{(x, y)} T_{y}^{-1}$ para quaisquer $x, y \in L$.

3. $C(x, y)=T_{y} R_{(y, x)}^{-1} T_{y x}^{-1} L_{(x, y)} T_{x}$ para quaisquer $x, y \in L$.

Demonstração. Temos que o ítem 3 foi demonstrado acima. Vamos agora mostrar os itens 1 e 2.

1. Temos que $C(x, y)=L_{y}^{-1} T_{x}^{-1} L_{x}^{-1} L_{y x} L_{(x, y)} T_{x}$ e, como $T_{x}$ é um automorfismo de $L$ pela equação 1.2 temos $C(x, y)=L_{y}^{-1} L_{T_{x}^{-1}(x)}^{-1} L_{T_{x}^{-1}(y x)} T_{x}^{-1} L_{(x, y)} T_{x}$, ou seja

$$
C(x, y)=L_{(y, x)}^{-1} T_{x}^{-1} L_{(x, y)} T_{x}
$$


pois $T_{x}^{-1}(x)=x$ e $T_{x}^{-1}(y x)=x y$. Por outro lado, temos $L_{(y, x)}(x)=L_{x y}^{-1}(x(y x))=$ $L_{x y}^{-1}((x y) x)=x$, desde que, pelo ítem 3 do teorema 2.1.7 temos $x(y x)=$ $(x y) x$. Como $L_{(y, x)}$ é um automorfismo de $L$ temos $L_{(y, x)}(z x)=L_{(y, x)}(z) \cdot x$, para todo $z \in L$, isto é, $L_{(y, x)} R_{x}=R_{x} L_{(y, x)}$ para todo $x, y \in L$. Agora, fazendo $L_{(y, x)}(x z)=x . L_{(y, x)}(z)$, obtemos $L_{(y, x)} L_{x}=L_{x} L_{(y, x)}$ para todo $x, y \in L$. Daí segue que $L_{(y, x)} T_{x}=T_{x} L_{(y, x)}$, e então, na equação 2.2 temos $C(x, y)=$ $T_{x}^{-1} L_{(y, x)}^{-1} L_{(x, y)} T_{x}$ para quaisquer $x, y \in L$.

2. Podemos escrever $C(x, y)=T_{y} R_{y}^{-1} R_{x}^{-1} T_{y}^{-1} R_{y} R_{x}=T_{y} R_{y}^{-1} R_{x}^{-1} T_{y}^{-1} R_{x y} T_{y} T_{y}^{-1} R_{x y}^{-1} R_{y} R_{x}$.

Ou seja, $C(x, y)=T_{y} R_{(y, x)}^{-1} T_{y}^{-1} R_{(x, y)}$, pois $T_{y}^{-1}(x y)=y x$. Como $R_{(x, y)} T_{y}=$ $T_{y} R_{(x, y)}$, para todo $x, y \in L$, obtemos $C(x, y)=T_{y} R_{(y, x)}^{-1} R_{(x, y)} T_{y}^{-1}$ para quaisquer $x, y \in L$.

Corolário 2.1.10. Se $L$ for um A-loop então $\mathcal{N}_{\rho}(L) \subset \mathcal{N}_{\mu}(L)$ e $\mathcal{N}_{\lambda}(L) \subset \mathcal{N}_{\mu}(L)$. Em particular, $\mathcal{N}(L)=\mathcal{N}_{\rho}(L) \cap \mathcal{N}_{\lambda}(L)$

Demonstração. Basta observar que, pelos ítens 1 e 2 do lema 2.1.9 e pelas equações 1.2 e 1.3 temos

$$
C(x, y)=L_{\left(T_{x}(y)^{-1}, T_{x}(x)^{-1}\right)}^{-1} L_{\left(T_{x}(x)^{-1}, T_{x}(y)^{-1}\right)}=R_{\left(T_{y}(y), T_{y}(x)\right)}^{-1} R_{\left(T_{y}(x), T_{y}(y)\right)},
$$

para quaisquer $x, y \in L$.

\section{$2.2 \quad A$-loops Diassociativos}

Definição 2.2.1. Um loop $L$ é chamado diassociativo se, para quaisquer $x, y \in L$ o subloop gerado por $x, y,\langle x, y\rangle$, for associativo (e, portanto, um grupo).

Teorema 2.2.2. Seja L um A-loop. As seguintes propriedades são equivalentes.

1. L é diassociativo.

2. $(y x) x=y x^{2}$ e $x(x y)=x^{2} y$, para quaisquer $x, y \in L$. 
3. L é um PI-loop.

As identidades do ítem 2 do teorema 2.2.2 são chamadas de leis alternativas.

Demonstração. Supondo o item 2 verdadairo, vamos provar o ítem 1. Como, pelo teorema 2.1.7, $(x y) x=x(y x)$ para quaisquer $x, y$ em qualquer A-loop L, temos que $\{x, y\}$ é um subconjunto associativo de L. Logo está contido num subconjunto associativo maximal de L, que é um grupo. Assim, $\langle x, y\rangle$ é um grupo.

Agora vamos ter por hipótese o ítem 3. Temos que cada elemento $x \in L$ possui um inverso bilateral $x^{-1}$ e que $x^{-1}(x y)=y=(y x) x^{-1}$ para todo $y \in L$. Do ítem 4 do teorema 2.1.7, temos que $x^{-1}(x y)=y=\left(x^{-1} x\right) y$ e $(y x) x^{-1}=y=y\left(x x^{-1}\right)$ implicam que $x(x y)=x^{2} y$ e que $(y x) x=y x^{2}$, o que prova o item 2.

Assumindo o item 1, vale que $\langle x, y\rangle$ é um grupo, para quaisquer $x, y \in L$. Assim $x^{\lambda}=x^{\rho}=x^{-1} \in\langle x, y\rangle$ e $x^{-1}(x y)=y=(y x) x^{-1}$. Com isso, provamos o item 3, o que completa a demonstração.

Teorema 2.2.3. Seja $L$ um A-loop diassociativo e sejam $a, b, c \in L$ tais que $(a b) c=$ $a(b c)$. Então o subloop $H=\langle a, b, c\rangle$ é associativo.

Demonstração. Nosso objetivo é mostrar que os elementos a, b, c associam-se em qualquer ordem. Com isso $\{a, b, c\}$ será um subconjunto associativo de $L$ e estará contido num subgrupo de L.

Considere o conjunto $S=\{s \in H:(a b) s=a(b s)\}$. Como L é um A-loop, $H$ também o é, e assim $S$ é um subloop de $H$, pois $s \in S$ se e somente se $L_{(b, a)}(s)=s$. Mas, note que $c \in S$, por hipótese e $a, b \in S$ pela diassociatividade. Logo $S=H$ e

$$
(a b) h=a(b h) \quad \text { para todo } \quad h \in H .
$$

Em 2.3, faça $h=b^{-1} c$, então $(a b)\left(b^{-1} c\right)=a\left(b\left(b^{-1} c\right)\right)=a c$ por diassociatividade. Consequentemente, $a b=(a c)\left(b^{-1} c\right)^{-1}=(a c)\left(c^{-1} b\right)$, e assim

$$
a b=a\left(c\left(c^{-1} b\right)\right)=(a c)\left(c^{-1} b\right) .
$$


É fato que $H=\left\langle a, c, c^{-1} b\right\rangle$ e por 2.4 temos que

$$
(a c) h=a(\text { ch }) \quad \text { para todo } \quad h \in H .
$$

As outras formas de associatividade seguem usando o mesmo raciocínio. Sendo $\{a, b, c\}$ um subconjunto associativo de $L$, a demonstração está completa.

Seja $L$ um $A$-loop diassociativo. Vimos que, $L$ é um $P I$-loop e então consideremos $J: L \longrightarrow L$ a aplicação definida por $J(x)=x^{-1}$. Vimos na proposição 1.2.2 que vale

$$
\begin{gathered}
L_{x}^{-1}=L_{x^{-1}}, \quad R_{x}^{-1}=R_{x^{-1}}, \\
J L_{x} J=R_{x^{-1}} \quad \text { e } \quad J R_{x} J=L_{x^{-1}}
\end{gathered}
$$

para todo $x \in L$. Se $\theta$ for um automorfismo de $L$, temos que

$$
J \theta(x)=(\theta(x))^{-1}=\theta\left(x^{-1}\right)=\theta J(x),
$$

para todo $x \in L$, ou seja $J \theta=\theta J$. Agora, em relação aos geradores de $\mathcal{I}(L)$, temos

$$
\begin{gathered}
L_{(x, y)}=L_{(x, y)} J^{2}=J L_{(x, y)} J=J L_{y x}^{-1} L_{y} L_{x} J=R_{(y x)^{-1}}^{-1} R_{y^{-1}} R_{x^{-1}}=R_{\left(x^{-1}, y^{-1}\right)}, \\
R_{(x, y)}=R_{(x, y)} J^{2}=J R_{(x, y)} J=J R_{x y}^{-1} R_{y} R_{x} J=L_{(x y)^{-1}}^{-1} L_{y^{-1}} L_{x^{-1}}=L_{\left(x^{-1}, y^{-1}\right)}, \\
T_{x}=J T_{x} J=J L_{x}^{-1} J J R_{x} J=R_{x} L_{x}^{-1}=R_{x^{-1}}^{-1} L_{x}^{-1}=\left(L_{x} R_{x^{-1}}\right)^{-1}=T_{x^{-1}}^{-1}, \\
C(x, y)=J C(x, y) J=J R_{x} L_{y} R_{x}^{-1} L_{y}^{-1} J=C\left(y^{-1}, x^{-1}\right)^{-1} .
\end{gathered}
$$

Observação 2.2.4. Para $p, q, r$, no subloop gerado por $\{x, y\}$, temos que $R_{(q, r)}(p)=$ $p$, pois $L$ é um loop diassociativo. Assim $R_{(q, r)}(z p)=R_{(q, r)}(z) R_{(q, r)}(p)=R_{(q, r)}(z) p$ para qualquer $z \in L$, logo

$$
R_{p} R_{(q, r)}=R_{(q, r)} R_{p} .
$$

Lema 2.2.5. Sejam L um A-loop diassociativo e $p, q, r$ elementos do subloop $\langle x, y\rangle$. Então:

1. $R_{p} R_{(q, r)}=R_{(q, r)} R_{p}$ e $L_{p} R_{(q, r)}=R_{(q, r)} L_{p}$; 
2. $R_{p} L_{(q, r)}=L_{(q, r)} R_{p}$ e $L_{p} L_{(q, r)}=L_{(q, r)} L_{p}$

3. $R_{p} C(q, r)=C(q, r) R_{p}$ e $L_{p} C(q, r)=C(q, r) L_{p}$;

4. $R_{(q, r)}=R_{\left(p q r,(q r)^{-1)}\right.} R_{(p q, r)} R_{(p, q)}$;

5. $L_{(q, r)}=L_{\left(r q p,(r q)^{-1}\right) L_{(q p, r)}} L_{(p, q)}$.

\section{Demonstração.}

1. Vimos que $R_{p} R_{(q, r)}=R_{(q, r)} R_{p}$ (equação 2.10). Para $z \in L$ temos, pela observação 2.2.4,

$$
R_{(q, r)} L_{p}(z)=R_{(q, r)}(p z)=R_{(q, r)}(p) R_{(q, r)}(z)=p R_{(q, r)}(z)=L_{p} R_{(q, r)}(z),
$$

donde segue $R_{(q, r)} L_{p}=L_{p} R_{(q, r)}$.

2. Como $L_{(q, r)}(p)=L_{(r q)^{-1}} L_{r} L_{q}(p)=p$, segue que, do mesmo modo feito no item 1, $L_{(q, r)} R_{p}=R_{p} L_{(q, r)}$ e $L_{(q, r)} L_{p}=L_{p} L_{(q, r)}$.

3. Vamos escrever $T_{q}(p)=L_{q^{-1}} R_{q}(p)=q^{-1} p q$ e $T_{q}^{-1}=R_{q^{-1}} L_{q}(p)=q p q^{-1}$. Do fato que $\left.C(q, r)=T_{r} R_{(r, q)}^{-1} T_{(} r q\right)^{-1} L_{(q, r)} T_{q}$ temos que

$$
\begin{aligned}
C(q, r)(p) & =T_{r} R_{(r, q)}^{-1} T(r q)^{-1} L_{(q, r)}\left(q^{-1} p q\right) \\
& =T_{r} R_{(r, q)}^{-1} T_{(r q)^{-1}\left(q^{-1} q\right)} \\
& =T_{r} R_{(r, q)}^{-1}\left(r p r^{-1}\right) \\
& =T_{r}\left(r p r^{-1}\right)=p .
\end{aligned}
$$

Assim

$C(q, r) R_{p}(z)=C(q, r)(z p)=C(q, r)(z) C(q, r)(p)=C(q, r)(z) p=R_{p} C(q, r)(z)$

$e$

$C(q, r) L_{p}(z)=C(q, r)(p z)=C(q, r)(p) C(q, r)(z)=p C(q, r)(z)=L_{p} C(q, r)(z)$,

para todo $z \in L$. Logo $R_{p} C(q, r)=C(q, r) R_{p}$ e $L_{p} C(q, r)=C(q, r) L_{p}$. 
4. Do item 1, obtemos

$$
\begin{aligned}
R_{(q, r)} & =R_{p^{-1}} R_{(q, r)} R_{p} \\
& =R_{p^{-1}} R_{q r}^{-1} R_{r} R_{q} R_{p} \\
& =R_{p^{-1}} R_{q r}^{-1} R_{r} R_{p q} R_{p q}^{-1} R_{q} R_{p} \\
& =R_{p^{-1}} R_{q r}^{-1} R_{r} R_{p q} R_{(p, q)} \\
& =R_{p^{-1}} R_{q r}^{-1} R_{p q r} R_{p q r}^{-1} R_{r} R_{p q} R_{(p, q)} \\
& =R_{p^{-1} q r\left(q r^{-1}\right)} R_{q r}^{-1} R_{p q r} R_{(p q, r)} R_{(p, q)} \\
& =R_{\left(p q r,\left(q r^{-1}\right)\right)} R_{(p q, r)} R_{(p, q)} .
\end{aligned}
$$

5. A partir do item 2, obtems 5 do mesmo modo que obtemos o item 4.

Do ítem 1 do lema 2.2.5 temos $R_{(q, r)}^{-1}=R_{p}^{-1} R_{(q, r)}^{-1} R_{p}$, para quaisquer $p, q, r$ no subloop $\langle x, y\rangle$. Fazendo $p=x y, q=x$ e $r=y$, obtemos $R_{(x, y)}^{-1}=R_{\left(y^{-1}, x^{-1}\right)}$. Da mesma forma $L_{(x, y)}^{-1}=L_{\left(y^{-1}, x^{-1}\right)}$.

\subsection{Isotopias de $A$-Loops}

Nessa e na próxima seção, trataremos de como obter "novos" $A$-loops a partir de um $A$-loop dado. Durante tais seções, $L$, denotará o loop $(L,$.$) . Aqui, nosso objetivo$ é determinar condições para que um isótopo de $L$ também seja um $A$-loop.

Definição 2.3.1. Um loop $(H, *)$ é dito isótopo do loop L se existirem bijeções $U, V . W: H \longrightarrow L$, tais que

$$
W(u * v)=U(u) V(v) \quad \text { para quaisquer } \quad u, v \in H .
$$

Um loop $(L, \circ)$ é dito um isótopo principal do loop L, se existirem $P, Q$, permutações do conjunto L, tais que

$$
x \circ y=P(x) Q(y) \quad \text { para quaisquer } \quad x, y \in L .
$$


Em $[\mathrm{Br}]$ temos que todo loop isótopo de $L$ é isomorfo a um isótopo principal $(L, \circ)$, cuja mltiplicação é dada por

$$
x \circ y=R_{f}^{-1}(x) L_{g}^{-1}(y)
$$

onde $f, g$ são elemntos fixados de $L$ e, além disso, $g f$ é a identidade de $(L, \circ)$.

Agora vamos considerar $(L, *)$ o isótopo principal de $L$ definido por

$$
x * y=R_{f}^{-1}(x) y \quad \text { para todos } \quad x, y \in L .
$$

Se definirmos o loop $(L, \circ)$ por

$$
R_{f}(x \circ y)=R_{f}(x) * R_{f}(y)
$$

então $(L, \circ)$ e $(L, *)$ serão isomorfos e, além disso,

$$
x \circ y=R_{f}^{-1}\left(x R_{f}(y)\right) \quad \text { para quaisquer } \quad x, y \in L .
$$

Afirmamos que $L$ e $(L, \circ)$ têm a mesma identidade e $\mathcal{M}(L)=\mathcal{M}(L, \circ)$. De fato, para mostrar que 1 é a identidade de $(L, \circ)$, basta observar que

$$
x \circ 1=R_{f}^{-1}\left(x R_{f}(1)\right)=R_{f}^{-1}\left(R_{f}(x)\right)=x
$$

e

$$
1 \circ x=R_{f}^{-1}\left(1 R_{f}(x)\right)=x
$$

- Para $x \in L$, denotaremos por $L_{x}^{\circ}$ e $R_{x}^{\circ}$ as translações à esquerda e à direita, respectivamente, em relação a $(L, \circ)$. Para qualquer que seja $y \in L$, temos

$$
L_{x}^{\circ}(y)=x \circ y=R_{f}^{-1}\left(x R_{f}(y)\right)=R_{f}^{-1} L_{x} R_{f}(y)
$$

$\mathrm{e}$

$$
R_{x}^{\circ}(y)=y \circ x=R_{f}^{-1}\left(y R_{f}(x)\right)=R_{f}^{-1}\left(y L_{x}(f)\right)=R_{f}^{-1} R_{L_{x}(f)}(y)
$$


- Logo $\mathcal{M}(L, o) \subset \mathcal{M}(L)$. Para inclusão contrária, usaremos o fato que existem únicos $u, v \in L$ tais que $x=u f$ e $y=v f$ e, ainda, de $x * y=R_{f}^{-1}(x) y$ e $R_{f}(x \circ y)=$ $R_{f}(x) * R_{f}(y)$ obtemos

$$
x y=R_{f}^{-1} R_{f}(x) y=R_{f}(x) * y=R_{f}\left(x \circ R_{f}^{-1}(y)\right)=R_{f} R_{v}^{\circ}(x),
$$

ou seja, $R_{f}^{-1} R_{y}=R_{v}^{\circ}$. Logo, para cada $y \in L, R_{f}^{-1} R_{y} \in \mathcal{M}(L, \circ)$. Fazendo $y=1$ temos $R_{f}^{-1} \quad$ (e, portanto) $\quad R_{f} \in \mathcal{M}(L, \circ)$, assim $R_{y} \in \mathcal{M}(L, \circ)$ para todo $y \in L$. Da memsa forma, $L_{y} \in \mathcal{M}(L, \circ)$ para todo $y \in L$. Portanto

$$
\mathcal{M}(L)=\mathcal{M}(L, \circ)
$$

Como consequência de 2.17 e do fato que as identidades de $L$ e $(L, \circ)$ coincidem, segue $\mathcal{I}(L)=\mathcal{I}(L, \circ)$.

A partir de agora suponha $L$ um $A$-loop. Se $(L, \circ)$, definido como em 2.16, também for um $A$-loop então para todo $U \in \mathcal{I}(L)$ vale que $U(x \circ y)=U(x) \circ U(y)$, $\mathrm{ou}$

$$
U(x \circ y)=U\left(R_{f}^{-1}\left(x R_{f}(y)\right)\right)=R_{U(f)}^{-1}\left(U(x) R_{f}(y)\right)=R_{U(f)}^{-1}\left(U\left(x R_{U(f)}(U(y))\right)\right),
$$

$\mathrm{Ou}$

$$
R_{f}^{-1}\left(U(x) R_{f}(U(y))\right)=R_{U(f)}^{-1}\left(U(x) R_{U(f)}(U(y))\right) \quad \text { para todos } \quad x, y \in L .
$$

Substituindo, em 2.18, $x$ por $U^{-1}(x), y$ por $U^{-1} R_{f}^{-1}(y)$ e chamando $S=R_{U(f) R_{f}^{-1}}$ obtemos

$$
S(x y)=x S(y) \quad \text { para todos } \quad x, y \in L .
$$

Agora, faça $y=1$, em 2.19, e denote $s=S(1)$. Então $S(x)=x s=R_{s}(x)$, qualquer que seja $x \in L$, isto é $S=R_{s}$. Voltando a 2.19, temos $R_{s}(x y)=x R_{s}(y)$ para todos $x, y \in L$, o que é equivalente a $s \in \mathcal{N}_{\rho}(L)$.

Concluimos que se $(L, \circ)$ for um $A$-loop então para cada $U \in \mathcal{I}(L)$ existirá $s \in \mathcal{N}_{\rho}(L)$ tal que $R_{U(f)}=R_{s} R_{f}$. 
Reciprocamente, suponha que para cada $U \in \mathcal{I}(L)$ exista $s \in \mathcal{N}_{\rho}(L)$ tal que $R_{U(f)}=R_{s} R_{f}$. Como, para quaisquer $x, y \in L$, temos que $R_{s}(x y)=x R_{s}(y)$, vale que

$$
\begin{aligned}
U(x \circ y) & =U\left(R_{f}^{-1}\left(x R_{f}(y)\right)\right) \\
& =R_{U(f)}^{-1}\left(U(x) R_{U(f)}(U(y))\right) \\
& =R_{U(f)}^{-1}\left(U(x) R_{s} R_{f}(U(y))\right) \\
& =R_{U(f)}^{-1} R_{s}\left(U(x) R_{f}(U(y))\right) \\
& =R_{f}^{-1}\left(U(x) R_{f}(U(y))\right) \\
& =U(x) \circ U(y)
\end{aligned}
$$

donde segue que $U \in \operatorname{Aut}(L, \circ)$. Com isso, temos demosntrado o seguinte lema:

Lema 2.3.2. Seja L um A-loop. Uma codição necessária e suficiente para que o iótopo principal $(L, \circ)$, definido por $x \circ y=R_{f}^{-1}\left(x R_{f}(y)\right)$, para $x, y \in L$, seja um A-loop é que, para cada $U \in \mathcal{I}(L)$, exista $s \in \mathcal{N}_{\rho}(L)$ tal que $R_{U(f)}=R_{s} R_{f}$.

Observação. No lema acima, a condição $R_{U(f)}=R_{s} R_{f}$ é equivaente a $L_{f}^{-1}(U(f)) \in$ $\mathcal{N}_{\rho}(L)$.

O subloop (centralmente) derivado, $L^{\prime}$, é o subloop de $L$, definido pela interseção de todos os subloops $K \triangleleft L$, tais que $L / K$ seja um grupo abeliano. Em [Br], Bruck provou que

$$
L^{\prime}=\left\langle R_{x}^{-1}(U(x)): x \in L, U \in \mathcal{I}(L)\right\rangle=\left\langle L_{x}^{-1}(U(x)): x \in L, U \in \mathcal{I}(L)\right\rangle .
$$

Assim, de 2.20, temos que um isótomo principal de um $A$-loop $L$ do tipo 2.16 será um $A$-loop se e somente se $L^{\prime} \subset \mathcal{N}_{\rho}(L)$. Definindo $(L, \star)$, isótopo principal de um $A$-loop $L$, por

$$
x \star y=L_{g}^{-1}\left(L_{g}(x) y\right) \quad \text { para quaisquer } \quad x, y \in L,
$$

temos que, $(L, \star)$ será um $A$-loop se e somente se $L^{\prime} \subset \mathcal{N}_{\lambda}(L)$.

A seguir, temos o principal teorema dessa seção. 
Teorema 2.3.3. Seja L um A-loop. Então todo loop isótopo de L será um A-loop se e somente se $L^{\prime} \subset \mathcal{N}(L)$.

Demonstração. Se todos os isótopos de L são A-loops, em particular os isótopos dos tipos 2.16 e 2.21, também o são. Então $L^{\prime} \subset \mathcal{N}_{\rho}(L)$ e $L^{\prime} \subset \mathcal{N}_{\lambda}(L)$, donde temos $L^{\prime} \subset \mathcal{N}(L)=\mathcal{N}_{\rho}(L) \cap \mathcal{N}_{\lambda}(L)$.

Reciprocamente suponha $L^{\prime} \subset \mathcal{N}(L)$ e seja $(L, \circ)$ um isótopo de $L$ da forma

$$
x \circ y=R_{f}^{-1}(x) L_{g}^{-1}(y) .
$$

Sabemos que gf é a identidade de $(L, \circ), L_{x}^{\circ}(y)=L_{R_{f}^{-1}(x)} L_{g}(y), R_{y}^{\circ}(x)=R_{L_{g}^{-1}(y)} R_{f}^{-1}(x)$ e $x y=R_{f}^{-1}(x f) L_{g}^{-1}(g y)=R_{f}(x) \circ L_{g}(y)$. Assim $\mathcal{M}(L)=\mathcal{M}(L, \circ)$.

Uma aplicação $T \in \mathcal{M}(L, \circ)$ será uma aplicação interna de $(L, \circ)$ se e somente se $T(g f)=g f$. De $[B r]$ temos que existem $U \in \mathcal{I}(L)$ e $b \in L$ tais que $T=R_{b} U$. Temos que $L_{g f}^{-1}(U(g f))=a \in \mathcal{N}(L)$, então $U(g f)=(g f)$ a. Assim, $T \in \mathcal{I}(L, \circ)$ se e somente se

$$
g f=T(g f)=R_{b} U(g f)=((g f) a) b=(g f)(a b),
$$

ou seja, $b=a^{-1}$. Também temos que $U(f)=f s$ e $U(g)=g t$, onde $s, t \in \mathcal{N}(L)$, assim, $g(f a)=(g f) a=U(g f)=U(g) U(f)=(g t)(f s)=g(t(f s))$, isto é, fa $=(t f) s$. Logo

$$
\begin{aligned}
T\left(T^{-1}(x) \circ T^{-1}(y)\right) & =R_{a^{-1}} U\left(U^{-1} R_{a}(x) \circ U^{-1} R_{a}(y)\right) \\
& =R_{a^{-1}} U\left(\left(R_{f}^{-1} U^{-1} R_{a}(x)\right)\left(L_{g}^{-1} U^{-1} R_{a}(y)\right)\right) \\
& =R_{a^{-1}}\left(U\left(R_{f}^{-1} U^{-1} R_{a}(x)\right) U\left(L_{g}^{-1} U^{-1} R_{a}(y)\right)\right. \\
& =\left(R_{U(f)}^{-1}\left(R_{a}(x)\right) L_{U(g)}^{-1}\left(R_{a}(y)\right)\right) a^{-1} \\
& =\left(R_{f s}^{-1}\left(R_{a}(x)\right) L_{g t}^{-1}\left(R_{a}(y)\right)\right) a^{-1} \\
& =\left(R_{f s}^{-1} R_{a}(x)\right)\left(L_{g t}^{-1} R_{a}(y) \cdot a^{-1}\right) \\
& =\left(R_{f s}^{-1} R_{a}(x)\right)\left(R_{a^{-1}} L_{g t}^{-1} R_{a}(y)\right) \\
& =\left(R_{t^{-1}}^{-1} R_{a}(x)\right) L_{g t}^{-1}(y) \\
& =R_{t} R_{f}^{-1}(x) L_{t}^{-1} L_{g}^{-1}(y) \\
& =R_{f}^{-1}(x) L_{g}^{-1}(y)=x \circ y,
\end{aligned}
$$


onde, em 2.22 usamos o fato de que a $\in \mathcal{N}(L)$. Assim mostramos que $T^{-1}$ é um automorfismo de $(L, \circ)$, consequentemete, $(L, \circ)$ é um A-loop, como queríamos demosntrar.

\subsection{Holomorfos de $A$-loops}

Agora discutiremos condições para que um tipo especial de holomorfo do $A$-loop $L$ também seja um $A$-loop.

Definição 2.4.1. Seja $K^{*}$ um grupo de automorfismos de L. O $K^{*}$-holomorfo de $L$, denotado por $\left[K^{*}, L\right]$, é o loop formado por elementos $[S, x]$, onde $S \in K^{*}$ e $x \in L$, com multiplicação dada por

$$
[S, x][T, y]=[T S, T(x) y]
$$

O conjunto $\left[K^{*}, L\right]$ é, de fato, um loop pois, para quaisquer $[A, a],[B, b] \in$ $\left[K^{*}, L\right]$, temos que $\left[A^{-1} B, A^{-1}\left(R_{a}^{-1}(b)\right)\right]$ e $\left[B A^{-1}, L_{B A^{-1}(a)}(b)\right]$ são, respectivamente, as únicas soluções de $[X, x][A, a]=[B, b]$ e de $[A, a][X, x]=[B, b]$. Além disso, está claro que $\left[I d_{L}, 1\right]$ é o elememto neutro de $\left[K^{*}, L\right]$.

Definição 2.4.2. Um automorfismo de centro de um loop L é um automorfismo $S$ de $L$, tal que $S(x)=x . c(x)$ onde $c(x)$ é um elemento de $\mathcal{Z}(L)$.

Temos que se $S$ for um automorfismo de centro de $L, S^{-1}$ também será. De fato, se $S(x)=x \cdot c(x)$, com $c(x) \in \mathcal{Z}(L)$, então $S^{-1}(x)=S^{-1}\left(S(x) \cdot c(x)^{-1}\right)=$ $x . S^{-1}\left(c(x)^{-1}\right)$. Como $S^{-1}\left(c(x)^{-1}\right) \in \mathcal{Z}(L)$, concluimos que $S^{-1}$ é um automorfismo de centro de $L$.

Lema 2.4.3. Se $S$ for um automorfismo de centro de $L$, então $S U=U S$ para toda aplicação interna $U$.

Demonstração. Notemos que, para quaisquer $x, y \in L$,

$$
(x y) . c(x y)=S(x y)=S(x) S(y)=(x \cdot c(x))(y \cdot c(y))=(x y) \cdot c(x) c(y),
$$


ou seja, $S$ induz um homomorfismo de $L$ no grupo abeliano $\mathcal{Z}(L)$. Pelo Teorema do Homomorfismo, temos que $L / \operatorname{ker}(c) \cong H \leq \mathcal{Z}(L)$, assim, $L^{\prime} \subset \operatorname{ker}(c)$. Como $L^{\prime}=\left\langle L_{x}^{-1}(U(x)): x \in L, U \in \mathcal{I}(L)\right\rangle$, temos que $c\left(L_{x}^{-1} U(x)\right)=1$, quaisquer que sejam $x \in L$ e $U \in \mathcal{I}(L)$. Desde que, $U(x)=L_{x} L_{x}^{-1} U(x)=x . L_{x}^{-1} U(x)$ temos que $c(U(x))=c\left(x \cdot L_{x}^{-1} U(x)\right)=c(x)$.

Usando que $U(x . c(x))=U(x) . c(x)$, fato demonstrado por Bruck em [Br], temos que

$$
U(S(x))=U(x \cdot c(x))=U(x) \cdot c(x)=U(x) \cdot c(U(x))=S(U(x)),
$$

e, portanto, $U S=S U$.

O próximo teorema, o resultado mais importante dessa seção, nos dá condições de caracterizar quando que um $K^{*}$-holomorfo de um loop é um $A$-loop, para um grupo particular de automorfismos, é um $A$-loop.

Teorema 2.4.4. Seja $K^{*}$ um grupo de automorfismos de centro de um loop L. Uma condição necessária e suficiente para que o $K^{*}$-holomorfo $L$ seja um A-loop é que $L$ seja um A-loop.

Demonstração. Suponha $\left[K^{*}, L\right]$ um A-loop.

Desde que o conjunto $\left\{\left[I d_{L}, x\right] \mid x \in L\right\}$ é um subloop de $\left[K^{*}, L\right]$ isomorfo a $L$, temos que L é um A-loop.

Reciprocamente, suponha $L$ um A-loop.

Sejam $[A, a],[B, b] \in\left[K^{*}, L\right]$. Queremos mostrar que $R_{([A, a],[B, b])}, L_{([A, a],[B, b])} e$ $T_{[A, a]}$ são automorfismos de $\left[K^{*}, L\right]$. Para qualquer $[S, x] \in\left[K^{*}, L\right]$ temos que

$$
R_{([A, a],[B, b])}([S, x])=\left[S, R_{(B(a), b)}(x)\right]
$$

$e$

$$
L_{([A, a],[B, b])}([S, x])=\left[S, L_{(a, A(b))}(x)\right] .
$$

Sejam $[S, x],[V, y] \in\left[K^{*}, L\right]$. De 2.24, temos que

$$
R_{([A, a],[B, b])}([S, x][V, y])=\left[V S, V\left(R_{(B(a), b)}(x)\right) R_{(B(a), b)}(y)\right]
$$


e, por outro lado,

$$
R_{([A, a],[B, b])}([S, x])=\left[S, R_{(B(a), b)}(x)\right] \quad e \quad R_{([A, a],[B, b])}([V, y])=\left[V, R_{(B(a), b)}(y)\right]
$$

Assim, concluimos que

$$
R_{([A, a],[B, b])}([S, x][V, y])=R_{([A, a],[B, b])}([S, x]) R_{([A, a],[B, b])}([V, y])
$$

donde temos que $R_{([A, a],[B, b])}$ é um automorfismo de $\left[K^{*}, L\right]$. De maneira análoga temos que $L_{([A, a],[B, b])}$ também é um automorfismo de $\left[K^{*}, L\right]$.

Finalmente vamos analisar o gerador $T_{[A, a]}$. Fazendo

$$
T_{[A, a]}([S, x])=L_{[A, a]}^{-1} R_{[A, a]}([S, x])=[Z, z]
$$

teremos $Z=A S A^{-1}$ e $A(x) a=A S A^{-1}(a) z$. Como $A S A^{-1}$ é um automorfismo de centro de L, temos que $A S A^{-1}(a)=a \cdot c_{A S A^{-1}}(a)$, onde $c_{A S A^{-1}}$ é o homomorfismo de $L$ em $\mathcal{Z}(L)$ induzido por $A S A^{-1}$. Assim

$$
A(x) a=\left(a . c_{A S A^{-1}}(a)\right) z=a\left(c_{A S A^{-1}}(a) z\right)
$$

ou seja, $z=T_{a}(A(x)) \cdot c_{A S A^{-1}}(a)^{-1}$, portanto

$$
T_{[A, a]}([S, x])=\left[A S A^{-1}, T_{a}(A(x)) \cdot c_{A S A^{-1}}(a)^{-1}\right] .
$$

Vamos mostrar que $T_{[A, a]}$ é um automorfismo de $\left[K^{*}, L\right]$. De 2.28 temos

$$
T_{[A, a]}([S, x][V, y])=\left[A V S A^{-1}, T_{a} A(V(x) y) \cdot c_{A V S A^{-1}}(a)^{-1}\right]
$$

e $T_{[A, a]}([S, x]) T_{[A, a]}([V, y])=\left[A V S A^{-1}, w\right]$, onde

$$
w=A V A^{-1}\left(T_{a} A(x) c_{A S A^{-1}}\left(a^{-1}\right)\right) T_{a} A(y) c_{A V A^{-1}}(a)^{-1} .
$$

Agora notemos que

$$
\begin{aligned}
w & =T_{a} A V(x) \cdot T_{a} A(y) \cdot A V A^{-1} \cdot\left(c_{A S A^{-1}}\left(a^{-1}\right)\right) \cdot c_{A V A^{-1}}(a)^{-1} \\
& =T_{a} A(V(x) y) \cdot\left(A V A^{-1}\left(c_{A S A^{-1}}(a)^{-1}\right) \cdot c_{A V A^{-1}}(a)^{-1}\right) .
\end{aligned}
$$


Portanto, $T_{[A, a]}$ será um automorfismo de $\left[K^{*}, L\right]$ se e somente se valer

$$
A V A^{-1}\left(c_{A S A^{-1}}(a)^{-1}\right) \cdot c_{A V A^{-1}}(a)^{-1}=c_{A V S A^{-1}}(a)^{-1} .
$$

É fato que

$$
\begin{aligned}
A V S A^{-1}(a) & =A V A^{-1}\left(A S A^{-1}(a)\right) \\
& =A V A^{-1}\left(a \cdot c_{A S A^{-1}}\right)(a) \\
& =A V A^{-1}(a) A V A^{-1}\left(c_{A S A^{-1}}(a)\right) \\
& =a \cdot c_{A V A^{-1}}(a) A V A^{-1}\left(c_{A S A^{-1}}(a)\right) .
\end{aligned}
$$

Por outro lado,

$$
A V S A^{-1}(a)=a \cdot c_{A V S A^{-1}}(a)
$$

Então de 2.32 e 2.33 temos que vale a identidade 2.31. Logo $T_{[A, a]}$ é um automorfismo de $\left[K^{*}, L\right]$. Concluimos, então, que $\left[K^{*}, L\right]$ é um A-loop, como queríamos demonstrar.

\subsection{Construção e Exemplos de $A$-Loops}

Nessa seção apresentaremos uma nova maneira de se construir novos $A$-loops a partir de $A$-loops dados.

Sejam $L$ um loop, S e T automorfismos de $L$. Considere o conjunto $Q$ formado pelos pares $\left[A^{i}, x\right]$ onde $i=0,1$ e $x \in L$. Em $Q$, defina a seguinte multiplicação:

$$
\begin{gathered}
{[1, x][1, y]=[1, x y] \quad[1, x][A, y]=[A, S(x) y]} \\
{[A, x][1, y]=[A, x y] \quad[A, x][A, y]=[1, x T(y)]}
\end{gathered}
$$

Vamos mostrar que com esta multiplicação, $Q$ admite estrutura de loop. Para isso, primeiramente, mostremos que $Q$ é um quase-grupo. Considere a equação $\left[A^{i}, a\right]\left[A^{j}, x\right]=\left[A^{k}, b\right]$, com incógnita $\left[A^{j}, x\right]$ e onde $\left[A^{i}, a\right],\left[A^{k}, b\right] \in Q$.

1. Se $i=k=1$, faça $j=0$ e tome $x$ a única solução da equação $a x=b$ em $L$. A unicidade de $x$ nos garante a unicidade da solução $[1, x]$. 
2. Se $i=1$ e $k=0$, tome $j=1$ e $x$ a única solução da equação $a T(x)=b$. A unicidade de $[A, x]$ segue do mesmo argumento do ítem 1.

3. Para o caso de $i=0$ e $k=1$, tome $j=1$ e $x$ a única solução de $S(a) x=b$.

4. Finalmente para $i=k=0$, tome $j=0$ e $x$ a única solução de $a x=b$.

Para as equações $\left[A^{j}, x\right]\left[A^{i}, a\right]=\left[A^{k}, b\right]$ onde $i, k=0,1$ usamos raciocínio análogo ao usado acima. Assim temos que $Q$ éum quase-grupo. Para mostrar que $Q$ é um loop, vamos mostrar que $[1,1]$ é elemento neutro de $Q$. De fato,

$$
\begin{gathered}
{[1,1][1, x]=[1, x] \quad[1, x][1,1]=[1, x]} \\
{[1,1][A, x]=[A, S(1) x]=[A, x] \quad[A, x][1,1]=[A, x]}
\end{gathered}
$$

para qualquer $x \in L$. Logo temos que $Q$ é um loop.

Bruck e Paige, em [BP], mostraram o seguinte lema, do qual faremos uso na demosntração do próximo teorema.

Lema 2.5.1. Sejam $L$ um loop, $S, T$ automorfismos de $L$ tais que $T(x)=x^{-1}$ para todo $x \in \mathcal{Z}(L)$. Considere o loop $Q$ como definido acima, e $M: Q \longrightarrow Q$ definida por

$$
M([1, x])=[1, \alpha(x)] \quad M([A, x])=[A, \beta(x)]
$$

onde $\alpha, \beta$ são permutações de $L$. Então $M$ será um automorfismo de $Q$ se e somente se $\alpha$ for um automorfismo de $L$ que comuta com $S$ e $T$ e $\beta(x)=c \alpha(x)$ onde $c \in \mathcal{Z}(L)$.

Teorema 2.5.2. Seja $G$ um grupo abeliano e $S$ o automorfismo de $G$ dado por $S(x)=$ $x^{-1}$ para todo $x \in G$. Considere o loop $L=\left\{\left[S^{i}, x\right]: i=0,1 \quad x \in G\right\}$ sob a seguinte multiplicação

$$
\begin{array}{cc}
{[1, x][1, y]=[1, x y]} & {[1, x][S, y]=[A, S(x) y]} \\
{[S, x][1, y]=[A, x y]} & {[S, x][S, y]=[1, x S(y)] .}
\end{array}
$$

Então L é um A-loop. 
Demonstração. Vamos aqui usar o lema 2.5.1. Para isso vamos mostrar que os geradores de $\mathcal{I}(L)$, satisfazem as condições da aplicação $M$ desse lema. Tal demonstração será feita para apenas um gerador já que para os outros geradores o argumento é inteiramente análogo. Sejam $[S, a],[S, b] \in L$ e faça $R_{([S, a],[S, b])}([1, x])=[Z, z]$. Assim temos que

$$
\begin{gathered}
R_{[S, a][S, b]}^{-1} R_{[S, b]} R_{[S, a]}([1, x])=[Z, z], \\
R_{[S, b]([1, x][S, a])}=[Z, z]([S, a][S, b]), \\
{\left[S, x^{-1} a\right][S, b]=[Z, z]\left[1 . a b^{-1}\right],} \\
{[Z, z]\left[1, a b^{-1}\right]=\left[1,\left(x^{-1} a\right) b^{-1}\right],}
\end{gathered}
$$

o que implica que $Z=1$ e $z=R_{\left(a, b^{-1}\right)} S(x)$, isto é,

$$
R_{([S, a],[S, b])}([1, x])=\left[1, R_{\left(a, b^{-1}\right)} S(x)\right] .
$$

Da mesma forma, obtemos

$$
R_{([S, a],[S, b])}([S, x])=\left[S, R_{b^{2}} R_{(a, b)} S(x)\right] .
$$

Então aplicando o lema 2.5.1 para a aplicação $R_{([S, a],[S, b])}$, temos que esta é um automorfismo de L.

Bruck e Paige em [BP], mostraram um resultado mais geral para o teorema 2.5.2. Na verdade esse resultado vale se $G$ for um $A$-loop, $S$ um automorfismo de centro de $L$ e $T=S^{-1}$. O próximo resultado nos fala de construções de $A$-loops através de extensões centrais.

Teorema 2.5.3. Sejam $Z$ um grupo abeliano e $K$ um A-loop. Para cada par de elementos $(p, q)$ de $K$ determine um elemento $z(p, q) \in Z$, satisfazendo $z(p, 1)=$ $z(1, p)=1$ (os elementos neutros de $Z$ e $K$ serão denotados por 1 ) e respeitando as segintes restrições:

$$
F(p, s, t) F(q, s, t) z\left(R_{(s, t)}(p), R_{(s, t)}(q)\right)=F(p q, s, t) z(p, q)
$$


para todos $p, q, s, t \in K$, onde, $F(p, s, t)=z\left(R_{(s, t)}(p), s t\right)^{-1} z(s, t)^{-1} z(p s, t) z(p, s)$,

$$
G(p, s, t) G(q, s, t) z\left(L_{(s, t)}(p), L_{(s, t)}(q)\right)=G(p q, s, t) z(p, q)
$$

para todos $p, q, s, t \in K$, onde, $G(p, s, t)=z\left(t s, L_{(s, t)}(p)\right)^{-1} z(t, s)^{-1} z(t, s p) z(s, p) e$

$$
H(p, t) H(q, t) z\left(T_{t}(p), T_{t}(q)\right)=H(p q, t) z(p, q),
$$

para todos $p, q, t \in K$, onde, $H(p, t)=z\left(t, T_{t}(p)\right)^{-1} z(p, t)$.

Seja $G=K \ltimes_{z} Z$ o cinjunto dos pares $(p, x) \in K \times Z$ munido da seguinte operação binária:

$$
(p, x)(q, y)=(p q, z(p, q) x y) .
$$

Então $G$ é um A-loop. Além disso, o conjunto dos pares $(1, x), x \in Z$ é um subloop normal de $G$ contido no centro $\mathcal{Z}(G)$ e o loop quociente $G /(1, Z)$ é isomorfo a $K$.

Demonstração. As estruturas de grupo de $Z$ e de loop de $K$ fazem com que $G$ tenha estrutura de loop com elemento neutro $(1,1)$. Antes de mostrar que G é um A-loop vamos determinar como os geradores de $\mathcal{I}(G)$ agem em $G$. Sejam $(p, x),(q, y),(s, u) \in$ G. Faça $R_{((p, x),(q, y))}(s, u)=(t, v)$, então

$$
\begin{gathered}
R_{(p, x)(q, y)}^{-1} R_{(q, y)} R_{(p, x)}(s, u)=(t, v), \\
R_{(q, y)}((s, u)(p, x))=(t, v)((p, x)(q, y)), \\
(s p, z(s, p) u x)(q, y)=(t, v)(p q, z(p, q) x y), \\
(t(p q), z(t, p q) z(p, q) x y v)=((s p) q, z(s p, q) z(s, p) u x y),
\end{gathered}
$$

e assim $t=R_{(p, q)}(s)$ e $v=z(t, p q)^{-1} z(p, q)^{-1} z(s p, q) z(s, p) u$, ent $\tilde{a} o$

$$
R_{((p, x),(q, y))}(s, u)=\left(R_{(p, q)}(s), z\left(R_{(p, q)}(s), p q\right)^{-1} z(p, q)^{-1} z(s p, q) z(s, p) u\right)
$$

Procedendo da mesma forma, obtemos 


$$
\begin{aligned}
L_{((p, x),(q, y))}(s, u)= & \left(L_{(p, q)}(s), z\left(q p, L_{(p, q)}(s)\right)^{-1} z(q, p)^{-1} z(q, p s) z(s, p) u\right) \quad e \\
& T_{(p, x)}(s, u)=\left(T_{p}(s), z\left(p, T_{p}(s)\right)^{-1} z(s, p) u\right) .
\end{aligned}
$$

Agora é fácil ver que, pelas equações 2.39, 2.40 e 2.41, $R_{((p, x),(q, y))}, L_{((p, x),(q, y))}$ e $T_{(p, x)}$ serão automorfismos de $G$ se e somente se valerem as equações 2.36, 2.37 e 2.38, respectivamente. Portanto G é um A-loop.

Agora vamos mostrar que o conjunto $(1, Z)=\{(1, x): x \in Z\}$ é um subloop normal de $G$. Como $(1,1) \in(1, Z)$ temos que esse conjunto é não vazio. Sejam $(p, x),(q, y),(s, u) \in G$ tais que $(p, q)(q, y)=(s, u)$.

1. Se $(p, x),(q, y) \in(1, Z)$, temos que $p=q=1$ e assim $(s, u)=(1, x y) \in(1, Z)$,

2. $s e(p, x),(s, u) \in(1, Z)$, temos que $p=s=1$ e $\operatorname{assim}(q, y)=\left(1, x^{-1} u\right) \in(1, Z)$,

3. se $(s, u),(q, y) \in(1, Z)$, temos que $s=q=1$ e assim $(p, x)=\left(1, u y^{-1}\right) \in(1, Z)$. Assim $(1, Z)$ é um subloop de G. Pelas equações 2.39, 2.40 e 2.41, temos que $(1, Z)^{\varphi}=(1, Z)$ para todo $\varphi \in \mathcal{I}(L)$. Logo $(1, Z)$ é um subloop normal de $G$. Finalmente vamos mostrar que $G /(1, Z) \simeq K$. Para isso considere a sobrejeção $\Psi: G \longrightarrow K$ definida por $\Psi(p, x)=p$. Note que

$$
\Psi((p, x)(q, y))=\Psi(p q, z(p, q) x y)=p q=\Psi(p, x) \Psi(q, y)
$$

assim $\Psi$ é um homomorfiso sobrejetor entre os loops $G$ e $K$. Desde que $\Psi(p, x)=1$ se e somente se $(p, x) \in(1, Z)$ temos $\operatorname{ker}(\Psi)=(1, Z)$. Logo $G /(1, Z) \simeq K$. 


\section{Capítulo 3}

\section{A-Loops Comutativos Finitos: Estrutura}

Nesse capítulo estudaremos estrutura de um $A$-loop comutativo que, salvo meção contrária, denotaremos por $Q$. Para $A$-loops comutativos, têm-se muitos resultados interessantes dentre eles o Teorema da Ordem Ímpar que nos garante que todo $A$-loop comutativo finito de ordem ímpar é solúvel. Outro resultado bastante importante é o Teorema da Decomposição que afirma que todo $A$-loop comutativo finito é o produto direto de um $A$-loop de ordem ímpar por um $A$-loop de expoente 2. Encerraremos esse capítulo mostrando que valem os teoremas de Lagrange, Cauchy para $A$-loops comutativos finitos.

\subsection{Notações e Fatos Básicos}

Vamos agora estabelecer algumas noações e estabelecer alguns pequenos resultados que usaremos no decorrer do capítulo. Temos que $L_{x}: Q \longrightarrow Q$ é uma translação de $Q$ e, associada a essa translação, vamos definir uma divisão à esquerda por $x \backslash y=L_{x}^{-1}(y)$. Definiremos também as permutações de divisão $D_{x}: Q \longrightarrow Q$ por $D_{x}(y)=y \backslash x$. Temos que $D_{x}^{2}=i d_{Q}$ para todo $x \in Q$. Como $Q$ é comutativo temos 
que $x^{\rho}=x^{\lambda}$ que denotaremos por $x^{-1}$ e, assim, $x^{-1}=x \backslash 1=L_{x}^{-1}(1)$ e ainda podemos definir $J: Q \longrightarrow Q$ por $J(x)=x^{-1}$.

Notemos que se $u, v \in Q, u \backslash v=w$ se e somente se, $u w=v$. Desde que $L_{(x, y)}=L_{y x}^{-1} L_{y} L_{x} \in \mathcal{I}(L)$, temos que $L_{(x, y)}(v)=L_{(x, y)}(u w)=\left(L_{(x, y)}(u)\right)\left(L_{(x, y)}(w)\right)$, ou seja

$$
\left.L_{(x, y)}(u \backslash v)=\left(L_{(x, y)}(u)\right) \backslash\left(L_{(x, y)} v\right)\right)
$$

para quaisquer $x, y, u, v \in Q$. Também temos que $J L_{(x, y)}=L_{(x, y)} J$, para todos $x, y \in Q$.

Para $\varphi$ um automorfismo de $Q$, consideremos

$$
\operatorname{Fix}(\varphi)=\{x \in Q: \varphi(x)=x\}
$$

- Pelo lema 2.1.4, temos que Fix $(\varphi)$ é um subloop de $Q$ e que, portanto, se $x \in$ $F i x(\varphi)$, o subgrupo de $Q$, gerado por $x$, é de fato, subgrupo de Fix $(\varphi)$. Agora, tomando $x \in F i x(\varphi)$ e $y \in Q$ notemos que

$$
\varphi\left(L_{x}(y)\right)=\varphi(x y)=\varphi(x) \varphi(y)=x \varphi(y)=L_{x}(\varphi(y))
$$

e

$$
\varphi\left(D_{x}(y)\right)=\varphi(y \backslash x)=\varphi(y) \backslash \varphi(x)=\varphi(y) \backslash x=D_{x}(\varphi(y))
$$

Assim

$$
L_{x} \varphi=\varphi L_{x} \quad \text { e } \quad D_{x} \varphi=\varphi D_{x}
$$

para todo $x \in \operatorname{Fix}(\varphi)$.

Lema 3.1.1. Para quaisquer $x, y, z$ em um A-loop comutativo $Q, x \in F i x\left(L_{(y, z)}\right)$ se e somente se $L_{x} L_{z}(y)=L_{z} L_{x}(y)$ que é equivalente a $z \in F i x\left(L_{(y, x)}\right)$.

Demonstração. Temos que $x \in$ Fix $\left(L_{(y, z)}\right)$ se e somente se $L_{z y}^{-1} L_{z} L_{y}(x)=x$, isto é $z(y x)=(z y) x$. Como $Q$ é comutativo, esta última equação é o mesmo que $z(x y)=x(z y)$, ou seja $L_{x} L_{z}(y)=L_{z} L_{x}(y)$. Finalmente $z(x y)=x(z y)$ é equivalente a $L_{x y}(z)=L_{x} L_{y}(x)$, isto é, $z \in \operatorname{Fix}\left(L_{(y, x)}\right)$. 
Do teorema 2.1.7 temos que, em $Q$, vale que

$$
\begin{gathered}
L_{\left(y, x^{m}\right)}\left(x^{n}\right)=x^{n} ; \\
L_{x^{m}} L_{x^{n}}=L_{x^{n}} L_{x^{m}} \\
L_{x^{n}} L_{\left(y, x^{m}\right)}=L_{\left(y, x^{m}\right)} L_{x^{n}} \\
D_{x^{n}} L_{\left(y, x^{m}\right)}=L_{\left(y, x^{m}\right)} D_{x^{n}}
\end{gathered}
$$

para quaisquer $x, y \in Q$ e $n, m \in \mathbb{Z}$. Ainda temos que $Q$ associa potências, pelo teorema 2.1.5.

Lema 3.1.2. Para quaisquer $x, y \in Q$, valem as seguintes propriedades:

1. $L_{(y, x)}\left(y^{n}\right)=(x y \backslash x)^{-n}$, para todo $n \in \mathbb{Z}$

2. $x y^{2}=(x y)(x y \backslash x)^{-1}$.

Demonstração. Temos que $L_{(y, x)}\left(y^{-n}\right)=L_{(y, x)}\left(\left(y^{-1}\right)^{n}\right)=\left(L_{(y, x)}\left(y^{-1}\right)\right)^{n}$ pois $L_{(y, x)}$ é um automorfismo de $Q$. Assim $L_{(y, x)}\left(y^{-n}\right)=\left(L_{x y}^{-1} L_{x} L_{y}\left(y^{-1}\right)\right)^{n}=\left(L_{x y}^{-1}(x)\right)^{n}=$ $(x y \backslash x)^{n}$. Substituindo $n$ por $-n$ obtemos a identidade 1.

Temos que $L_{(y, x)}(y)=L_{x y}^{-1} L_{x} L_{y}(y)=L_{x y}^{-1}\left(y^{2} x\right)=(x y) \backslash\left(x y^{2}\right)$. Do item 1, temos que $(x y) \backslash\left(x y^{2}\right)=(x y \backslash x)^{-1}$, que nos dá o ítem 2 .

Pelo teorema 2.1.7, temos que todo $A$-loop comutativo $Q$, é um $P I$-loop, no sentido da definição dada no capítulo 1 . Um loop $L$ é dito ter a propriedade automórfica do inverso $(A I P)$ e se todos os seus elementos possuirem inverso bilateral se, para todos $x, y \in L$

$$
(x y)^{-1}=x^{-1} y^{-1} \quad \text { ou, equivalentemente } \quad L_{x} J=J L_{x^{-1}} .
$$

Lema 3.1.3. Todo A-loop comutativo tem AIP. 
Demonstração. Temos que $L_{x} L_{x^{-1}}=L_{\left(x^{-1}, x\right)}$ é um automorfismo de $Q$. De 3.4 temos que

$$
\begin{gathered}
J L_{x^{-1}} L_{x}(y)=J L_{x} L_{x^{-1}}(y)=L_{x} L_{x^{-1}}\left(y^{-1}\right)=L_{x}\left(x^{-1} y^{-1}\right)=L_{x} L_{y^{-1}}\left(x^{-1}\right)= \\
=\left[L_{x} L_{y}\right]\left[L_{y}^{-1} L_{y^{-1}}\right]\left(x^{-1}\right)=L_{x y} L_{x y}^{-1} L_{x} L_{y} L_{y^{-1}} L_{y}^{-1}\left(x^{-1}\right)=L_{x y}\left[L_{(y, x)} L_{y^{-1}}\right] L_{y}^{-1}\left(x^{-1}\right)= \\
=L_{x y} L_{L_{(y, x)}\left(y^{-1}\right)} L_{(y, x)} L_{y}^{-1}\left(x^{-1}\right)
\end{gathered}
$$

. Pelo ítem 1 do lema 3.1.2 temos

$$
\begin{gathered}
L_{x y}\left[L_{L_{(y, x)}\left(y^{-1}\right)} L_{(y, x)} L_{y}^{-1}\left(x^{-1}\right)\right]=L_{x y}\left[(x y)^{-1}(x y \backslash x)\right]= \\
=L_{x y} L_{(x y)}^{-1} L_{(x y)^{-1}}(x)=L_{(x y)^{-1}}(x)=L_{x}\left(x y^{-1}\right)=L_{x} J L_{x}(y) .
\end{gathered}
$$

Dai temos que, $L_{x^{-1}} J=J L_{x}$ para todo $x \in L$

Lema 3.1.4. Num A-loop comutativo $Q$, para quaisquer $x, y \in Q$, as seguintes $i d e n$ tidades se verificam:

1. $L_{(x, y)}=L_{\left(x^{-1}, y^{-1}\right)}$;

2. $L_{(x, y)}=L_{y} L_{x} L_{x^{-1} \backslash y}^{-1}$;

3. $L_{(x, y)}=L_{x} L_{x^{-1} \backslash y}^{-1} L_{y}$;

4. $L_{(x \backslash y, x)}=L_{\left((y \backslash x)^{-1}, x\right)}$;

5. $L_{(x \backslash y)^{-1} \backslash x}^{-1} L_{x \backslash y}=L_{y}^{-1} L_{(y \backslash x)^{-1}}$.

\section{Demonstração.}

1. Seja $z \in Q$. Temos que

$$
\begin{gathered}
\left(L_{(x, y)}(z)\right)^{-1}=J\left(L_{(x, y)}(z)\right)=L_{(x, y)} J(z)=L_{y x}^{-1} L_{y} L_{x} J(z)=J L_{(y x)^{-1}}^{-1} L_{y^{-1}} L_{x^{-1}}(z)= \\
=J\left(L_{\left(x^{-1}, y^{-1}\right)}(z)\right)=\left(L_{\left(x^{-1}, y^{-1}\right)}(z)\right)^{-1} .
\end{gathered}
$$

Ou seja, $L_{(x, y)}=L_{\left(x^{-1}, y^{-1}\right)}$. 
2. Temos que

$$
L_{y} L_{x} L_{x^{-1} \backslash y}^{-1}=L_{x y} L_{(x, y)} L_{x^{-1} \backslash y}^{-1}=L_{x y} L_{(x, y)} L_{x^{-1} \backslash y}^{-1} L_{(x, y)}^{-1} L_{(x, y)} .
$$

Assim

$$
L_{y} L_{x} L_{x^{-1} \backslash y}^{-1}=L_{x y} L_{L_{(x, y)}\left(x^{-1} \backslash y\right)}^{-1} L_{(x, y)}=L_{x y} L_{L_{\left(x^{-1}, y^{-1}\right)}^{-1}\left(x^{-1} \backslash y\right)} L_{(x, y)}=L_{(x, y)},
$$

pois $L_{\left(x^{-1}, y^{-1}\right)}\left(x^{-1} \backslash y\right)=y x$.

3. Como $L_{(x, y)} L_{y}^{-1}=L_{y}^{-1} L_{(x, y)}=L_{x} L_{x^{-1} \backslash y}^{-1}$, temos que $L_{(x, y)}=L_{x} L_{x^{-1} \backslash y}^{-1} L_{y}$.

4. Como $x(y \backslash x)=y$ e $L_{(x \backslash y, x)}(x) x$, temos

$$
\begin{gathered}
L_{(x \backslash y, x)}=L_{(x \backslash y, x)}^{2} L_{(x \backslash y, x)}^{-1}=L_{(x \backslash y, x)} L_{(x \backslash y) x}^{-1} L_{x} L_{x \backslash y} L_{(x \backslash y, x)} L_{(x \backslash y, x)}^{-1}= \\
=L_{L_{(x \backslash y, x)}((x \backslash y) x)}^{-1} L_{L_{(x \backslash y, x)}(x)} L_{L_{(x \backslash y, x)}(y \backslash y)} L_{(x \backslash y, x)} L_{(x \backslash y, x)}^{-1}= \\
=L_{\left(L_{(x \backslash y, x)}(x \backslash y), L_{(x \backslash y, x)}(x)\right)} L_{(x \backslash y, x)} L_{(x \backslash y, x)}^{-1}=L_{\left((y \backslash x)^{-1}, x\right)} .
\end{gathered}
$$

5. Aplicando o ítem 2 na identidade $L_{(x \backslash y, x)}=L_{\left((y \backslash x)^{-1}, x\right)}$, temos que

$$
L_{x \backslash y} L_{(x \backslash y)^{-1} \backslash x}^{-1}=L_{(y \backslash x)^{-1}} L_{(y \backslash x) \backslash x}^{-1} .
$$

Notemos que $(y \backslash x) \backslash x=w \backslash x=u$ onde $y w=x$ e $w u=x$, então pela lei do cancelamento $u=y$. Então

$$
L_{x \backslash y} L_{(x \backslash y)^{-1} \backslash x}=L_{(y \backslash x)^{-1}} L_{y}^{-1} .
$$

Lema 3.1.5. Para todos $x, y$ em um A-loop comutativo $Q$, valem

1. $D_{x^{2}}=D_{x} J D_{x}$;

2. $x^{2}=D_{x}(y) D_{x}\left(y^{-1}\right)$;

3. $x=D_{x^{-1}}\left(y^{-1}\right) D_{x^{2}}(y)$. 


\section{Demonstração.}

1. Para qualquer que seja $y \in Q$, temos

$$
D_{x^{2}}(y)=L_{y}^{-1}\left(x^{2}\right)=L_{y}^{-1} L_{x}(x)=L_{(x \backslash y, x)} L_{x \backslash y}^{-1}(x),
$$

então

$$
\begin{gathered}
D_{x^{2}}(y)=L_{L_{(x \backslash y, x)}^{-1}(x \backslash y)} L_{(x \backslash y, x)}(x)=L_{((x(x \backslash y)) \backslash x)^{-1}}^{-1} L_{(x \backslash y, x)}(x)= \\
=L_{(y \backslash x)^{-1}}^{-1} L_{(x \backslash y, x)}(x)=L_{(y \backslash x)^{-1}}^{-1}(x)=(y \backslash x)^{-1} \backslash x=D_{x}\left((y \backslash x)^{-1}\right)=D_{x} J D_{x}(y) .
\end{gathered}
$$

$A \operatorname{sim} D_{x^{2}}=D-x J D_{x}$.

2. De $D_{x^{2}}=D_{x} J D_{x}$, temos que $D_{x} J=D_{x^{2}} D_{x}$ pois $D_{x}^{2}=i d_{Q}$. Então $D_{x}\left(y^{-1}\right)=D_{x^{2}} D_{x}(y)=L_{D_{x}(y)}^{-1}\left(x^{2}\right)$, ou seja $x^{2}=D_{x}(y) D_{x}\left(y^{-1}\right)$.

3. Temos que $D_{x^{2}}(y)=D_{x} J D_{x}(y)=L_{J D_{x}(y)}^{-1}(x)$. Como $Q$, tem AIP, segue que $x=J D_{x}(y) D_{x^{2}}(y)=D_{x^{-1}}\left(y^{-1}\right) D_{x^{2}}(y)$.

\subsection{A-loops Comutativos Finitos de Ordem Ímpar}

O principal objetivo dessa seção é o Teorema da Ordem Ímpar que nos diz que todo $A$-loop comutativo finito de ordem ímpar é solúvel.

Definição 3.2.1. Um loop L será dito unicamente 2-divisível se a aplicação $x \mapsto x^{2}$ for uma permutação de $L$.

Lema 3.2.2. Um loop finito, comutativo e que associa potências $Q$ será unicamente 2-divisível se e somente se tiver ordem ímpar.

Demonstração. Vamos supor $Q$ unicamente 2-divisível. Assim, $J(x)=x^{-1}=x$ se e somente se $x=1$. Daí temos que o conjunto $Q \backslash\{1\}$ tem ordem par, ou seja $Q$ tem ordem impar. 
Reciprocamente, suponha que $Q$ tenha ordem impar, e fixe um elemento $c \in Q$. Considere os conjuntos

$$
U=\{(x, y) \in Q \times Q: x y=c \quad e \quad x \neq y\}
$$

$e$

$$
V=\{(x, y) \in Q \times Q: x y=c\}
$$

Temos que $|V|=|Q|$ e, por comutatividade, $U$ tem ordem par. Portanto $V \backslash U$ tem ordem ímpar e, assim, é não vazio. Logo a aplicação $x \mapsto x^{2}$ é sobrejetiva e então, como $Q$ é finito, é bijetiva.

Definição 3.2.3. Um subgrupo torcido de um grupo $G$ é um subconjunto $T$ que satisfaz:

1. $1 \in T$;

2. $a^{-1} \in T$ sempre que $a \in T$;

3. aba $\in T$ sempre que $a, b \in T$

Um subgrupo torcido $T$ de um grupo $G$ será unicamente 2-divisível se a restrição da aplicação $x \mapsto x^{2}$ for uma bijeção de $T$ (Note que de $a^{2}=a 1 a \in T$ sempre que $a \in T$ ). Para $a, b$ em um unicamente 2-divisível subgrupo torcido $T$, podemos definir $a \circ b=\left(a b^{2} a\right)^{\frac{1}{2}}$, onde $x^{\frac{1}{2}}$ denota a única raiz quadrada de $x \in T$. Temos que $(T, \circ)$ é um loop de Bol (à esquerda), isto é

$$
x \circ(y \circ(x \circ z))=(x \circ(y \circ x)) \circ z,
$$

para quaisquer $x, y, z \in T$. Além disso, $(T, \circ)$ tem a propriedade $(A I P)$. Em outras palavras, $(T, \circ)$ é um loop de Bruck à esquerda.

Para um loop de Bol $Q$, temos o conjunto $L_{Q}=\left\{L_{x}: x \in Q\right\}$ é um subgrupo torcido de $\mathcal{M}(L)$, o grupo das multiplicações de $Q$. No caso de $Q$ ser unicamente 2-divisível $L_{Q}$ tem estrutura de loop de Bruck à esquerda. Essa estrutura pode ser 
passada isomorficamente para $Q$ e assim o conjunto $Q$ admite duas estruturas de loop: a estrutura de loop de Bol original e a estrutura de loop de Bruck. Um fato já conhecido é que potências nessas duas estruturas coincidem, assim é possível mostrar alguns resultados para o loop de Bol $Q$ usando a estrutura de loop de Bruck do conjunto Q. Glauberman em [Gl-02] usou esse raciocínio para loops de Moufang. Nesse trabalho, não é de interesse o estudo de loops de Moufang tampouco loops de Bol, entretando usaremos essa idéia para mostrar alguns resultados para $A$-loops comutativos finitos.

Para cada $x$ em um $A$-loop comutativo $Q$, definimos

$$
P_{x}=L_{x} L_{x^{-1}}^{-1}=L_{x^{-1}}^{-1} L_{x}
$$

Temos que $P_{Q}=\left\{P_{x}: x \in Q\right\} \subset \mathcal{M}(Q)$ satisfaz as condições 1 e 2 da definição de subgrupo torcido, pois $i d_{Q}=P_{1}$ e, como $P_{x} P_{x^{-1}}=i d_{Q}, P_{x}^{-1}=P_{x^{-1}}$.

Lema 3.2.4. Sejam $Q$ um A-loop comutativo e $x, y \in Q$. Então:

1. $P_{x y}\left(x^{-1}\right)=x y^{2}$;

2. $P_{x y} L_{x^{-1}}=L_{x} P_{y}$.

Demonstração. Pelo ítem 2 do lema 3.1.2 temos que

$$
x y^{2}=(x y)(x y \backslash x)^{-1}=(x y) J(x y \backslash x)=(x y) J\left(L_{x y}^{-1}(x)\right),
$$

e, como $Q$ tem AIP segue que

$$
(x y) J\left(L_{x y}^{-1}(x)\right)=L_{x y} J L_{x y}^{-1}(x)=L_{x y} L_{(x y)^{-1}}^{-1} J(x)=P_{x y}\left(x^{-1}\right),
$$

o que prova 1. Para provar 2, temos

$$
P_{x y} L_{x^{-1}}=L_{x y} L(x y)^{-1^{-1}} L_{x^{-1}}=L_{x y} L_{x^{-1} y^{-1}}^{-1} L_{x^{-1}}=L_{x y} L_{x^{-1} y^{-1}}^{-1} L_{x^{-1}} L_{y^{-1}} L_{y^{-1}}
$$

ou seja,

$$
P_{x y} L_{x^{-1}}=L_{x y} L_{\left(y^{-1}, x^{-1}\right)} L_{y^{-1}}=L_{x y} L_{(y, x)} L_{y^{-1}}=L_{x} L_{y} L_{y^{-1}}^{-1}=L_{x} P_{y}
$$


Lema 3.2.5. Para quaisquer $x, y \in Q, P_{x} P_{y} P_{x}=P_{P_{x}(y)}$. Em particular, $P_{Q}$ é um subgrupo torcido de $\mathcal{M}(Q)$.

Demonstração. Primeiramente notemos que

$$
x^{-1} \cdot P_{x}(y)=L_{x^{-1}} P_{x}(y)=L_{x^{-1}} L_{x^{-1}}^{-1} L_{x}(y)=L_{x}(y)=x y .
$$

Pelo ítem 2 do lema anterior e esta última observação, temos que

$$
P_{x} P_{y} P_{x}=L_{x^{-1}}^{-1} L_{x} P_{y} P_{x}=L_{x^{-1}}^{-1} P_{x y} L_{x^{-1}} P_{x}=L_{x^{-1}}^{-1} P_{x y} L_{x}=L_{x^{-1}}^{-1} P_{x^{-1} P_{x}(y)} L_{x},
$$

e assim, $P_{x} P_{y} P_{x}=L_{x^{-1}}^{-1} L_{x^{-1}} P_{P_{x}(y)}=P_{P_{x}(y)}$.

Lema 3.2.6. Para todo $x$ em um A-loop comutativo $Q$ e para todo $n$ em $\mathbb{Z}$,

$$
P_{x}^{n}=P_{x^{n}}
$$

Demonstração. Vamos proceder por indução sobre $n \in \mathbb{N}$. Para $n=0$ ou $n=1$ não há nada a fazer.

Se $P_{x}^{n}=P_{x^{n}}$ vale para algum $n$, temos que $P_{x}^{n+2}=P_{x} P_{x}^{n} P_{x}=P_{x} P_{x^{n}} P_{x}=$ $P_{P_{x}\left(x^{n}\right)}=P_{x}^{n+2}$, desde que $P_{x}\left(x^{n}\right)=x^{n+2}$. Por indução, temos que $P_{x}^{n}=P_{x^{n}}$ vale para todo $n \in \mathbb{N}$. Como $P_{x}^{-1}=P_{x^{-1}}$, segue que $P_{x}^{n}=P_{x^{n}}$ vale para todo $n \in \mathbb{Z}$.

Assumindo que $Q$ seja um $A$-loop comutativo unicamente 2-divisível, pelo último lema temos que $P_{Q}$ é um subgrupo torcido de $\mathcal{M}(Q)$ que também é unicamente 2-divisível. Para $x, y \in Q$, vamos definir

$$
P_{x} \circ P_{y}=\left(P_{x} P_{y}^{2} P_{x}\right)^{\frac{1}{2}}=\left(P_{x} P_{y^{2}} P_{x}\right)^{\frac{1}{2}}=\left(P_{P_{x}\left(y^{2}\right)}\right)^{\frac{1}{2}}=P_{\left(P_{x}\left(y^{2}\right)\right)^{\frac{1}{2}}} .
$$

Assim, está definida em $Q$ uma nova operação binária, a qual também denotaremos por $\circ$.

$$
x \circ y=\left(P_{x}\left(y^{2}\right)\right)^{\frac{1}{2}}=\left(x^{-1} \backslash x y^{2}\right)^{\frac{1}{2}} .
$$


Lema 3.2.7. Para um A-loop comutativo unicamente 2-divsível $Q,(Q, \circ)$ é um loop de Bruck. Além disso, as potências em $Q$ coincidem com as potências em $(Q, \circ)$.

Demonstração. Vimos que $P_{Q}$ é um subgrupo torcido de $\mathcal{M}(Q)$ e como $P_{x}^{2}=P_{x^{2}}$ para todo $x \in Q$ e $Q$ é unicamente 2-divisível, temos que $P_{Q}$ também é unicamente 2-divisivel. Em $P_{Q}$, definindo

$$
P_{x} \circ P_{y}=P_{\left(P_{x}\left(y^{2}\right)\right)^{\frac{1}{2}}}
$$

temos que $P_{Q}$ é um loop de Bruck. A aplicação $x \mapsto P_{x}$ é, claramente, um homomorfismo sobrejetor de $(Q, \circ)$ em $\left(P_{Q}, \circ\right)$. Se $P_{x}=i d_{Q}$, temos $x^{2}=P_{x}(1)=1$ o que implica que $x=1$, pois $Q$ é unicamente 2-divisivel. Portanto $(Q, \circ)$ é um loop de Bruck. Para mostrar que as potências de $(Q, \circ)$ coincidem com as potências de $Q$, vamos usar o argumento de indução sobre n. Como $Q$ associa potências temos que

$$
x \circ x=\left(x^{-1} \backslash x^{3}\right)^{\frac{1}{2}}=\left(x^{4}\right)^{\frac{1}{2}}=x^{2} .
$$

Agora assuma que $x \circ x^{n-1}=x^{n}$, então

$$
x \circ x^{n}=\left(x^{-1} \backslash x x^{2 n}\right)^{\frac{1}{2}}=\left(x^{-1} \backslash x^{2 n+1}\right)^{\frac{1}{2}}=\left(x^{2 n+2}\right)^{\frac{1}{2}}=x^{n+1},
$$

como queríamos demonstrar.

Definição 3.2.8. Dizemos que o loop $(H, \star)$ é um B-loop se:

a) $(H, \star)$ associa potências;

b) $(H, \star)$ satisfaz a identidade 3.8;

c) $(H, \star)$ tem $(A I P)$;

d) Todo elemento de $H$ tem ordem finita impar.

Temos que se $Q$ for um $A$-loop comutativo, finito e unicamente 2-divisível, $(Q$, o) será um $B$-loop. 
Proposição 3.2.9. Seja $A \leq B$ subloops de um $A$-loop comutativo finito $Q$, de ordem impar. Entã $|A|$ divide $|B|$. Em particular, a ordem de qualquer elemento de $Q$, divide a ordem de $Q$.

Demonstração. Glauberman em [Gl-01], provou o mesmo resultado para B-loops finitos. O que vamos fazer é mostrar que os subloops $A$ e $B$ de $Q$, nos dão subloops $(A, \circ)$ e $(B, \circ)$ de $(Q, \circ)$. Temos que A é não vazio e unicamente 2-divisível. Como $A$ é finito, basta mostrarmos que A é fechado para a operação o. De fato, para $x, y \in A$, temos

$$
x \circ y=\left(x^{-1} \backslash x y^{2}\right)^{\frac{1}{2}}=\left(L_{x^{-1}}^{-1}\left(x y^{2}\right)\right)^{\frac{1}{2}} \in A .
$$

Logo $(A, \circ)$ é um subloop de $(Q, \circ)$. Do mesmo modo provamos que $(B, \circ)$ é um subloop de $(Q, \circ)$. Assim vale o resultado para $Q$.

Proposição 3.2.10 (Cauchy). Seja $Q$, um A-loop comutativo, finito de ordem impar. Se um primo $p$ divide $|Q|$, então $Q$ possui um elemento de oredm $p$.

Demonstração. Como Glauberman em [Gl-01] provou esse resultado para B-loops finitos, temos que essa proposição vale para $(Q, \circ)$. Desde que potências em $Q$ e em $(Q, \circ)$ coincidem, segue o resultado para o loop $Q$.

Para um conjunto de números primos positivos $\pi$, um número inteiro positivo $n$ é dito um $\pi$-número, se $n=1$ ou se $n$ se escreve como produto de primos em $\pi$. Para cada inteiro positivo $n, n_{\pi}$ denotará o maior $\pi$-número que divide $n$.

Definição 3.2.11. Para um conjunto de números primos positivos $\pi$, um subloop $K$ de um loop finito que associa potências $L$, é chamado $\pi$-subloop de Hall se $|K|=|L|_{\pi}$. Em particular, se $\pi=\{p\}$, dizemos que $K$ é um p-subloop de Sylow.

Jedlicka, Kinyon e Vojtechovsky suspeitam que para um $A$-loop comutativo finito de ordem ímpar, existam subloops de Hall e Sylow, mas não há prova para esse fato. 
Lema 3.2.12. Toda aplicação interna de um A-loop comutativo unicamente 2-divisível $Q$, age como um automorfismo para $(Q, \circ)$.

Demonstração. Vamos mostrar que os geradores de $\mathcal{I}(L)$ são automorfismos de $(Q, \circ)$. Para quaisquer $x, y, a, b \in Q$, temos

$$
\begin{aligned}
L_{(x, y)}(a \circ b) & =L_{(x, y)}\left[\left(a^{-1} \backslash a b^{2}\right)^{\frac{1}{2}}\right] \\
& =\left[L_{(x, y)}\left(a^{-1} \backslash a b^{2}\right)\right]^{\frac{1}{2}} \\
& =\left[L_{(x, y)}(a)^{-1} \backslash\left(L_{(x, y)}(a) L_{(x, y)}(b)^{2}\right)\right]^{\frac{1}{2}} \\
& =L_{(x, y)}(a) \circ L_{(x, y)}(b) .
\end{aligned}
$$

Lema 3.2.13. Seja $Q$, um A-loop comutativo finito de ordem ímpar. Um subloop $K$ de $(Q, \circ)$ é um subloop de $Q$ se e somente se $\varphi(K)=K$ para cada $\varphi \in X=$ $\mathcal{I}(Q) \cap\left\langle L_{x}: x \in K\right\rangle$

Demonstração. Claramente se $K \leq(Q, \circ)$ também for um subloop de $Q$, então $\varphi(K)=K$ para toda $\varphi \in X$. Reciprocamente vamos supor que, para qualquer $\varphi \in X$ temos $\varphi(K)=K$. Sejam $u, v \in K$, devemos mostrar que uv, $L_{u}^{-1}(v) \in K$. Como potências em $Q$ e em $(Q, \circ)$ coincidem, temos que $u^{-1}, v^{-1}, v^{\frac{1}{2}} \in K$. Assim

$$
\left(u \circ v^{\frac{1}{2}}\right)^{2}=L_{u^{-1}}^{-1} L_{u}(v)=L_{u^{-1}}^{-1} L_{u}^{-1} L_{u}^{2}(v)=L_{\left(u^{-1}, u\right)}^{-1} L_{u}^{2}(v) \in K
$$

Por hipótese $L_{u}^{2}(v) \in K$. Por indução, já que $L_{u}^{2} \in X$, temos que $L_{u}^{2 k}(v) \in K$, para todo inteiro $k \in \mathbb{Z}$.

Se $|u|=2 n+1$, temos $L_{u}^{2 n+1} \in \mathcal{I}(L)$, pois $L_{u}^{2 n+1}(1)=u^{2 n+1}=1$. Portanto,

$$
L_{u}^{2 n+1} L_{u}^{-2 n}(v)=u v \quad e \quad L_{u}^{2 n+1} L_{u}^{2(-n-1)}(v)=L_{u}^{-1}(v)
$$

estão em $K$. Logo $K$ é um subloop de $Q$.

Lema 3.2.14. Seja $Q$ um A-loop comutativo e assuma que a identidade

$$
P_{x}\left(y^{2}\right)=P_{y}\left(x^{2}\right)
$$


valha para todos $x, y \in Q$. Então para quaisquer $x, y \in Q$ temos

$$
P_{x}\left(y^{2}\right)=x^{2} y^{2}
$$

Corolário 3.2.15. Seja $Q$ um A-loop comutativo unicamente 2-divisível. Então $(Q, \circ)$ será comutativo se e somente se $(Q, \circ)$ for isomorfo a $Q$.

Demonstração. De fato, supondo $Q$ comutativo e unicamente 2-divisível, temos que se $(Q, \circ)$ for comutativo, a identidade 3.11 valerá para quaisquer $x, y \in Q$, e assim, 3.12 nos diz que a aplicação $x \mapsto x^{2}$ é um isomorfismo de $(Q, \circ)$ em $Q$. Claramente, se $(Q, \circ)$ for isomorfo a $Q$, aquele será comutativo.

Demonstração (Demonstração do Lema 3.2.14). Primeiramente, vamos mostrar que

$$
P_{x}\left(x y^{2}\right)=P_{y}(x)
$$

para quaisquer $x, y \in Q$. De fato, temos que

$$
P_{x}\left(x y^{2}\right)=L_{x} L_{x^{-1}}^{-1}\left(x y^{2}\right)=L_{x} P_{x}\left(y^{2}\right)=L_{x} P_{y}\left(x^{2}\right) .
$$

Desde que $x^{-1} \backslash x=x^{2}$, temos

$$
P_{x}\left(x y^{2}\right)=L_{x^{-1}} P_{x} P y P_{x}(1)=L_{x^{-1}} P_{P_{x}(y)}(1)=x^{-1}\left(P_{x}(y)\right)^{2}=P_{x^{-1} P_{x}(y)}(x),
$$

e como, $x^{-1} P_{x}(y)=x y$, temos que $P_{x}\left(x y^{2}\right)=P_{x y}(x)$.

Também afirmamos que

$$
P_{y^{2}}\left(x^{-1}\right)=L_{x} P_{y} P_{x^{-1}}\left(y^{2}\right) \quad \text { para todos } \quad x, y \in Q .
$$

Temos que

$$
\begin{aligned}
P_{y^{2}}\left(x^{-1}\right) & =P_{x \cdot x \backslash y^{2}}\left(x^{-1}\right)=x\left(x \backslash y^{2}\right)^{2}=L_{x}\left(\left(x \backslash y^{2}\right)^{2}\right) \\
& =L_{x} P_{y} P_{y^{-1}}\left(\left(x \backslash y^{2}\right)^{2}\right)=L_{x} P_{y} P_{x \backslash y^{2}}\left(y^{-2}\right) \\
& =L_{x} P_{y} L_{x \backslash y^{2}} L_{\left(x \backslash y^{2}\right)^{-1}}^{-1}\left(y^{-2}\right) \\
& =L_{x} P_{y}\left(\left(x \backslash y^{2}\right) \cdot\left(x \backslash y^{2}\right)^{-1} \backslash y^{-2}\right) \\
& =L_{x} P_{y}\left(\left(x \backslash y^{2}\right) \cdot\left(x^{-1} \backslash y^{-2}\right) \backslash y^{-2}\right) \\
& =L_{x} P_{y}\left(x^{-1}\left(x \backslash y^{2}\right)\right)=L_{x} P_{y} L_{x^{-1}} L_{x}^{-1}\left(y^{2}\right)=L_{x} P_{y} P_{x^{-1}}\left(y^{2}\right)
\end{aligned}
$$


Agora, faça

$$
\begin{aligned}
L_{x} P_{y} P_{x}\left(y^{2}\right) & =L_{x} P_{y}^{2}\left(x^{2}\right)=L_{x^{-1}} P_{x} P_{y^{2}}\left(x^{2}\right)=L_{x^{-1}} P_{x} P_{y^{2}} P_{x}(1) \\
& =L_{x^{-1}} P_{P_{x}\left(y^{2}\right)}=x^{-1}\left(P_{x}\left(y^{2}\right)\right)^{2}=P_{x^{-1} P_{x}\left(y^{2}\right)}(x) \\
& =P_{x y^{2}}(x)=P_{x y^{2}} P_{x y} P_{x^{-1} y^{-1}}(x)=P_{x y^{2}} P_{x y}\left(x^{-1} y^{-2}\right) \\
& =P_{x y^{2}} P_{x y^{2}\left(x y^{2} \backslash x y\right.}\left(\left(x y^{2}\right)^{-1}\right)=P_{x y^{2}}\left(\left(x y^{2}\right)\left(x y^{2} \backslash x y\right)^{2}\right) \\
& =P_{x y^{2}\left(x y^{2} \backslash x y\right)}\left(x y^{2}\right)=P_{x y}\left(x y^{2}\right)=P_{x y}^{2}\left(x^{-1}\right)=P_{(x y)^{2}}\left(x^{-1}\right) \\
& =L_{x} P_{x y} P_{x^{-1}}\left(x y^{2}\right) .
\end{aligned}
$$

De, $L_{x} P_{y} P_{x}\left(y^{2}\right)=L_{x} P_{x y} P_{x^{-1}}\left(x y^{2}\right)$, temos que

$$
P_{y} P_{x}\left(y^{2}\right)=P_{x y} P_{x^{-1}}\left(x y^{2}\right)=P x y P_{x^{-1}} P_{x y}(1)=P_{P_{x y}\left(x^{-1}\right)}(1) P_{x y^{2}}(1)=\left(x y^{2}\right)^{2},
$$

assim,

$$
P_{x}\left(y^{2}\right)=P_{y^{-1}}\left(\left(x y^{2}\right)^{2}\right)=P_{y^{2} x}\left(y^{-2}\right)=y^{2} x^{2},
$$

o que conclui o lema.

Antes de ver o resultado mais importante dessa seção, vamos definir o que é um loop solúvel.

Definição 3.2.16. Um loop $L$ é dito solúvel se existirem subloops $N_{i} \quad(i=0, \ldots, r)$ tais que

$$
\{1\}=N_{r} \leq N_{r-1} \leq \ldots \leq N_{1} \leq N_{0}=L,
$$

onde $N_{i} \triangleleft N_{i-1}$ para todo $i=1, \ldots, r$ e $N_{i-1} / N_{i}$ é um grupo abeliano.

Teorema 3.2.17. [Teorema da Oredem Ímpar] Todo A-loop comutativo e finito de ordem impar é solúvel.

Demonstração. Suponha que exista um A-loop comutativo, finito, de ordem ímpar, não solúvel. Seja $Q$ o contra-exemplo de menor ordem. Desde que subloops normais e quocientes de $Q$ também têm ordem ímpar, temos que $Q$ deve ser simples. 
Tome $N$ o subloop derivado de $(Q, \circ)$, isto é menor subloop normal de $(Q, \circ)$ tal que $(Q / N, \circ)$ é um grupo abeliano. Em [Gl-02] tem-se qualquer loop de Bruck finito de ordem impar é solúvel, donde tiramos que $N$ é um subloop próprio. Como $N$ é fixado por qualquer automorfismo de $(Q, \circ)$, pelo lema 3.2.12, temos que $N$ é fixado por qualquer elemento de $\mathcal{I}(L)$. Assim pelo lema 3.2.13, temos que $N$ é um subloop de $Q$. Além disso, $N \triangleleft Q$, pois $\varphi(N)=N$, para todo $\varphi \in \mathcal{I}(L)$.

Desde que $Q$ é simples, temos $N=1$ e assim $(Q, \circ)$ é um grupo abeliano. Assim, pelo corolário, 3.2.15, temos $Q$ um grupo abeliano, que contradiz a suposição de $Q$ ser não-solúvel. Logo, todo A-loop comutativo, finito e de ordem ímpar é solúvel.

\subsection{Quadrados e Loop Associado}

Durante essa seção, $Q$ denotará um $A$-loop comutativo.

Ao contrário de grupos abelianos e loops de Moufang comutativos, onde o produto de dois quadrados ainda é um quadrado, em $A$-loops comutativos, nem sempre vale $(x y)^{2}=x^{2} y^{2}$ para quaisquer $x, y$. Vamos, em $Q$, definir a seguinte operação binária: para quaisquer $x, y \in Q$ seja

$$
x * y=((x y \backslash x)(y x \backslash y))^{-1} .
$$

Desde que, em $Q$ vale a $(A I P)$ e, pelo lema 3.1.2, temos que a definição 3.15 pode ser reescrita como

$$
x * y=L_{(y, x)}(y) L_{(x, y)}(x)
$$

A definição 3.16 é motivada no seguinte teorema:

Teorema 3.3.1. Para quaisquer $x, y$ em um A-loop comutativo, temos

$$
x^{2} y^{2}=(x * y)^{2} \text {. }
$$

Para demonstrar o teorema 3.3.1, serão necessários alguns lemas. 
Lema 3.3.2. Para todos $x, y \in Q, x * y=x^{2}\left(x \backslash(x y \backslash x)^{-1}\right)$.

Demonstração. Como,

$$
L_{(x, y)}(x)=L_{y x}^{-1}\left(x^{2} y\right)=L_{y x}^{-1} L_{x^{2}} L_{x} L_{x}^{-1}(y)=L_{(y, x)} L_{y}^{-1} L_{x^{2}} L_{x}^{-1}(y),
$$

temos que

$$
\begin{aligned}
x * y & =L_{(y, x)}(y) L_{(x, y)}(x)=L_{(y, x)}(y)\left(L_{(y, x)} L_{y}^{-1} L_{x^{2}} L_{x}^{-1}(y)\right) \\
& =L_{(y, x)}\left(y \cdot L_{y}^{-1} L_{x^{2}} L_{x}^{-1}(y)\right)=L_{(y, x)} L_{x^{2}} L_{x}^{-1}(y) \\
& =L_{x^{2}} L_{x}^{-1} L_{(y, x)}(y)=L_{x^{2}} L_{x}^{-1}(x y \backslash x)^{-1} \\
& =x^{2}\left(x \backslash(x y \backslash x)^{-1}\right) .
\end{aligned}
$$

Na passagem 3.17 usamos o lema 3.1.2.

Lema 3.3.3. Para todos $x, y \in Q, x^{-1} \backslash(x y \backslash x)=y \backslash(y x \backslash y)^{-1}$.

Demonstração. Afirmamos que $x=L_{x y} L_{x^{-1}}\left(y \backslash(y x \backslash y)^{-1}\right)$. De fato,

$$
\begin{aligned}
L_{x y} L_{x^{-1}}\left(y \backslash(y x \backslash y)^{-1}\right) & =L_{x y} L_{x^{-1}} L_{y}^{-1}\left((y x \backslash y)^{-1}\right)=L_{x y} L_{x^{-1}} L_{x \backslash x y}^{-1}\left((y x \backslash y)^{-1}\right) \\
& =L_{\left(x^{-1}, x y\right)}\left((y x \backslash y)^{-1}\right)=\left[L_{\left(x^{-1}, x y\right)}(x y) \backslash L_{\left(x^{-1}, x y\right)}(y)\right]^{-1} \\
& =\left[x y \backslash L_{\left(x^{-1}, x y\right)}(y)\right]^{-1}=\left[x y \backslash L_{\left(x,(x y)^{-1}\right)}(y)\right]^{-1} \\
& =\left[x y \backslash\left(x(x y)^{-1}\right)^{-1}\right]^{-1}=\left[x y \backslash\left(x^{-1}(x y)\right)\right]^{-1} \\
& =\left[x y \backslash L_{x y}\left(x^{-1}\right)\right]^{-1}=\left(x^{-1}\right)^{-1}=x .
\end{aligned}
$$

Portanto,

$$
y \backslash(y x \backslash y)^{-1}=L_{x^{-1}}^{-1} L_{x y}^{-1}(x)=x^{-1} \backslash(x y \backslash x) .
$$

Agora, estamos em condições de demonstrar o teorema 3.3.1

Demonstração (Demonstração do Teorema 3.3.1). Faça $z=x * y$. Então

$$
\begin{aligned}
D_{z}\left(x^{2}\right) & =L_{x^{2}}^{-1}(z)=L_{x^{2}}^{-1}\left(x^{2}\left(x \backslash(x y \backslash x)^{-1}\right)\right)=x \backslash(x y \backslash x)^{-1} \\
& =J\left(x^{-1} \backslash(x y \backslash x)\right)=J\left(y \backslash(y x \backslash y)^{-1}\right) \\
& =J L_{y^{2}}^{-1}\left(y^{2}\left(y \backslash(y x \backslash y)^{-1}\right)\right)=J L_{y^{2}}^{-1}(z)=J D_{z}\left(y^{2}\right) .
\end{aligned}
$$


Onde, nas passagens 3.18 e 3.20 usamos o lema 3.3.2 e na passagem 3.19 usamos o lema 3.3.3. Assim,

$$
x^{2}=D_{z}^{2}\left(x^{2}\right)=D_{z} J D_{z}\left(y^{2}\right)=D_{z^{2}}\left(y^{2}\right)=L_{y^{2}}^{-1}\left(z^{2}\right)
$$

portanto $x^{2} y^{2}=z^{2}$, como queríamos demonstrar.

Em $(Q, *)$, usaremos a notação $S_{x}$ para a translação a esquerda, isto é, $S_{x}:(Q, *) \longrightarrow(Q, *)$ é tal que $S_{x}(y)=x * y$. Notemos que, pelo lema 3.3.2, temos que

$$
S_{x}=L_{x^{2}} L_{x}^{-1} J D_{x} L_{x}
$$

Proposição 3.3.4. Seja $Q$ um A-loop comutativo e seja * como definida em 3.15. Então $(Q, *)$ é um loop comutativo, que associa potências e possui o mesmo elemento neutro de $Q$. Além disso, potências em $(Q, *)$ coincidem com potências em $Q$.

Demonstração. Primeiramente mostremos que a operação * é comutativa. Como $Q$ é um loop comutativo, para quaisquer $x, y \in Q$, temos

$$
x * y=((x y \backslash x)(y x \backslash y))^{-1}=((y x \backslash y)(x y \backslash x))^{-1}=y * x .
$$

Para qualquer que seja $x \in Q$, a identidade 3.21 implica que $S_{x}$ é uma permutação de $Q$, donde segue que $(Q, *)$ é um quase-grupo. Desde que

$$
x * 1=((x \backslash x) \cdot(x \backslash 1))^{-1}=\left(x^{-1}\right)^{-1}=x
$$

para todo $x \in Q$, temos que 1 é o elemento neutro de $(Q, *)$.

Para mostrar que $(Q, *)$ associa potências, façamos

$$
\begin{gathered}
\left(x^{m} * x^{n}\right) * x^{l}=\left(\left(x^{m} x^{n} \backslash x^{m}\right)\left(x^{n} x^{m} \backslash x^{n}\right)\right)^{-1} * x^{l}=\left(\left(x^{m+n} \backslash x^{m}\right)\left(x^{n+m} \backslash x^{n}\right)\right)^{-1} * x^{l}= \\
=\left(x^{-n} x^{-m}\right)^{-1} * x^{l}=x^{m+n} * x^{l}=\left(\left(x^{m+n} x^{l} \backslash x^{m+n}\right)\left(x^{l} x^{m+n} \backslash x^{l}\right)\right)^{-1}= \\
=\left(\left(x^{m+n+l} \backslash x^{m+n}\right)\left(x^{l+m+n} \backslash x^{l}\right)\right)^{-1}=\left(x^{-l} x^{-m-n}\right)^{-1}=x^{m+n+l} .
\end{gathered}
$$


Por outro lado,

$$
\begin{gathered}
x^{m} *\left(x^{n} * x^{l}\right)=x^{m} *\left(\left(x^{n} x^{l} \backslash x^{n}\right)\left(x^{l} x^{n} \backslash x^{l}\right)\right)^{-1}=x^{m} *\left(\left(x^{n+l} \backslash x^{n}\right)\left(x^{l+n} \backslash x^{l}\right)\right)^{-1}= \\
=x^{m} *\left(x^{-l} x^{-n}\right)^{-1}=x^{m} * x^{l+n}=\left(\left(x^{m} x^{l+n} \backslash x^{m}\right)\left(x^{l+n} x^{m} \backslash x^{l+n}\right)\right)^{-1}= \\
=\left(x^{-l-n} x^{-m}\right)^{-1}=\left(x^{-m-n-l}\right)^{-1}=x^{m+n+l}
\end{gathered}
$$

donde temos $\left(x^{m} * x^{n}\right) * x^{l}=x^{m} *\left(x^{n} * x^{l}\right)$, para quaisquer $x \in Q, m, n, l \in \mathbb{Z}$.

Finalmente, mostremos que potências em $(Q, *)$ coincidem com potências em $Q$. De fato,

$$
x^{m}=\left(x^{m-1} * x\right)=\left(\left(x^{m-1} x \backslash x^{m-1}\right)\left(x x^{m-1} \backslash x\right)\right)^{-1}=\left(x^{-1} x^{-m+1}\right)^{-1}=x^{m+1} x,
$$

para todo $x \in Q$ e todo $m \in \mathbb{Z}$.

Lema 3.3.5. Para quaisquer $x, y \in Q$ e $m, n \in \mathbb{Z}, L_{\left(y, x^{m}\right)} S_{x^{n}}=S_{x^{n}} L_{\left(y, x^{m}\right)}$.

Demonstração. Desde que $S_{x^{n}}=L_{x^{2 n}} L_{x^{n}}^{-1} J D_{x^{n}} L_{x^{n}}$, temos que

$$
\begin{aligned}
L_{\left(y, x^{m}\right)} S_{x^{n}} & =L_{\left(y, x^{m}\right)} L_{x^{2 n}} L_{x^{n}}^{-1} J D_{x^{n}} L_{x^{n}} \\
& =L_{x^{2 n}} L_{x^{n}}^{-1} L_{\left(y, x^{m}\right)} J D_{x^{n}} L_{x^{n}} \\
& =L_{x^{2 n}} L_{x^{n}}^{-1} J L_{\left(y, x^{m}\right)} D_{x^{n}} L_{x^{n}} \\
& =L_{x^{2 n}} L_{x^{n}}^{-1} J D_{x^{n}} L_{\left(y, x^{m}\right)} L_{x^{n}} \\
& =L_{x^{2 n}} L_{x^{n}}^{-1} J D_{x^{n}} L_{x^{n}} L_{\left(y, x^{m}\right)}=S_{x^{n}} L_{\left(y, x^{m}\right)} .
\end{aligned}
$$

Para finalizar a seção, notemos um importante resultado.

Teorema 3.3.6. Se $Q$ for uma A-loop comutativo unicamente 2-divisivel, então $(Q, *)$ será isomorfo a $Q$.

Demonstração. Defina $\varphi:(Q, *) \longrightarrow Q$, por $\varphi(x)=x^{2}$. Vamos mostrar que $\varphi$ é um isomorfismo. De fato, como $(x * y)^{2}=x^{2} y^{2}$ para quaisquer $x, y \in Q$, $\varphi$ é um homomorfismo. Desde que $Q$ é unicammente 2-divisível, temos que $\varphi$ é sobrejetiva. Finalmente, se $x^{2}=1$, temos que $x=1$, e assim, $\varphi$ é injetiva. Portanto, $\varphi$ é um isomorfismo. 


\subsection{O Teorema de Decomposição}

Nessa seção mostraremos que um $A$-loop comutativo finito pode ser escrito como produto direto de um subloop de ordem ímpar e um subloop cuja ordem de qualquer elemento é uma potênicia de 2 . Ainda nessa seção, $Q$ denota um $A$-loop comutativo finito. O seguinte teorema é o principal resultado dessa seção.

Teorema 3.4.1. [Decomposição de um A-loop comutativo finito] Se $Q$ for um A-loop comutativo finito, então $Q=K(Q) \times H(Q)$, onde $K(Q)=\{x \in Q:|x|$ é ímpar $\}$ e $H(Q)=\left\{x \in Q: x^{2^{n}}=1\right.$ para algum $\left.n \in \mathbb{Z}\right\}$. Além disso, $K(Q)$ tem ordem impar e $H(Q)$ tem ordem potência de 2.

Agora vamos nos concentrar em alguns resultados, que serão necessários para demonstrarmos o teorema 3.4.1.

Proposição 3.4.2. O conjunto $K_{1}(Q)=\left\{x^{2}: x \in Q\right\}$ é um subloop normal de $Q$.

Demonstração. Claramente $K_{1}(Q)$ é não vazio. Para quaisquer $x, y \in K_{1}(Q)$, temos, pelo teorema 3.3.1, $x^{2} y^{2}=(x * y)^{2}$. Logo $K_{1}(Q)$ é fechado pela multiplicação de $Q$. Além disso, pela proposição 3.3.4, temos que existe $z \in Q$ tal que $x * z=y$ e, novamente pelo teorema 3.3.1, temos $x^{2} z^{2}=y^{2}$. Como $Q$ é comutativo, temos que $K_{1}(Q)$ é um subloop de $Q$.

Resta-nos, agora, mostrar que $K_{1}(Q)$ é normal em $Q$. Como $Q$ é um A-loop, temos que toda aplicaçaõ interna é um automorfismo de $Q$, logo preserva quadrados. Assim $K_{1}(Q)^{\varphi}=K_{1}(Q)$ para toda $\varphi \in \mathcal{I}(L)$. Logo $K_{1}(Q)$ é um subloop normal de $Q$.

Teorema 3.4.3. Seja $Q$ um A-loo comutativo. Para cada $n \in \mathbb{N}$, defina

$$
K_{n}(Q)=\left\{x^{2^{n}}: x \in Q\right\} \quad \text { e } K(Q)=\bigcap_{n \geq 1} K_{n}(Q)
$$

Então: 
1. $K_{n+1}(Q)=\left\{x^{n}: x \in K_{n}(Q)\right\}$, para todo $n \geq 0$;

2. $K_{n+1}(Q)$ é um subloop de $K_{n}(Q)$, para todo $n \geq 0$;

3. $K_{n}(Q) \triangleleft Q$, para todo $n \geq 0$;

4. $K(Q) \triangleleft Q$;

5. Se $Q$ for finito, então $K(Q)=\{x \in Q:|x|$ é ímpar $\}$ e $|K(Q)|$ é ímpar.

\section{Demonstração.}

1. Se $x \in K_{n}(Q)$, temos que $x=y^{2^{n}}$ para algum $y \in Q$. Então $x^{2}=y^{2^{n}} y^{2^{n}}=$ $y^{2^{n+1}} \in K_{n+1}(Q)$. Reciprocamente, se $x \in K_{n+1}(Q)$, então, $x=z^{2^{n+1}}=\left(z^{2^{n}}\right)^{2}$ para algum $z \in Q$.

2. Se $x \in K_{n+1}(Q)$, temos $x=z^{2^{n+1}}=\left(z^{2}\right)^{2^{n}}$, para algum $z \in Q$. Logo, $K_{n+1}(Q) \subset K_{n}(Q)$. Concluimos que $K_{n+1}(Q)$ é um subloop de $K_{n}(Q)$ pelo item 1 e pela proposião 3.4.2.

3. Para provar esse item, vamos proceder por indução sobre n. Pela proposição 3.4.2 temos $K_{1}(Q) \triangleleft Q$. Assuma que $K_{m}(Q) \triangleleft Q$. Queremos mostrar que $K_{m+1}(Q)$ é um subloop normal de $Q$. Como temos que $K_{m+1}(Q)$ é um subloop de $K_{m}(Q)$, segue $K_{m+1}$ é um subloop de $Q$. Para mostrar que $K_{m+1}(Q) \triangleleft Q$, basta observar que qualquer aplicação interna de $Q$ preserva potências, pois $Q$ é um A-loop. Logo, $K_{n}(Q) \triangleleft Q$, para todo $n \geq 0$.

4. Esse item segue do fato de que interseção de uma família de subloops normais de $Q$ é um subloop normal de $Q$.

5. Agora vamos assumir que $Q$ é finito. Temos que

$$
Q \supset K_{1}(Q) \supset K_{2}(Q) \supset \ldots \supset K_{n}(Q) \supset \ldots
$$


Assim existe $n \in \mathbb{N}$ tela que $K(Q)=K_{i}(Q)$, para todo $i \geq n$. Pelo item 1 temos que $K(Q)=\left\{x^{2}: x \in K(Q)\right\}$. Donde temos que $K(Q)$ é um A-loop comutativo, finito, unicamente 2-divisível. Logo, pelo lema 3.2.2, $K(Q)$ tem oredem ímpar. Ainda pelo fato de $\left.K_{(} Q\right)$ ser unicamete 2-divisivel, a aplicação $x \mapsto x^{2}$ é uma bijeção que fixa apenas o elemento 1 , assim nenhum elemento de $\left.K_{(} Q\right)$ pode ter oredem par. Finalmente, seja $x \in Q$ cuja oredem é $2 m+1$. para algum $m \in \mathbb{N}$. Como $x=x^{2 m+2}=\left(x^{m+1}\right)^{2}$, temos que $x \in K_{1}(Q)$. Pelo item 3, temos que $x^{m+1} \in K_{1}(Q)$ e qntão $x \in K_{2}(Q)$ e assim por diante. Logo, $x \in K(Q)$, e assim, $K(Q)=\{x \in Q:|x|$ é ímpar $\}$.

Lema 3.4.4. Para todos $x, y$ em um A-loop comutativo $Q$,

$$
(x \backslash(y \backslash x))^{2} \backslash\left(y^{-1} \backslash(y \backslash x)\right)^{2}=(x \backslash y)^{-2} .
$$

Demonstração. Substitua y por $x \backslash y$ no item 2 do lema 3.1.2, temos

$$
x(x \backslash y)^{2}=(x(x \backslash y))((x . x \backslash y) \backslash x)^{-1}=y(y \backslash x)^{-1} .
$$

Novamente faça $y \backslash x$ no lugar de y e usando o fato que $(y \backslash x) \backslash x=y$, temos

$$
x(x \backslash(y \backslash x))^{2}=(y \backslash x)((y \backslash x) \backslash x)^{-1}=y^{-1}(y \backslash x) .
$$

Aplicando $J$ nos dois lados da equação 3.24 e usando a propriedade (AIP), obtemos

$$
x^{-1}(x \backslash(y \backslash x))^{-2}=y(y \backslash x)^{-1} .
$$

Agora, junte as equações 3.23 e 3.25 afim de obter

$$
(x \backslash y)^{2}(x \backslash(y \backslash x))^{-2}=D_{y(y \backslash x)^{-1}}(x) D_{y(y \backslash x)^{-1}}\left(x^{-1}\right)=\left(y(y \backslash x)^{-1}\right)^{2} .
$$

Finalmente, aplicando $J$ na última equação e usando a (AIP), temos

$$
(x \backslash y)^{-2}(x \backslash(y \backslash x))^{2}=\left(y^{-1}(y \backslash x)\right)^{2},
$$

e então $(x \backslash(y \backslash x))^{2} \backslash\left(y^{-1}(y \backslash x)\right)^{2}=(x \backslash y)^{-2}$. 
Proposição 3.4.5. Seja $x$ em um A-loop comutativo finito $Q$, satisfazendo $x^{2^{n}}=1$. Então, $(x y)^{2^{n}}=y^{2^{n}}$, para todo $y \in Q$.

Demonstração. Vamos proceder por indução sobre $n$. Para o caso $n=0$, temos

$$
(x y)^{2^{0}}=(x y)^{1}=x^{1} y^{1}=x^{2^{0}} y^{2^{0}}=y^{2^{0}} .
$$

Agora assuma que a proposição valha para algum n e tome $x \in Q$ tal que $x^{2^{n+1}}=1$. Desde que $1=x^{2^{n+1}}=\left(x^{2}\right)^{2^{n+1}}$, temos

$$
\left(x^{2} y\right)^{2^{n}}=y^{2^{n}}=\left(x^{2}\left(x^{2} \backslash y\right)^{2^{n}}\right)=\left(x^{2} \backslash y\right)^{2^{n}}
$$

para todo $y \in Q$.

Seja $\varphi$ um automorfismo de $Q$. Aplicando $\varphi$ na equação 3.26 e fazendo $z=y^{\varphi}$, obtemos

$$
\left(\left(x^{\varphi}\right)^{2} z\right)^{2^{n}}=\left(\left(x^{\varphi}\right)^{2} \backslash z\right)^{2^{n}}=z^{2^{n}}, \quad \text { para todo } z \in Q .
$$

Como $Q$ é um A-loop onde vale $(A I P)$, podemos escolher $\varphi=L_{(x, x \backslash y)} J$. Note que

$$
L_{(x, x \backslash y)} J(x)=L_{(x, x \backslash y)}\left(x^{-1}\right)=L_{y^{-1}} L_{x \backslash y} L_{x}\left(x^{-1}\right)=y \backslash(x \backslash y) .
$$

Portanto $\left(z(y \backslash(x \backslash y))^{2}\right)^{2^{n}}=z^{2^{n}}\left((y \backslash(x \backslash y))^{2} \backslash z\right)^{2^{n}}$, para quaisquer $y, z \in Q$. Assim

$$
\begin{aligned}
y^{2^{n+1}} & =\left(y(y \backslash(x \backslash y))^{2}\right)^{2^{n+1}} \\
& =\left(x^{-1}(x \backslash y)\right)^{2^{n+1}} \\
& =\left(\left(x^{-1}(x \backslash y)\right)^{2}\right)^{2^{n}} \\
& =\left[(y \backslash(x \backslash y))^{2} \backslash\left(x^{-1}(x \backslash y)\right)^{2}\right]^{2^{n}} \\
& =(y \backslash x)^{-2^{n+1}} .
\end{aligned}
$$

Então, $\left(y^{-1}\right)^{-2^{n+1}}=y^{2^{n+1}}=L_{\left(y, y^{-1}\right)}\left(y^{2^{n+1}}\right)=\left(L_{\left(y, y^{-1}\right)}(y \backslash x)\right)^{-2^{n+1}}=\left(y^{-1} x\right)^{-2^{n+1}}$. Agora, basta tomar inversos e substituir $y^{-1}$ por $y$, para obtermos o resultado desejado. 
Teorema 3.4.6. Seja $Q$ um A-loop comutativo. Para $n \geq 0$, sejam

$$
\begin{gathered}
H_{n}(Q)=\left\{x \in Q: x^{2^{n}}=1\right\}, \\
H(Q)=\bigcup_{n \geq 0} H_{n}(Q) .
\end{gathered}
$$

Então:

1. $H_{n+1}(Q)=\left\{x \in Q: x^{2} \in H_{n}(Q)\right\}$, para todo $n \geq 0$;

2. $H_{n}(Q) \subset H_{n+1}(Q)$, para todo $n \geq 0$;

3. $H_{n}(Q) \triangleleft Q$, para todo $n \geq 0$;

4. $H(Q) \triangleleft Q$.

\section{Demonstração.}

1. Se $x \in Q$, tal que $x^{2} \in H_{n}(Q)$, então $x^{2^{n+1}}=\left(x^{2}\right)^{2^{n}}=1$, portanto, $x \in$ $H_{n+1}(Q)$. Reciprocamente se $x \in H_{n+1}(Q)$, temos $\left(x^{2}\right)^{2^{n}}=x^{2^{n+1}}=1$, ou seja, $x^{2} \in H_{n}(Q)$.

2. Se $x \in H_{n}(Q)$, então $x^{2^{n+1}}=\left(x^{2^{n}}\right)^{2}=1$, isto é, $x \in H_{n+1}(Q)$.

3. Vamos mostrar que $H_{n}(Q) \leq Q$, para todo $n \geq 0$. Seja $n \in \mathbb{N}$. Claramente, $1 \in H_{n}(Q)$. Pela proposição 3.4.5, temos que, para quaisquer $x, y \in H_{n}(Q)$, $(x y)^{2^{n}}=y^{2^{n}}=1$, ou seja $x y \in H_{n}(Q)$. Também temos que existe $z \in Q$, tal que $x z=y$. Assim $z^{2^{n}}=(x z)^{2^{n}}=y^{2^{n}}=1$, donde temos que $z \in H_{n}(Q)$ e então, $H_{n}(Q)$ é um subloop de $Q$. Para mostrar normalidade, basta observar que, como $Q$ é um A-loop, qualquer aplicação interna de $Q$ é um automorfismo de $Q$ e, portanto, preserva potências. Ou seja, $H_{n}(Q)^{\varphi}=H_{n}(Q)$ para todo $\varphi \in \mathcal{I}(L)$, logo $H_{n}(Q)$ é um subloop normal de $Q$.

4. Sejam $x, y \in H(Q)$. Então existem $n, m \geq 0$ tais que $x \in H_{n}(Q)$ e $y \in H_{m}(Q)$. Tome $k=\max \{m, n\}$. Claramente $x y \in H(Q)$. Existe $z \in Q$, tal que, $x z=y$. 
Então, pela proposição 3.4.5 temos $z^{2^{k}}=(x z)^{2^{k}}=y^{2^{k}}=1$, ou seja, $z \in$ $H_{k}(Q) \subset H(Q)$, logo $H(Q)$ é um subloop de $Q$. Pelo mesmo argumento do item 3, temos que $H(Q)^{\varphi}=H(Q)$ para todo $\varphi \in \mathcal{I}(L)$, isto é, $H(Q)$ é um subloop normal de $Q$.

Demonstração (Demostração do Teorema 3.4.1). Pelos teoremas 3.4.3 e 3.4.6, temos que $K(Q), H(Q) \triangleleft Q$ e $K(Q) \cap H(Q)=\{1\}$. Resta-nos agora mostrar que $Q=K(Q) H(Q)$. Seja $x \in Q$ e faça $|x|=n=2^{k} . m$ onde $\left(2^{k}, m\right)=1$. Então existem $a, b \in \mathbb{Z}$ tais que $1=2^{k} a+m b$. Assim,

$$
x=x^{1}=x^{2^{k} a+m b}=x^{2^{k} a} \cdot x^{m} .
$$

Logo $x_{1}=x^{2^{k} a} \in K(Q)$ e $x_{2}=x^{m b} \in H(Q)$, como queríamos demonstrar.

\subsection{A-Loops Comutativos de Expoente 2}

A definição de expoente para um loop finito é a mesma usada para grupos, isto é, o expoente de um loop finito é o mínimo múltiplo comum da ordem de seus elementos. A proposição abaixo nos motiva a estudar os $A$-loops comutativos de expoente 2 .

Proposição 3.5.1. Um A-loop comutativo, finito e simples ou é um grupo cíclico de ordem p, para algum número primo ímpar p, ou tem expoente 2.

Demonstração. Seja $Q$ um A-loop comutativo finito e simples. Pelo Teorema 3.4.5, $Q=K \times H$, onde $K$ tem ordem impar e $H=\left\{x \in Q: x^{2^{n}}=1\right.$ para algum $\left.n \geq 0\right\}$. Como, $Q$ é simples, temos que $Q=K$ ou $Q=H$

Se $Q=K$, pelo Teorema da Ordem Ímpar (3.2.17), temos que Q é solúvel. Assim, $Q$ simples, solúvel e comutativo implica que $Q$ é um grupo cíclico de oredem prima $p$. Agora assuma $Q=H$, então todo elemento de $Q$ tem ordem potência de 2. Da proposição 3.4.2, que $K_{1}(Q) \triangleleft Q$. Como $Q$ é simples, temos $K_{1}(Q)=Q$ ou $K_{1}(Q)=$ $\{1\}$. Se valesse o primeiro caso, $Q$ seria um A-loop comutativo, finito e unicamente 2-divisível, assim pela proposição 3.2.2, Q teria ordem impar, o que contradiz o fato 
de $|H|$ ser u a potência de 2. Logo, devemos ter $K_{1}(Q)=\{1\}$ e assim, $x^{2}=1$ para todo $x \in Q$, isto é, $Q$ tem expoente 2.

Teorema 3.5.2. Seja $Q$ um A-loop comutativo de expoente 2. Então, $(Q, *)$ é um 2-grupo abeliano elementar.

Antes de demonstrarmos a proposição 3.5.2 façamos alguns resultados que serão necessários. Lembremos que * é definido por 3.15 e 3.16, ou seja,

$$
x * y=((x y \backslash x)(y x \backslash y))^{-1}=L_{(y, x)}(y) L_{(x, y)}(x) .
$$

Como consequência imediata do teorema 3.5.2 temos que se $Q$ for um $A$-loop comutativo finito e de expoente 2 , então $|Q|$ será uma potência de 2 .

Nessa seção $Q$ será um $A$-loop comutativo de expoente 2. Vimos que

$$
x * y=x^{2}\left(x \backslash(x y \backslash x)^{-1}\right) \quad \text { e } \quad S_{x}=L_{x^{2}} L_{x}^{-1} D_{x} L_{x}
$$

mas, desde que $Q$ tem expoente 2, obtemos

$$
x * y=x \backslash(x y \backslash x) \quad \text { e } \quad S_{x}=L_{x}^{-1} D_{x} L_{x}
$$

Lema 3.5.3. Para todos $x, y \in Q, x *(x * y)=y$, isto é $S_{x}^{2}=i d_{Q}$.

Demonstração. De fato, como $S_{x}=L_{x}^{-1} D_{x} L_{x}$ e $D_{x}^{2}=i d_{Q}$, temos que

$$
S_{x}^{2}=\left(L_{x}^{-1} D_{x} L_{x}\right)\left(L_{x}^{-1} D_{x} L_{x}\right)=L_{x}^{-1} D_{x}^{2} L_{x}=L_{x}^{-1} L_{x}=i d_{Q} .
$$

Lema 3.5.4. Para todo $x \in Q, S_{x}=L_{x}^{-1} D_{x} L_{x}=L_{x} D_{x} L_{x}^{-1}$.

Demonstração. Como $Q$ tem expoente 2, vale que $D_{x}=D_{L_{x}^{2}(x)}$ e $L_{x}^{2}=L_{(x, x)} \in$ $\mathcal{I}(L)$, ou seja, $D_{x} L_{x}^{2}=D_{L_{x}^{2}(x)} L_{x}^{2}$. Temos que

$$
D_{L_{x}^{2}(x)}\left(L_{x}^{2}(y)\right)=\left[L_{x}^{2}(y) \backslash\left(L_{x}^{2}(x)\right)\right]=L_{x}^{2}(y \backslash x)=L_{x}^{2}\left(D_{x}(y)\right)
$$

para todo $y \in Q$, isto é $D_{x} L_{x}^{2}=L_{x}^{2} D_{x}$ ou, equivalentemente, $L_{x}^{-1} D_{x} L_{x}=L_{x} D_{x} L_{x}^{-1}$. 
Lema 3.5.5. Para quaisquer $x, y, z \in Q$

$$
S_{z y} L_{(z \backslash(x(z y)), z)}(y)=L_{x} D_{y} L_{x}^{-1} L_{y}(z) .
$$

Demonstração. Primeiramente, façamos

$$
\begin{aligned}
L_{z x} L_{z x \backslash z y}^{-1} S_{z y} L_{(x, z)}(y) & =\left(L_{z x} L_{z x \backslash z y}^{-1} L_{z y}\right) L_{z y}^{-1} S_{z y} L_{(x, z)}(y) \\
& =\left(L_{(x z, y z)} L_{y z}^{-1}\right) S_{z y} L_{(x, z)}(y) \\
& =L_{z y}^{-1}\left(L_{(z x, z y)} S_{z y}\right) L_{(x, z)}(y) \\
& =L_{z y}^{-1} S_{z y}\left(L_{(z x, z y)} L_{(x, z)}\right)(y) \\
& =L_{z y}^{-1} S_{z y} L_{(z x)(z y)}^{-1} L_{z y} L_{z} L_{x}(y) \\
& =D_{z y} L_{z y}^{-1} L_{(z x)(z y)}^{-1} L_{z y} L_{z} L_{y}(x),
\end{aligned}
$$

onde usamos os lemas 3.3.5 e 3.5.4 para justificar as passagens 3.28 e 3.29, respectivamente.

Como $Q$ tem expoente 2 , temos que $L_{z y} L_{z} L_{y}(1)=1$, ou seja, $L_{z y} L_{z} L_{y} \in \mathcal{I}(L)$, o que implica

$$
L_{z y} L_{z} L_{y}(y \backslash x)=L_{z y} L_{z} L_{y}(y) \backslash L_{z y} L_{z} L_{y}(x) .
$$

Por outro lado, $L_{z y} L_{z} L_{y}(y \backslash x)=L_{z y} L_{z} L_{y} L_{y}^{-1}(x)=L_{z y} L_{z}(x)=(z x)(z y)$. Então

$$
L_{z y} L_{z} L_{y}(x)=[(z x)(z y)] L_{z y} L_{z} L_{y}(y)=L_{(z x)(z y)} L_{z y} L_{z} L_{y}(y) .
$$

Desde que $y=L_{y \backslash x}^{-1}(x)$, a última equação ode ser reescrita como

$$
L_{z y} L_{z} L_{y} L_{y \backslash x}^{-1}(x)=L_{(z x)(z y)}^{-1} L_{z y} L_{z} L_{y}(x) .
$$

Assim obtemos

$$
\begin{aligned}
L_{z x} L_{z x \backslash z y}^{-1} S_{z y} L_{(x, z)}(y) & =D_{z y} L_{z y}^{-1}\left(L_{(z x)(z y)}^{-1} L_{z y} L_{z} L_{y}(x)\right) \\
& =D_{z y} L_{z} L_{y} L_{y \backslash x}^{-1}(x) \\
& =D_{z y} L_{z} L_{y}(y)=D_{z y}(z)=y .
\end{aligned}
$$


Portanto, $S_{z y} L_{(x, z)}(y)=L_{z x \backslash z y} L_{z x}^{-1}(y)=L_{z x \backslash z y} L_{(z y \backslash z x) \backslash z y}^{-1}(y)$. Agora, substitua $x$ por $L_{z}^{-1} L_{z y}(x)=z \backslash(x(z y))$ para obter

$$
\begin{aligned}
S_{z y} L_{(z \backslash(x(z y)), z)}(y) & =L_{x} L_{x \backslash(z y)}^{-1}(y) \\
& =L_{x}((x \backslash z y) \backslash y) \\
& =L_{x} D_{y}(x \backslash z y) \\
& =L_{x} D_{y} L_{x}^{-1}(z y)=L_{x} D_{y} L_{x}^{-1} L_{y}(z),
\end{aligned}
$$

como era desejado.

Lema 3.5.6. Para todos $u, v, w \in Q$,

$$
L_{(v \backslash(w . u v), v)}(u)=L_{w} D_{v} L_{w}^{-1} L_{v}(u) .
$$

Demonstração. De fato, para quaisquer $u, v, w \in Q$, temos

$$
\begin{aligned}
L_{(v \backslash(w . u v), v)}(u) & =L_{w \cdot u v}^{-1} L_{v}\left[L_{v \backslash(w . u v)}(u)\right]=L_{w \cdot u v}^{-1} L_{v}\left[L_{u} L_{v}^{-1} L_{u v}\right](w) \\
& =L_{w \cdot u v}^{-1} L_{v} L_{(u, u v)}(w)=L_{w \cdot u v}^{-1} L_{v} L_{(v \backslash(u v), u v)}(w) \\
& =L_{w \cdot u v}^{-1}\left[L_{v} L_{(u v \backslash v, u v)}\right](w)=L_{w \cdot u v}^{-1} L_{u v}\left(L_{u v \backslash v}(w)\right) \\
& =L_{w \cdot u v}^{-1} L_{u v} L_{w}(u v \backslash v)=L_{(w, u v)} L_{u v}^{-1}(v) \\
& =L_{w} L_{w \backslash u v}^{-1}(v)=L_{w} D_{v} L_{w}^{-1} L_{v}(u) .
\end{aligned}
$$

Lema 3.5.7. Para todos $u, v, w \in Q$,

$$
L_{(v w, u)} L_{v} L_{v \backslash w}^{-1}(u)=w u
$$

Demonstração. Novamente, para quaisquer $u, v, w \in Q$, temos

$$
\begin{aligned}
L_{(v w, u)}\left[L_{v} L_{v \backslash w}^{-1}\right](u) & =L_{(v w, u)} L_{w}^{-1} L_{(v, w)}(u)=\left[L_{(v w, u)} L_{(v, w)}\right] L_{w}^{-1}(u) \\
& =L_{v w \cdot u}^{-1} L_{u} L_{w} L_{v} L_{w}^{-1}(u)=L_{v w \cdot u}^{-1} L_{u} L_{w} L_{w \backslash u}(v) \\
& =L_{v w \cdot u}^{-1} L_{u w}\left[L_{(w, u)} L_{w \backslash u}\right](v)=L_{v w \cdot u}^{-1} L_{w u} L_{u} L_{w}(v) \\
& =L_{w v \cdot u}^{-1}((w v \cdot u) w u)=w u
\end{aligned}
$$


Lema 3.5.8. Para todos $u, v, w \in Q$,

$$
S_{u v} L_{(w, u)}(v)=L_{v} L_{u}^{-1} L_{(w, u)}(v) .
$$

Demonstração. Vamos começar com

$$
S_{u v} L_{(u \backslash(w . u v), u)}(v)=L_{w} D_{v} L_{w}^{-1} L_{v}(u)=L_{(v \backslash(w . u v), v)} .
$$

Substituindo w por $L_{u} L_{u v}^{-1}(w)$, obtemos

$$
\begin{aligned}
S_{u v} L_{(w, u)}(v) & =L_{(v \backslash w u, v)}(u)=L_{(w v \backslash v, v)}(u) \\
& =L_{v} L_{(w u) \backslash v} L_{(w u \backslash v) \backslash v}^{-1}(u)=L_{v} L_{w u \backslash v} L_{w u}^{-1}(u) \\
& =L_{v} L_{w u \backslash u} L_{w u}^{-1}(v)=L_{v} L_{u \backslash w u} L_{(u \backslash u w) \backslash u}^{-1}(v) \\
& =L_{v} L_{w} L_{w u}^{-1}(v)=L_{v} L_{u}^{-1} L_{(w, u)}(v) .
\end{aligned}
$$

Lema 3.5.9. Para todo $x, y \in Q$,

$$
L_{x} D_{y} L_{x}^{-1}=D_{x y} L_{y} D_{x} L_{x}^{-1} .
$$

Demonstração. Seja $z \in Q$. Temos

$$
\begin{aligned}
L_{x} D_{y} L_{x}^{-1} L_{y}(z) & =S_{z y} L_{(z \backslash(x . y z), z)}(y) \\
& =S_{z y} L_{x} D_{z} L_{x}^{-1} L_{z}(y) \\
& =S_{z y} L_{x} D_{z}\left[L_{x}^{-1} L_{z} L_{z \backslash x}\right] L_{z \backslash x}^{-1}(y) \\
& =S_{z y} L_{x} D_{z} L_{(z \backslash x, z)} L_{z \backslash x}^{-1} \\
& =S_{z y} L_{x} L_{(z \backslash x, z)} D_{z} L_{z \backslash x}^{-1}(y) \\
& =S_{z y} L_{z} L_{z \backslash x} D_{z} L_{z \backslash x}^{-1},
\end{aligned}
$$

onde nas passagens 3.34 e 3.35 foram usados as identidades 3.27 e 3.30, respectivamente.

Agora faça $u=L_{z \backslash x} D_{z} L_{z \backslash x}^{1}(y)=L_{z \backslash x} L_{(z \backslash x) \backslash y}(z)$ e observe que, pela equação 3.31,

$$
L_{((z \backslash x) y, z)}(u)=L_{((z \backslash x) y, z)} L_{z \backslash x} L_{(z \backslash x) \backslash y}(z)=y z .
$$


Usando que * é comutativa obtemos

$$
\begin{aligned}
L_{x} D_{y} L_{x}^{-1} L_{y}(z) & =S_{z y} L_{z} L_{z \backslash x} L_{(z x) \backslash y}^{-1}(z) \\
& =S_{z y} L_{z}(u)=S_{z y}(z u) \\
& =S_{z u}(z y)=S_{z u} L_{((z \backslash x) y, z)}(u) \\
& =L_{u} L_{z}^{-1} L_{((z \backslash x) y, z)}(u)=L_{u} L_{z}^{-1}(y z) \\
& =L_{u}(y)=L_{y}(z) \\
& =L_{y} L_{z \backslash x} L_{(z \backslash x) \backslash y}^{-1}(z) \\
& =L_{(z \backslash x, y)}(z)=L_{(z \backslash x) y}^{-1} L_{y} L_{z \backslash x}(z) \\
& =L_{(z \backslash x) y}^{-1}(y x)=D_{x y} L_{y} D_{x}(z),
\end{aligned}
$$

assim, $L_{x} D_{y} L_{x}^{-1}=D_{x y} L_{y} D_{x} L_{y}^{-1}$, como queríamos demonstrar.

Lema 3.5.10. Para todos $x, y \in Q$, temos $L_{x} D_{y} L_{x}^{-1}=L_{x y} S_{(x y) \backslash x} L_{x y}^{-1}$.

Demonstração. De fato, temos que

$$
\begin{gathered}
L_{x} D_{y} L_{x}^{-1}=L_{x} L_{y} S_{y} L_{y}^{-1} L_{x}^{-1}=L_{x y} L_{(y, x)} S_{y} L_{y}^{-1} L_{x}^{-1}= \\
=L_{x y} S_{L_{(y, x)(y)}} L_{(y, x)} L_{y}^{-1} L_{x}-1=L_{x y} S_{(x y) \backslash x} L_{x y}^{-1}
\end{gathered}
$$

onde, na última passagem, foi usado o lema 3.1.2.

Agora podemos demonstrar o teorema 3.5.2, que diz que se $Q$ for um $A$-loop comutativo de expoente 2 , então $(Q, *)$ será um 2-grupo abeliano elementar.

Demonstração (Demonstração do Teorema 3.5.2). Já temos que $x * x=x^{2}=1$, para todo $x \in Q$.Resta-nos provar que *é associativa.

Pela equação 3.33 temos $L_{x} D_{y} L_{x}^{-1}=D_{x y} L_{y} D_{x} L_{x}^{-1}$. Aplicando o lema 3.5.10 nos dois lados dessa última equação, obtemos $L_{x y} S_{(x y) \backslash x} L_{x y}^{-1}=D_{x y} L_{y x} S_{(y x) \backslash y} L_{y x}^{-1}$, ou seja,

$$
S_{(y x) \backslash x}=S_{(y x) \backslash y} L_{y x} D_{x y} L_{x y}^{-1}=S_{(y x) \backslash y} S_{x y} .
$$


Agora, substituindo $x$ por $y \backslash x$, temos $S_{x \backslash(y \backslash x)}=S_{x} S_{x \backslash y}$. Novamente, troque y por $x y$ e, assim, temos $S_{x \backslash(x y \backslash x)}=S_{x} S_{y}$. Em outras palavras temos

$$
S_{x * y}=S_{y} S_{x},
$$

o que é equivalente $a$ * ser associativa. Logo $(Q, *)$ é um 2-grupo elementar. 


\section{$3.6 \quad p$-Loops}

Seja $p$ um número primo. Um p-grupo é um grupo cuja ordem de qualquer um dos seus elementos é uma potência de $p$. Para grupos finitos essa definição é equivalente a dizer que a ordem do grupo é uma potência de $p$. Felizmente esse resultado também vale para $A$-loops comutativos finitos.

Teorema 3.6.1. Seja $Q$ um A-loop comutativo finito e seja p um número primo. Então $|Q|$ é uma potência de $p$ se e somente se todo elemento de $Q$ tem ordem potência de $p$.

Demonstração. Primeiramente, vamos supor p ímpar. Se $|Q|$ for uma potência de $p$, pela proposição 3.2.9 todo elemento de $Q$ deve ter ordem potência de p. Reciprocamente de $|Q|$ for divisivel por um outro primo ímpar $q$, então $Q$ deve possuir um elemento de ordem q, então, $|Q|$ tem ordem potência de p. Agora analisemos o caso em que $p=2$. Se $|Q|$ for uma potência de 2, pelo teorema 3.4.1 temos que $K(Q)=\{1\}$, e assim todo elemento de $Q$ tem ordem potência de 2. Agora suponha que todo elemento de $Q$ tenha ordem potência de 2. Assuma que $Q$ seja o menor A-loop finito cujo expoente é uma potência de 2 tal que $|Q|$ não seja potência de 2. Pelo teorema 3.4.6, $H_{1}(Q)=\left\{x \in Q: x^{2}=1\right\} \triangleleft Q$. Como consequência do teorema 3.5.2, temos que $\left|H_{1}(Q)\right|=2^{n}$ para algum n. Assim $H_{1}(Q) \neq Q$ e, pela minimalidade de $|Q|$, temos que $\left|Q / H_{1}(Q)\right|$ é uma potência de 2. Logo $|Q|=\left|H_{1}(Q)\right| \cdot\left|Q / H_{1}(Q)\right|$ deve ser uma potência de 2 , o que é uma contradição da escolha de $Q$.

Apresentemos aqui o teorema mais importante desse capítulo, que garante que propriedades importantes de grupos valem para $A$-loops comutativos finitos.

Teorema 3.6.2. [Lagrange, Cauchy] Seja $Q$ um A-loop comutativo finito. Então:

(i) se $x \in A \leq Q$, então $|x|$ e $|A|$ dividem $|Q|$;

(ii) se um primo $p$ divide $|Q|$, então $Q$ possui um elemento de ordem $p$. 
Demonstração. Seja $Q$ um A-loop comutativo finito. Então $Q=K(Q) \times H(Q)$ onde $K(Q)$ é o subloop formado pelos elementos de $Q$ que têm ordem ímpar $|K(Q)|$ é ímpar) e $H(Q)$ é o subloop formado pelos elementos de $Q$ que têm ordem potência de $2(|H(Q)|$ é par $)$. Se $|Q|=2^{k} . m$ onde $m$ é ímpar, então $|K(Q)|=m$ e $|H(Q)|=2^{k}$.

(i) Seja A um subloop de $Q$. Então, A é um A-loop comutativo finito. Então pelo teorema 3.4.1 temos que $A=K(A) \times H(A)$, onde $K(A)$ e $H(A)$ são como descrito acima. Se $y \in A$, então $y=y_{1} y_{2}$, onde $y_{1} \in K(A)$ e $y_{2} \in H(A)$. Como $K(A) \leq K(Q)$, pela proposição 3.2.9, temos que, $\left|y_{1}\right|$ divide $|K(A)|$ e $|K(A)|$ divide $|K(Q)|$. Do mesmo modo $H(A) \leq H(Q)$, assim $\left|y_{2}\right|$ divide $|H(A)|$ que, por sua vez, divide $|H(Q)|$. Logo $|y|$ divide $|A|$ que divide $|Q|$.

(ii) Seja p um número primo que divide $|Q|$. Se p é ímpar, temos que $K(Q) \neq\{1\}$ e, pela proposição 3.2.10, temos que $Q$ possui um elemento de ordem $p$. Se $p=2$, então $H(Q) \neq\{1\}$ e assim existe $x \in Q$, com $|x|=2^{n}$, para algum $n$. Então $x^{2^{n}}=\left(x^{2^{n-1}}\right)^{2}=1$, então $x^{2^{n-1}}$ é o elemento procurado.

Observação. Se, para um A-loop comutativo finito de ordem ímpar, valer que existem subloops de Hall e Sylow, pelo Teorema da Decomposição, mostra-se que para todo A-loop comutativo finito existem subloops de Hall e Sylow. 


\section{Capítulo 4}

\section{A-Loops Comutativos Finitos: Construções}

\subsection{Loops comutativos cujo núcleo intermediário tem índice 2}

Para um conjunto $X$, denotaremos $\bar{X}=\{\bar{x}: x \in X\}$ uma cópia disjunta do conjunto $X$. Sejam $G$ um grupo abeliano, com elemento neutro 1, e $f$ um permutação de $G$. Considere no cojunto $G(f)=G \cup \bar{G}$ a seguinte operação binária:

$$
x * y=x y, \quad x * \bar{y}=\overline{x y}, \quad \bar{x} * y=\overline{x y} \quad \text { e } \quad \bar{x} * \bar{y}=f(x y),
$$

para quaisquer $x, y \in G$. Afirmamos que $(G(f), *)=G(f)$ é um loop com elemento neutro 1. De fato, sejam $a, b \in G$ temos que:

- $x=a^{-1} b$ é a única solução de $a * x=b$;

- $x=\overline{a^{-1} f^{-1}(b)}$ é a única solução de $\bar{a} * x=b$;

- $x=a^{-1} b$ é a única solução de $\bar{a} * x=\bar{b}$;

- $x=\overline{a^{-1} b}$ é a única solução de $a * x=\bar{b}$; 
Temos que $G(f)$ é comutativo (lema 4.1.3) e assim, temos que $G(f)$ é um quase-grupo. Finalmente, vale que

$$
a * 1=a \quad \text { e } \quad \bar{a} * 1=\bar{a} .
$$

Portanto $G(f)$ é um loop com elemento neutro 1

Observação 4.1.1. $Q=G(f)$ será um grupo se e somente se $\overline{1} \in \mathcal{N}_{\mu}(Q)$.

Se $Q$ for um grupo teremos $Q=\mathcal{N}_{\mu}(Q)$ e assim, é claro que, $\overline{1} \in \mathcal{N}_{\mu}(Q)$. Reciprocamente, vamos supor que $\overline{1} \in \mathcal{N}_{\mu}(Q)$. Sejam $x, y, z \in G$. Temos que $G \leq \mathcal{N}_{\mu}(Q)$ (lema 4.1.3), então vale que $\bar{x} *(y * \bar{z})=(\bar{x} * y) * \bar{z}, \bar{x} *(y * z)=(\bar{x} * y) * z$, $x *(y * \bar{z})=(x * y) * \bar{z}$ e $x *(y * z)=(x * y) * z$. Agora temos que

$$
\begin{aligned}
x *(\bar{y} * z) & =x *((y * \overline{1}) * z) \\
& =x *(y *(\overline{1} * z)) \\
& =(x * y) *(\overline{1} * z) \\
& =((x * y) * \overline{1}) * z) \\
& =(x *(y * \overline{1})) * z \\
& =x *(\bar{y} * z) .
\end{aligned}
$$

Da mesma forma podemos mostrar que $\bar{x} *(\bar{y} * z)=(\bar{x} * \bar{y}) * z, x *(\bar{y} * \bar{z})=(x * \bar{y}) * \bar{z}$ e $\bar{x} *(\bar{y} * \bar{z})=(\bar{x} * \bar{y}) * \bar{z}$. Logo $Q$ é um grupo.

Observação 4.1.2. Se $Q$ não for um grupo, então $G=\mathcal{N}_{\mu}(Q)$.

Pelo lema 4.1.3 já temos que $G \subset \mathcal{N}_{\mu}(Q)$. Suponha $x \in G$ tal que $\bar{x} \in \mathcal{N}_{\mu}(Q)$. Então para quaisquer $a, b \in Q$ temos

$$
\begin{aligned}
a *(\overline{1} * b) & =a *\left(\left(\bar{x} * x^{-1}\right) * b\right)=a *\left(\bar{x} *\left(x^{-1} * b\right)\right)=(a * \bar{x}) *\left(x^{-1} * b\right)= \\
& =\left((a * \bar{x}) * x^{-1}\right) * b=\left(a *\left(\bar{x} * x^{-1}\right)\right) * b=(a * \overline{1}) * b,
\end{aligned}
$$

assim, $\overline{1} \in \mathcal{N}_{\mu}(Q)$ o que contradiz o fato de $Q$ não ser um grupo. Logo $\mathcal{N}_{\mu}(Q) \subset G$ e, então, $G=\mathcal{N}_{\mu}(Q)$. 
Lema 4.1.3. Sejam $G$ um grupo abeliano e $f$ uma permutação de $G$. Conidere o loop $Q=G(f)$ como definido em 4.1. Então.

(i) Q é comutativo;

(ii) $x \backslash y=x^{-1} y, x \backslash \bar{y}=\overline{x^{-1} y}, \bar{x} \backslash y=\overline{x^{-1} f^{-1}(y)}$ e $\bar{x} \backslash \bar{y}=x^{-1} y$ para todos $x, y \in G$;

(iii) $G \leq \mathcal{N}_{\mu}(Q)$;

(iv) Q será um grupo se e somente se f for uma translação de $G$;

(v) Se $Q$ não for um grupo (isto é, $G=\mathcal{N}_{\mu}(Q)$ ) então $\mathcal{N}_{\lambda}(Q)=\mathcal{N}_{\rho}(Q)=\mathcal{Z}(Q)=$ $\{x \in G: f(x)=x f(1)\}$.

Demonstração. Da definição 4.1 e do fato de $G$ ser um grupo abeliano temos claramente que $Q$ é comutativo. A demosntração do ítem (ii) segue do argumento usado para mostrar que $Q$ é um loop. Para mostrar o item (iii), tome g, $x, y \in G$. Então

$$
\begin{gathered}
x *(g * y)=(x * g) * y, \\
\bar{x} *(g * y)=\bar{x} *(g y)=\overline{x g y}=\overline{x g} * y=(\bar{x} * g) * y, \\
x *(g * \bar{y})=x * \overline{g y}=\overline{x g y}=(x g) * \bar{y}=(x * g) * \bar{y} \quad e \\
\bar{x} *(g * \bar{y})=\bar{x} * \overline{g y}=f(x g y)=\overline{x g} * \bar{y}=(\bar{x} * g) * \bar{y} .
\end{gathered}
$$

Assim, $G \leq \mathcal{N}_{\mu}(Q)$. Na demonstração do item (iv) vamos usar a observação 4.1.1. Desde que $(x * \overline{1}) * \bar{y}=x *(\overline{1} * \bar{y})$ se e somente se $f(x y)=x f(y),(\bar{x} * \overline{1}) * y=\bar{x} *(\overline{1} * y)$ se e somente se $f(x y)=f(x) y$ e $(\bar{x} * \overline{1}) * \bar{y}=\bar{x} *(\overline{1} * \bar{y})$ se e somente se $f(x) y=x f(y)$, temos que $\overline{1} \in \mathcal{N}_{\mu}(Q)$ se e somente se $f(x y)=x f(y)=f(x) y$ para todo $x, y \in G$. Fazendo $y=1$ nessa última igualdade temos que $f(x)=x f(1)$, para todo $x \in G$ o que nos diz que $f$ é uma translação de $G$. Reciprocamente, se $f(x)=x f(1)$, para todo $x \in G$, $f(x y)=x y f(1)=x f(y)$ e $f(x y)=x y f(1)=y f(x)$, donde temos que $1 \in \mathcal{N}_{\mu}(Q)$. Finalmente vamos mostrar que $\mathcal{N}_{\lambda}(Q)=\mathcal{N}_{\rho}(Q)=\mathcal{Z}(Q)=\{x \in G: f(x)=x f(1)\}$ sempre que $Q$ não for um grupo. Para quaisquer $x, y, z \in G$ temos $(x * y) * z=x *(y * z)$ 
$(x * \bar{y}) * z=\overline{x y z}=x *(\bar{y} * z) e(x * y) * \bar{z}=\overline{x y z}=x *(y * \bar{z})$. Então $x \in \mathcal{N}_{\lambda}(Q)$ se e somente se $(x * \bar{y}) * \bar{z}=x *(\bar{y} * \bar{z})$ o que é equivalente a $x f(y z)=f(x y z)$ para todo $y, z \in G$. Fazendo $y=z=1$ temos $f(x)=x f(1)$. Reciprocamente $f(x)=x f(1)$ para todo $x \in G$, teremos que $f(x y z)=x(y z f(1))=x f(y z)$ para todos $y, z \in G$. Como $Q$ é comutativo temos que $\mathcal{N}_{\lambda}(Q)=\mathcal{N}_{\rho}(Q)$ e, por hipótese $\mathcal{N}_{\mu}(Q)=G$, donde segue o resultado.

Lema 4.1.4. Seja $Q$ um loop comutativo com um subloop $G$ satisfazendo $G \leq \mathcal{N}_{\mu}(Q)$ $e[Q: G]=2$. Então $G$ é um grupo abeliano e existe $f$ permutação de $G$ tal que $Q$ é isomorfo a $G(f)$, onde $G(f)$ é definido pela operação 4.1 .

Demonstração. Como $G \subset \mathcal{N}_{\mu}(Q)$, é claro que $G$ é um grupo abeliano. Por hipótese, temos que $[Q: G]=2$ e então $Q \backslash G \neq \emptyset$. Escolha um elemento $\overline{1} \in Q \backslash G$. Para cada elemento $x \in G$ denote por $\bar{x}=x \overline{1}=\overline{1} x$. Se existisse $y \in G \cap \bar{G}$, onde $\bar{G}=\{\bar{g}: g \in G\}$, teríamos $y=\overline{1} z$ para algum $z \in G$ e, assim, como $G$ é um subloop de $Q$, teríamos $\overline{1} \in G$. Então temos que $G \cap \bar{G}=\emptyset$. Se $x_{1}, x_{2} \in G$ são tais que $\overline{x_{1}}=\overline{x_{2}}$, então de $x_{1} \overline{1}=x_{2} \overline{1}$ temos $x_{1}=x_{2}$. Logo a aplicação $:: G \longrightarrow Q \backslash G$ é injetiva e desde que $|G|=|Q \backslash G|$ temos que $\bar{G}=Q \backslash G$ e assim $Q=G \cup \bar{G}$ e essa reunião é disjunta. Se $x \in G$ é tal que $\overline{1}(x \overline{1})=\bar{z} \in \bar{G}$, então $\bar{x}=z \in G \cap \bar{G}$. Logo a aplicação $f: G \longrightarrow G$ dada por $f(x)=\overline{1}(x \overline{1})$ está bem definida. Além disso $f$ é injetiva, pois $\overline{1}(x \overline{1})=\overline{1}(y \overline{1})$ implica $x \overline{1}=y \overline{1}$ e assim $x=y$. Para cada $g \in G$ existe $\alpha=\bar{z} \in \bar{G}$ tal que $\overline{1} \alpha=x$ (pois $Q$ é um loop), então $f(z)=x$ e então $f$ é uma bijeção de $G$. Agora só nos resta mostrar que $Q$ é isomorfo a $G(f)$. Para $x, y \in G$, vale que $x \bar{y}=x(y \overline{1})=\overline{x y}$ e $\bar{x} y=(\overline{1} x) y=\overline{x y}$, e finalmete

$$
\overline{x y}=(\overline{1} x)(y \overline{1})=\overline{1}((x y) \overline{1})=f(x y) .
$$

Assim, a estrutura de loop $Q$ coincide com a estrutura de $G(f)$.

Corolário 4.1.5. Seja $Q$ um loop comutativo. Então se $\left[Q: \mathcal{N}_{\mu}(Q)\right]=2$ existe $G$, grupo abeliano, e $f$, permutação de $G$, tal que $G(f)$ é isomorfo a $Q$. Por outro lado, se existe $G$ grupo abeliano e $f$ permutação de $G$ tal que $Q$ é isomorfo a $G(f)$ então, $\left[Q: \mathcal{N}_{\mu}(Q)\right] \leq 2$. 
Agora trataremos das possíveis relações entre os loops $G\left(f_{1}\right)=G_{1}$ e $G\left(f_{2}\right)=$ $G_{2}$, para distintas $f_{1}, f_{2}$ permutações de $G$. De fato, exibiremos um critério para determinar quando $G_{1} \simeq G_{2}$.

Proposição 4.1.6. Sejam $G$ um grupo e $f_{1}, f_{2}$ permutações de $G$ tais que $G_{1}$ e $G_{2}$ não sejam grupos. Então $G_{1} \simeq G_{2}$ se e somente se existir $\psi \in \operatorname{Aut}(G)$ tal que

$$
f_{2}^{-1} \psi f_{1}(x)=f_{2}^{-1} \psi f_{1}(1) . \psi(x) \quad \text { para todo } \quad x \in G
$$

e ainda se $f_{2}^{-1} \psi f_{1}(1)$ for um quadrado em $G$.

Demonstração. Denotaremos por * a multiplicação em $G_{1}$ e por o a multiplicação de $G_{2}$. Vamos assumir que existe $\phi: G_{1} \longrightarrow G_{2}$ um isomorfismo. Como $G_{1}$ e $G_{2}$ não são grupos, temos que $\mathcal{N}_{\mu}\left(G_{1}\right)=G=\mathcal{N}_{\mu}\left(G_{2}\right)$ e, assim, $\psi=\left.\phi\right|_{G}$ é uma permutação de G. Além disso

$$
\psi(x y)=\phi(x y)=\phi(x * y)=\phi(x) \circ \phi(y)=\phi(x) \phi(y)=\psi(x) \psi(y)
$$

para todos $x, y \in G$. Logo $\psi$ é um automorfismo de $G$. Agora, defina $\rho: G \longrightarrow G$ tal que $\overline{\rho(x)}=\phi(\bar{x})$. Temos que $\rho(x)=\psi(x) \rho(1)$ para todo $x \in G$, pois

$$
\overline{\rho(x)}=\phi(\bar{x})=\phi(x) \circ \phi(\overline{1})=\psi(x) \circ \overline{\rho(1)}=\overline{\psi(x) \rho(1)} .
$$

Portanto temos que,

$$
\begin{aligned}
\psi\left(f_{1}(x y)\right) & =\phi\left(f_{1}(x y)\right) \\
& =\phi(\bar{x} * \bar{y}) \\
& =\phi(\bar{x}) \circ \phi(\bar{y}) \\
& =\overline{\rho(x)} \circ \overline{\rho(y)} \\
& =f_{2}(\rho(x) \rho(y)) \\
& =f_{2}\left(\rho(1)^{2} \psi(x y)\right) .
\end{aligned}
$$

Assim $f_{2}^{-1} \psi f_{1}(x)=\rho(1)^{2} \psi(x)$ para todo $x \in G$. Nessa última igualdade, basta fazer $x=1$ para obter $f_{2}^{-1} \psi f_{1}(1)=\rho(1)^{2}$. Reciprocamente, suponha que valha a equação 
4.2 para algum automorfismo $\psi$ de $G$ com $f_{2}^{-1} \psi f_{1}(1)=u^{2}$ para algum $u \in G$. Defina $\phi: G_{1} \longrightarrow G_{2}$ por $\phi(x)=\psi(x)$ e $\phi(\bar{x})=\overline{u \psi(x)}$. Vamos mostrar que $\phi$ é um isomorfismo. De fato,

$$
\begin{gathered}
\phi(x * y)=\phi(x y)=\psi(x y)=\psi(x) \psi(y)=\psi(x) \circ \psi(y)=\phi(x) \circ \phi(y), \\
\phi(\bar{x} * y)=\phi(\overline{x y})=\overline{u \psi(x y)}=\overline{u \psi(x) \psi(y)}=\overline{u \psi(x)} \circ \psi(y)=\phi(\bar{x}) \circ \phi(y), \\
\phi(x * \bar{y})=\phi(\overline{x y})=\overline{u \psi(x y)}=\overline{u \psi(x) \psi(y)}=\psi(x) \circ \overline{u \psi(y)}=\phi(x) \circ \phi(\bar{y})
\end{gathered}
$$

e, finalmente,

$$
\phi(\bar{x} * \bar{y})=\phi\left(f_{1}(x y)\right)=\psi\left(f_{1}(x y)\right)=f_{2}\left(u^{2} \psi(x y)\right)=\overline{u \psi(x)} \circ \overline{u \psi(y)}=\phi(\bar{x}) \circ \phi(\bar{x}) .
$$

Com isso mostramos que $\phi$ é um homomorfismo. O fato de $\psi$ ser uma bijeção de $G$, faz com que $\phi$ seja bijetora.

Se existir um automorfismo $\psi$ de $G$ tal que $f_{2}=\psi f_{1} \psi^{-1}$, diremos que as aplicações $f_{1}$ e $f_{2}$ são conjugadas em $A u t(G)$.

Corolário 4.1.7. Sejam $G$ um grupo e $f_{1}, f_{2}$ permutações de $G$ tais que $G_{1}$ e $G_{2}$ não sejam grupos. Vale que:

1. Se $f_{1}$ e $f_{2}$ são conjugadas em Aut $(G)$, então $G_{1} \simeq G_{2}$.

2. Se $f_{1}(1)=f_{2}(1)=1$, então $G_{1} \simeq G_{2}$ se e somente se $f_{1}$ e f forem conjugadas em $\operatorname{Aut}(G)$.

3. Se $f_{2} \in \operatorname{Aut}(G)$, $t$ for um quadrado em $G$ e $f_{1}(x)=f_{2}(x)$ t para todo $x \in G$, ent $\tilde{a} o G_{1} \simeq G_{2}$.

Demonstração. 1. Seja $\psi \in \operatorname{Aut}(G)$ tal que $f_{2}=\psi f_{1} \psi^{-1}$. Então $f_{2}^{-1} \psi f_{1}=\psi$ e como $f_{2}^{-1} \psi f_{1}(1)=1$ é um quadrado em $G$, segue que $G_{1} \simeq G_{2}$. 
2. Vamos supor $G_{1} \simeq G_{2}$. Pela proposição 4.1.6, existe $\psi \in \operatorname{Aut}(G)$ tal que $f_{2}^{-1} \psi f_{1}(x)=f_{2}^{-1} \psi f_{1}(1) . \psi(x)$ para todo $x \in G$ e que $f_{2}^{-1} \psi f_{1}(1)$ é um qquadrado em $G$. Como $f_{2}^{-1} \psi f_{1}(1)=1$, temos que $f_{2}^{-1} \psi f_{1}(x)=\psi(x)$ para todo $x \in G$ e assim, temos $f_{2}=\psi f_{1} \psi^{-1}$. A recíproca segue do item 1.

3. Tomando $\psi=i d_{G}$, a equação 4.2 se escreve como $f_{2}^{-1} f_{1}(x)=f_{2}^{-1} f_{1}(1) . x$. Vamos mostrar que $f_{2}^{-1} f_{1}(1)$ é um quadrado em $G$. De fato

$$
f_{2}^{-1} f_{1}(1)=f_{2}^{-1}\left(f_{2}(1) t\right)=f_{2}^{-1}(t)
$$

que, como t é um quadrado em $G$ e $f_{2} \in A u t(G)$, é um quadrado em $G$.

O que foi feito até agora neste capítulo, independe de $G(f)$ ter ou não estrutura de $A$-loop. A próxima proposição será uma ferramenta para construção de $A$-loops comutativos com núcleo intermediário de índice 2.

Proposição 4.1.8. As seguintes condições são equivalentes para um A-loop comutativo $Q$ que possui um subgrupo de indice 2.

1. $Q$ é um A-loop e $\left[Q: \mathcal{N}_{\mu}(Q)\right]=2$;

2. $Q=G(f)$ onde $G$ é um grupo abeliano, $[Q: G]=2$ e $f$ é uma permutação de $G$ satisfazendo

$$
\begin{gathered}
f(x y)=f(x) f(y) f(1)^{-1} \\
f\left(x^{2}\right)=x^{2} f(1) \\
f^{2}\left(x^{2}\right) f(x)^{-2}=f^{2}(1)
\end{gathered}
$$

para quaisquer $x, y \in G$.

3. $Q=G(f)$, onde $G$ é um grupo abeliano, $[Q: G]=2$ e $f$ é uma permutação de $G$ que satisfaz 4.3, 4.4 e $f^{2}(1)=f(1)^{2}$.

4. $Q=G(f)$, onde $G$ é um grupo abeliano, $[Q: G]=2, f(x)=g(x) t, g\left(x^{2}\right)=x^{2}$ para todo $x \in G$, onde $g \in \operatorname{Aut}(G)$ e $t$ é um ponto fixo de $g$. 
Demonstração. Vamos mostrar quue 1 é equivalente a 2, 2 é equivalente a 3 e 3 é equivalente a 4. Vamos mostrar o primeiro caso. $Q$ é um $A$-loop e $\left[Q: \mathcal{N}_{\mu}(Q)\right]=2$, se e somente se $Q=G(f)$ onde $G$ é um grupo abeliano tal que $G \leq \mathcal{N}_{\mu}(Q)$ e $f$ é uma permutação de $G$. Denote por $\alpha(a, b, c, d)$ a seguinte identidade

$$
[(a * b) \backslash(a *(b * c))] *[(a * b) \backslash(a *(b * d))]=[(a * b) \backslash(a *(b *(c * d)))],
$$

com $a, b, c, d \in G \cup \bar{G}$. Temos que $Q$ é um $A$-loop se e somente se $\alpha(a, b, c, d)$ for verdadeira para quaisquer $a, b, c, d \in Q$. Com excessão dos elementos $a, b, c, d$, os elementos sem barra estão em $G$ e os elementos sem barra são elementos de $\bar{G}$. Sejam $x, y, u, v \in G$ temos que $\alpha(x, y, u, v), \alpha(a, y, b, c)$ e $\alpha(x, \bar{y}, u, v)$ são sempre verdadeiras pois $G$ é um grupo e $G \leq \mathcal{N}_{\mu}(Q)$. Por hipótese, $Q$ é comutativo e então $\alpha(a, b, c, d)$ será verdadeira se e somente se $\alpha(a, b, d, c)$ for verdadeira. Segue da definição que:

$$
\begin{array}{r}
\alpha(x, \bar{y}, u, \bar{v}) \text { é equivalente a } f^{-1}(x f(y u v))=u f^{-1}(x f(y v)), \\
\alpha(x, \bar{y}, \bar{u}, \bar{v}) \quad \text { é o mesmo que } f(u v)=f\left((x y)^{-2} f^{-1}(x f(y u)) f^{-1}(x f(y v))\right), \\
\alpha(\bar{x}, \bar{y}, u, v) \text { é equivalente a } \quad f(x y u v)=f(x y)^{-1} f(x y u) f(x y v), \\
\alpha(\bar{x}, \bar{y}, u, \bar{v}) \quad \text { vale se e só se } \quad x f(y u v)=f(x y u) f(x y)^{-1} x f(y v),
\end{array}
$$

e finalmente temos que $\alpha(\bar{x}, \bar{y}, \bar{u}, \bar{v})$ se verifica se e somente se

$$
f(x y)^{-1} f(x y f(u v))=f\left(f(x y)^{-2} x^{2} f(y u) f(y v)\right) .
$$

Em 4.9, fazendo $x=y=1$ temos que $f(u v)=f(u) f(v) f(1)^{-1}$ para quaisquer $u, v \in G$, ou seja 4.3 se verifica. Reciprocamente se vale 4.3 então

$$
f(x y u) f(y v)=f(x y)\left(f(u) f(1)^{-1} f(y v)\right)=f(x y) f(y u v)
$$

$e$, logo, $x f(y u v)=f(x y)^{-1} f(x y u) x f(y v)$, para quaisquer $x, y, u, v \in G$. Assim a identidade $\alpha(\bar{x}, \bar{y}, u, \bar{v})$ é equivalente a equação 4.3. 
A partir de agora, vamos assumir 4.3 verdadeira e denotemos $t=f(1)$. Como 4.3 é verdadeira temos que a equação 4.7 é equivalente a

$$
x^{-1} t^{-1}=f\left(x^{-2}\right) f\left(y^{-2}\right) f(y)^{2} x t^{-5} .
$$

Como $t=f(1)=f\left(y y^{-1}\right)=f(y) f\left(y^{-1}\right) f(1)^{-1}$, segue que

$$
f\left(y^{-1}\right)=f(y)^{-1} t^{2} \quad \text { e ainda } \quad f\left(y^{-2}\right)=f(y)^{-2} t^{3} .
$$

Voltando à equação 4.11 vem que

$$
\begin{aligned}
x^{-1} t^{-1} & =f\left(x^{-2}\right) f\left(y^{-2}\right) f(y)^{2} x t^{-5} \\
& =f\left(x^{-2}\right) x t^{-2} \\
& =f\left(x^{2}\right)^{-1} x \\
& =\left(f(x)^{2} t^{-1}\right)^{-1} x=f(x)^{-2} t x .
\end{aligned}
$$

Assim $f(x)^{2}=x^{2} t$, ou equivalentemente (usando 4.3), $f\left(x^{2}\right)=x^{2} t$, que é a identidade 4.4. Agora, usando 4.3 e 4.12 segue

$$
f^{2}(u v)=f\left(f(u) f(v) t^{-1}\right)=f^{2}(u) f^{2}(v) f\left(t^{-1}\right) t^{-2}=f^{2}(u) f^{2}(v) f(t)^{-1} .
$$

A partir das equações 4.3, 4.4 e 4.13, temos que $\alpha(\bar{x}, \bar{y}, \bar{u}, \bar{v})$ é verdadeira se e só se

$$
f(t)=f(x y)^{-2} x^{2} f^{2}(y)^{2} .
$$

Nessa última equação, faça $x=1$, donde segue 4.5. Agora, assumindo 4.5 vamos mostrar 4.14. De fato

$$
\left.f(t)=f^{2}(x y) f^{2}(x y) f(x y)^{-2}=f^{(} x\right)^{2} f^{2}(y)^{2} f(x y)^{-2} f(t)^{-2} .
$$


Resta-nos mostrar que $x^{2}=f^{2}(x)^{2} f(t)^{-2}$. Bem, usando 4.3 e 4.4

$$
\begin{aligned}
f^{2}(x)^{2} f(t)^{-2} & =f(f(x)) f(f(x)) f(t)^{-2} \\
& =f\left(f(x)^{2}\right) t f(t)^{-2} \\
& =f\left(f(x)^{2}\right)\left(f(t) f(t) t^{-1}\right)^{-1} \\
& =f\left(f(x)^{2}\right) f\left(t^{2}\right)^{-1} \\
& =f\left(f(x)^{2}\right)\left(t^{2} t\right)^{-1} \\
& =f\left(x^{2}\right) t^{-2}=f\left(x^{2}\right) t^{-1} \\
& =x^{2}
\end{aligned}
$$

e assim, 4.5 é equivalente a 4.14. Finalmente, as identidades $\alpha(x, \bar{y}, \bar{v}, u), \alpha(x, y, u, \bar{v}), \alpha(\bar{x}, \bar{y}, u, v)$ seguem da identidade 4.3. Logo, 1 é equivalente a 2.

Vamos agora assumir o item 2. Basta fazer $x=1 \mathrm{em} 4.5$ para obter $f(t)=t^{2}$, o que demonstra o item 3. Agora se vale $f(t)=t^{2}$, temos

$$
f^{2}(x)^{2} f(t)^{-1}=f(f(x)) f(f(x)) t^{-2}=f\left(f(x)^{2}\right) t^{-1}=f(x)^{2},
$$

donde temos 4.5. Assim 2 é equivalente a 3.

Assuma que valha o item 3. Defina $g: G \longrightarrow G$ por $g(x)=f(x) t^{-1}$, onde $t=f(1)$.

De 4.3, temos que

$$
g(x y)=f(x y) t^{-1}=f(x) f(y) t^{-2}=f(x) t^{-1} f(y) t^{-1}=g(x) g(y)
$$

e de 4.4 temos $g\left(x^{2}\right)=f\left(x^{2}\right) t^{-1}=x^{2}$. Ainda temos que g é uma bijeção de $G$ (pois $f$ o é) e $g(t)=f(t) t^{-1} t^{2} t^{-1}=t$, donde temos o item 4. Finalmente, supondo 4, defina $f(x)=g(x) t$ onde $t$ é um ponto fixo de $g$. Temos que $f(1)=g(1) t=t$. De

$$
f(x y)=g(x y) t=g(x) t g(y) t t^{-1}=f(x) f(y) t^{-1}
$$

temos 4.3. De $f\left(x^{2}\right)=g\left(x^{2}\right) t=x^{2} t$, que é 4.4 e, por fim, $f^{2}(1)=f(t)=g(t) t=t^{2}$, provando o item 3. 


\subsection{Construções de $A$-Loops Comutativos com Núcleo Intermediário de Índice 2}

O principal objetivo dessa seção é aplicar a proposição 4.1 .8 para classificar os $A$-loops comutativos de ordem 8 e apresentar uma classe de $A$-loops comutativos de expoente 2 com centro trivial e núcleo intermediário de índice 2.

\subsubsection{A-loops comutativos de ordem 8}

Através de cálculos feitos computacionalmente, sabemos que existem $4 A$-loops não-associativos de ordem 8 , e todos esses têm núcleo intermediário de índice 2. Vamos classificar os $A$-loops comutativos de ordem 8.

Lema 4.2.1. Sejam $G$ um grupo abeliano, $g \in \operatorname{Aut}(G)$ et $\in G$. Seja $f$ a permutação de $G$ definida por $f(x)=g(x)$ t. Então $\mathcal{Z}(G(f))=\mathcal{Z}(G(g))$ como conjuntos.

Demonstração. Se $g=i d_{G}$ então tanto $g$ quanto $f$ são translações de $G$ e assim $G(g)$ e $G(g)$ são grupos abelianos. Portanto $\mathcal{Z}(G(f))=G \cup \bar{G}=\mathcal{Z}(G(g))$. Agora, se $g \neq i d_{G}$, nem $f$ nem $g$ são translações de $G$. Assim, pelo lema 4.1.3 tanto $G(f)$ como $G(g)$ são não-associativos e, além disso,

$$
\begin{gathered}
\mathcal{Z}(G(f))\{x \in G: f(x)=x f(1)\}=\{x \in G: g(x) t=x t\}= \\
=\{x \in G: g(x)=x g(1)\}=\mathcal{Z}(G(g)) . \quad
\end{gathered}
$$

Suponha $Q$ um $A$-loop comutativo de ordem $8 \operatorname{com}\left[Q: \mathcal{N}_{\mu}(Q)\right]=2$. Temos que, pela proposição 4.1.8, $Q=G(f)$ onde $G$ é um grupo abeliano de ordem 4 e $f(x)=g(x) t$ para algum $g \in \operatorname{Aut}(G)$ tal que $g\left(x^{2}\right)=x^{2}$ e $g(t)=t$. No que se segue, denotaremos os automorfismos do grupo $G$ com a notação cíclica, isto é, o automorfismo $(a, b)$ está definido por $a \mapsto b$. Vamos analisar os casos possíveis:

1. Suponha $G=\mathbb{Z}_{4}=\langle a\rangle$. O grupo $G$ possui apenas os automorfismos $(a, a)$ e $\left(a, a^{3}\right)$ e ambos fixam os quadrados de $G$. 
- Se $g=(a, a)$ temos que $f(x)=g(x) t$ é uma trasnlação e então $G(f)$ é um grupo abeliano.

- Se $g=\left(a, a^{3}\right)$ então $G(f)$ é um $A$-loop comutativo não-associativo de oredem 8. Como o único elemnto não trivial de $G$ que é fixado por $g$ é $a^{2}$, se $f(x)=g(x) a^{2}$ teremos que $G(f) \simeq G(g)$.

2. Suponha $G=\mathbb{Z}_{2} \times \mathbb{Z}_{2}=\langle a\rangle \times\langle b\rangle$. Temos que

$$
A u t(G)=\left\{i d_{G},(a, b),(a, a b),(b, a b),(a, b, a b),(b, a, a b)\right\} \simeq S_{3} .
$$

O elemento neutro 1 é o único quadrado de $G$ e é fixo por todo elemento de $\operatorname{Aut}(G)$.

- Se $g=i d_{G}$ e $f(x)=g(x) t$ para algum $t \in G$, então $G(f)$ é um grupo abeliano.

- Assuma $g_{1}=(a, b)$. A duas possíveis escolhas para $t \in G$ tal que $g(t)=t$, a saber, $t=1$ e $t=a b$. Seja $f_{1}(x)=g_{1}(x) a b$. Então $G\left(f_{1}\right)$ e $G\left(g_{1}\right)$ são $A$-loops comutativos não associativos. Afirmamos que $G\left(f_{1}\right) \nsim G\left(g_{1}\right)$. De fato, como $g_{1}(x x)=g_{1}(1)=1$, te,os que $G\left(g_{1}\right)$ tem expoente 2 , por outro lado $f_{1}(x x)=f_{1}(1)=a b$.

- Considere $g_{2}=(a, a b)$. Se $t \in G$ é tal que $g(t)=t$, então ou $t=1$ ou $t=b$. Seja $f_{2}(x)=g_{2}(x) b$. Visto que $A u t(G) \simeq S_{3}$ temos que $g_{1}$ e $g_{2}$ são conjugados em $\operatorname{Aut}(G)$, logo pelo corolário 4.1.7 temos $G\left(g_{1}\right) \simeq G\left(g_{2}\right)$. Como consequência do fato de $g_{1}$ e $g_{2}$ srem conjugados em $A u t(G)$ temos que $f_{1}$ e $f_{2}$ são conjugadas em $A u t(G)$, donde segue $G\left(f_{1}\right) \simeq G\left(f_{2}\right)$.

- Se $g_{3}=(b, a b)$ temos que $g_{3}$ e $g_{2}$ saõ conjugadas e assim $G\left(g_{3}\right)$ já foi listado anteriormente.

- Considere $g_{4}=(a, b, a b)$. Observe que o automorfismo $g_{4}$ não possui nenhum ponto fixo, exceto 1 . Como $g_{4}$ não é uma translação de $G$, então 
$G\left(g_{4}\right)$ é um $A$-loop comutativo não associativo. Pelo lema 4.1.3, segue

$$
\mathcal{Z}\left(G\left(g_{4}\right)\right)=\left\{x \in G: g_{4}(x)=x g_{4}(1)\right\}=\{1\}
$$

e

$$
\mathcal{Z}\left(G\left(g_{1}\right)\right)\left\{x \in G: g_{1}(x)=x g_{1}(1)\right\}=\{1, a b\}
$$

. Donde temos que $G\left(g_{4}\right)$ é um $A$-loop não-associativo que ainda não havia sido listado.

- Finalmente, se $g_{5}=(b, a, a b)$ então $g_{4}$ e $g_{5}$ são conjugados em $A u t(G)$ donde temos que $G\left(g_{4}\right) \simeq G\left(g_{5}\right)$.

\subsubsection{Uma classe de $A$-loops comutativos de expoente 2 com centro trivial e núcleo intermediário de índice 2}

Considere $\mathbb{F}_{2}$ o corpo com dois elementos, $V$ um $\mathbb{F}_{2}$-espaço veotorial de dimensão $n, n \geq 2$ e $G=(V,+)$ o 2-grupo abeliano elementar correspondente. Para uma base $\mathcal{B}=\left\{e_{1}, e_{2}, \ldots, e_{n}\right\}$ defina um automorfismo $g$ de $G$ fazendo

$$
g\left(e_{i}\right)=e_{i+1} \quad \text { para } \quad i=1, \ldots, n-1 \quad \text { e } \quad g\left(e_{n}\right)=e_{1}+e_{n} .
$$

Temos que $g(x+x)=g(0)=0=x+x$, então fazendo $f=g$, pela proposição 4.1.8, temos que $Q_{n}=G(f)$ é um $A$-loop comutativo de ordem $2^{n+1}$ cujo núcleo intermediário tem índice, no máximo, 2. Se

$$
g\left(a_{1} e_{1}+a_{2} e_{2}+\ldots+a_{n} e_{n}\right)=a_{1} e_{1}+a_{2} e_{2}+. .+a_{n} e_{n}
$$

então $a_{1}=a_{2}=\ldots=a_{n}=0$ o que, pelo lema 4.1.3, nos garante que $G(f)$ tem centro trivial. Segue que $\left[Q_{n}: \mathcal{N}_{\mu}\left(Q_{n}\right)\right]=2$ pois $f$ não é uma translação e como $x * x=x+x=0$ e $\bar{x} * \bar{x}=g(x+x)=0, Q_{n}$ tem expoente 2. 


\subsection{Extensões Centrais Baseadas em Formas Tri- lineares}

Nessa seção, faleremos de construções de $A$-loops comutativos através de extensões centrais de um grupo abeliano por um $A$-loop comutativo. Tais extensões serão dadas por cociclos de loops. Determinaremos condições para quando tais cociclos geram $A$ loops comutativos e grupos abelianos. Estudaremos também, cociclos definidos por formas trilineares.

Definição 4.3.1. Dizemos que o loop $Q$ é uma extensão do loop $Z$ pelo loop $K$ se $Z \triangleleft Q$ e se $Q / Z \simeq K$. No caso de $Z \leq \mathcal{Z}(Q)$, tal extensão é chamada de extensão central.

É sabido que (vide $[\mathrm{BP}]$ ) extensões centrais de um grupo abeliano $Z$ por um loop $K$ são loops da forma $K \ltimes_{\theta} Z=(Z, K, \theta(x, y))$ definidos em $K \times Z$ com

$$
(x, a)(y, b)=(x y, a b \theta(x, y))
$$

onde $\theta: K \times K \longrightarrow Z$ é uma aplicação que satisfaz $\theta(1, x)=\theta(x, 1)=1$, para todo $x \in K$. Tal aplicação $\theta$ é chamada de cociclo de loops. No teorema 2.5.3 é exibido condições para $\theta$ para que $K \ltimes_{\theta} Z$ seja um $A$-loop, onde $Z$ é um grupo abeliano e $K$ já tem estrutura de $A$-loop. Apresentaremos aqui uma versão desse teorema cuja demontração será omitida por se tratar de um caso particular da realizada no capítulo 2 .

Teorema 4.3.2. Sejam $Z$ um grupo abeliano e $K$ um A-loop comutativo. Considere $\theta: K \times K \longrightarrow Z$ satisfazendo $\theta(x, y)=\theta(y, x)$ para todo $x, y \in K e$

$$
F(x, y, z) F\left(x^{\prime}, y, z\right) \theta\left(R_{(y, z)}(x), R_{(y, z)}\left(x^{\prime}\right)\right)=F\left(x x^{\prime}, y, z\right) \theta\left(x, x^{\prime}\right)
$$

para quaisquer $x, x^{\prime}, y, z \in K$ onde a função $F$ é definida por

$F(x, y, z)=\theta\left(R_{(y, z)}(x), y z\right)^{-1} \theta(y, z)^{-1} \theta(x y, z) \theta(x, y)$. Então $K \ltimes_{\theta} Z$ é um A-loop comutativo. 
Observação 4.3.3. O teorema 4.3.2 é um caso particular do teorema 2.5.3 pois para loops comutativos $L$, o grupo das aplicações internas é gerado por $\left\{R_{(a, b)}: a, b \in L\right\}$.

Teorema 4.3.4. Sejam $Z$ um 2-grupo abeliano elementar e $K$ um A-loop comutativo de expoente 2. Considere o cociclo de loop $\theta$ tal que $\theta(x, y)=\theta(y, x)$ e $\theta(x, x)=1$ para todos $x, y \in K e$

$$
\begin{gathered}
\theta(x, y) \theta\left(x^{\prime}, y\right) \theta\left(x x^{\prime}, y\right) \theta\left(x, x^{\prime}\right) \theta(x y, z) \theta\left(x^{\prime} y, z\right) \theta(y, z) \theta\left(\left(x x^{\prime}\right) y, z\right)= \\
=\theta\left(R_{(y, z)}(x), y z\right) \theta\left(R_{(y, z,)}\left(x^{\prime}\right), y z\right) \theta\left(R_{(y, z,)}\left(x x^{\prime}\right), y z\right) \theta\left(R_{(y, z,)}(x), R_{(y, z)}\left(x^{\prime}\right)\right),
\end{gathered}
$$

para todos $x, x^{\prime}, y, z \in K$. Então $K \ltimes_{\theta} Z$ é um A-loop comutativo de expoente 2.

Demonstração. Visto que $(x, a)(x, a)=(x x, a a \theta(x, x))$, temos que $Q=K \ltimes_{\theta} Z$ é um loop comutativo de expoente 2. Resta mostrar que $Q$ é um A-loop. Sejam $(x, a),\left(x^{\prime}, a^{\prime}\right),(y, b),(z, c) \in Q . \quad$ Fazendo $R_{((y, b),(z, c))}(x, a)=(u, v)$, obtemos $u=$ $R_{(y, z)}(x)$ e $v=\theta\left(R_{(y, z)}(x), y z\right) \theta(y, z) \theta(x y, z) \theta(x, y) a$. Assim temos

$$
\begin{gathered}
R_{((y, b),(z, c))}(x, a)=\left(R_{(y, z)}(x), \theta\left(R_{(y, z)}(x), y z\right) \theta(y, z) \theta(x y, z) \theta(x, y) a\right), \\
R_{((y, b),(z, c))}\left(x^{\prime}, a^{\prime}\right)=\left(R_{(y, z)}\left(x^{\prime}\right), \theta\left(R_{(y, z)}\left(x^{\prime}\right), y z\right) \theta(y, z) \theta\left(x^{\prime} y, z\right) \theta\left(x^{\prime}, y\right) a^{\prime}\right)
\end{gathered}
$$

e também,

$$
\begin{gathered}
R_{((y, b),(z, c))}\left((x, a)\left(x^{\prime}, a^{\prime}\right)\right)=\left(R_{(y, z)}\left(x x^{\prime}\right),\right. \\
\left.\theta\left(R_{(y, z)}\left(x x^{\prime}\right), y z\right) \theta(y, z) \theta\left(\left(x x^{\prime}\right) y, z\right) \theta\left(\left(x x^{\prime}\right), y\right) a a^{\prime}\right) .
\end{gathered}
$$

Multiplicando as expressões 4.15 e 4.16 e comparando com $R_{((y, b),(z, c))}\left((x, a)\left(x^{\prime}, a^{\prime}\right)\right)$ temso que $Q$ é um A-loop.

Se tivéssimos $K$ um 2-grupo elementar a identidade do teorema 4.3.4 poderia ser escrita da forma

$$
\begin{gathered}
{\left[\theta(x, y) \theta\left(x^{\prime}, y\right) \theta\left(x x^{\prime}, y\right)\right]\left[\theta(x y, z) \theta\left(x^{\prime} y, z\right) \theta\left(x x^{\prime}, z\right)\right]} \\
{\left[\theta(x, y z) \theta\left(x^{\prime}, y z\right) \theta\left(x x^{\prime}, y z\right)\right]\left[\theta(y, z) \theta\left(x x^{\prime}, z\right) \theta\left(\left(x x^{\prime}\right) y, z\right)\right]=1 .}
\end{gathered}
$$


Por essa identidade, da vontade de impor que $\theta$ satisfaça $\theta(u, w) \theta(v, w) \theta(u v, w)=1$ para todo $u, v, w \in K$ para que o loop $Z \ltimes_{\theta} K$ se "encaixe" nas hipótese do teorema 4.3.4. Entretanto $\theta(u, w) \theta(v, w) \theta(u v, w)=1$ para todo $u, v, w \in K$ implica associatividade para $Z \ltimes_{\theta} K$, pois

$$
[(u, a)(v, b)](w, c)=(u, a)[(v, b)(w, c)]
$$

se e somente se $\theta(u v, w) \theta(u v)=\theta(u, v w) \theta(v, w)$.

Proposição 4.3.5. Seja $Z=\mathbb{F}_{2}$ e seja $K$ um 2-grupo abeliano elementar. Seja $g: K^{3} \longrightarrow \mathbb{F}_{2}$ uma forma trilinear tal que $g(x, x y, y)=g(y, x y, x)$ para todo $x, y \in K$. Defina $\theta: K^{2} \longrightarrow \mathbb{F}_{2}$ por $\theta(x, y)=g(x, x y, y)$. Então $Q=K \ltimes_{\theta} Z$ é um A-loop comutativo de expoente 2 . Além disso, $(y, b) \in \mathcal{N}_{\mu}(Q)$ se e somente se

$$
g(y, x, z)=g(x, z, y)
$$

Demonstração. Desde que $g(x, x y, y)=g(y, x y, x)$ para todos $x, y \in K$ temos $\theta(x, y)=\theta(y, x)$ para todos $x, y \in K$ e como $K$ é um 2-grupo elementar temos que $\theta(x, x)=g(x, e, x)=0$. Pela tri-linearidade de $g$, temos que

$$
\theta(u, w) \theta(v, w) \theta(u v, w)=g(u, u w, w) g(v, v w, w) g(u v, u v w, w)=g(u, v, w) g(v, u, w)
$$

donde segue que

$$
\begin{gathered}
{\left[\theta(x, y) \theta\left(x^{\prime}, y\right) \theta\left(x x^{\prime}, y\right)\right]\left[\theta(x y, z) \theta\left(x^{\prime} y, z\right) \theta\left(x x^{\prime}, z\right)\right]} \\
{\left[\theta(x, y z) \theta\left(x^{\prime}, y z\right) \theta\left(x x^{\prime}, y z\right)\right]\left[\theta(y, z) \theta\left(x x^{\prime}, z\right) \theta\left(x x^{\prime} y, z\right)\right]=1 .}
\end{gathered}
$$

Assim, pelo teorema 4.3.4, $K \ltimes_{\theta} Z$ é um A-loop comutativo de expoente 2. Finalmente, tome $(y, b) \in Q$. Por definição $(y, b) \in \mathcal{N}_{\mu}(Q)$ se e somente se

$$
[(x, a)(y, b)](z, c)=(x, a)[(y, b)(z, c)]
$$

para quaisquer $(x, a),(y, b) \in Q$ o que é equivalene a

$$
\theta(x y, z) \theta(x, y)=\theta(x, y z) \theta(y, z)
$$


para todos $x, z \in Q$ que, usando a definição de $\theta$ e a tri-linearidade de $g$, é o mesmo que $g(y, x, z)=g(x, z, y)$ para todo $x, z \in K$.

Definição 4.3.6. Seja $V=\left(\mathbb{F}_{2}\right)^{n}$. Uma forma trilinear $g: V^{3} \longrightarrow \mathbb{F}_{2}$ é chamada de (1,3)-simétrica se $g(x, y, z)=g(z, y, x)$ quaisquer que sejam $x, y, z \in V$.

Portanto uma forma trilinear (1,3)-simétrica define um cociclo de loops que gera um $A$-loop comutativo de expoente 2. Além disso $(y, b) \in \mathcal{N}_{\mu}(Q)$ se e somente se $g(y, x, z)=g(x, z, y)=g(y, z, x)$ para todos $x, z \in K$, ou seja, se a aplicação bilinear $g(y, \cdot, \cdot): V^{2} \longrightarrow \mathbb{F}_{2}$ for simétrica. Jedlicka, Kinyon, Vojtechovsky mostraram em [JKV-02] que para $n \geq 3$, e $V=\left(\mathbb{F}_{2}\right)^{n}$ sempre existe uma forma trilinear $(1,3)$ simétrica $g: V^{3} \longrightarrow \mathbb{F}_{2}$ tal que para todo $x \in V \backslash\{0\}$, a forma $g(x, \cdot, \cdot): V^{2} \longrightarrow \mathbb{F}_{2}$ não é simétrica. Além disso, se $n<3$ para qualquer forma trilinear (1,3)-simétrica $f: V^{3} \longrightarrow \mathbb{F}_{2}$ existe $y \in V, y \neq 0$ tal que $f(y, \cdot, \cdot): V^{2} \longrightarrow \mathbb{F}_{2}$ é simétrica.

Exemplo 4.3.7. Para todo $n \geq 3$, existe um A-loop $Q$ de expoente 2, de ordem $2^{n+1} \operatorname{com} \mathcal{N}_{\mu}(Q)=\mathcal{Z}(Q)$ e $|\mathcal{Z}(Q)|=2$. De fato, pela proposição 4.3 .5 e pelo fato de que existe $g: V^{3} \longrightarrow \mathbb{F}_{2}$ trilinear (1,3)-simétrica, onde $V=\left(\mathbb{F}_{2}\right)^{n}$, tal que se $g(x, \cdot, \cdot): V^{2} \longrightarrow \mathbb{F}_{2}$ for simétrica, então $x=0$, temos que $Q=V \ltimes_{\theta} \mathbb{F}_{2}$, onde $\theta(x, y)=g(x, x y, y)$ é um A-loop. Sabemos que $\left(0, \mathbb{F}_{2}\right)$ é um subloop normal de $Q$ contido em $\mathcal{Z}(Q)$. Por outro lado, se $(x, a) \in \mathcal{Z}(Q)$, em particular, $(x, a) \in \mathcal{N}_{\mu}(Q)$ e então, $g(x, \cdot, \cdot): V^{2} \longrightarrow \mathbb{F}_{2}$ é simétrica, e assim $x=0$. Logo $\mathcal{N}_{\mu}(Q)=\mathcal{Z}(Q)=\left(0, \mathbb{F}_{2}\right)$ $e|\mathcal{Z}(Q)|=2$.

Vimos que se $Q$ for um $A$-loop comutativo finito de expoente $2,|Q|=2^{k}$ e $\left|\mathcal{N}_{\mu}(Q)\right|=2^{l}$ onde $l \leq k$. Queremos determinar todos os possíveis pares $(k, l)$ com $l>0$.

Lema 4.3.8. Seja $k \geq l>0$. Então existe um A-loop comutativo não associativo de ordem $2^{k}$ com núcleo intermediário de ordem $2^{l}$ se ou $d=k-l \geq 3$ ou $d \geq 1$ e $l \geq 2$.

Demonstração. Suponha $d \geq 3$.

Considere o A-loop $Q$ de ordem $2^{d+1} \operatorname{com}\left|\mathcal{N}_{\mu}(Q)\right|=2$. Se $k-d=l=1$, Q é o loop 
procurado. Caso $k-d=l>1$, tome $L=Q \times\left(\mathbb{Z}_{2}\right)^{k-d-1}$. Claramente $L$ é um A-loop não associativo de ordem $2^{k}$ e como $\mathcal{N}_{\mu}(L)=\mathcal{N}_{\mu}(Q) \times\left(\mathbb{Z}_{2}\right)^{k-d-1}$, temos que $\left|\mathcal{N}_{\mu}(L)\right|=$ $2^{l}$. Agora assuma $d=2$. Se $l=1$, temos o parâmetro $(3,1)$. Pela seção anterior, todo A-loop comutativo não associativo de ordem 8, possui núcleo intermediário de indice 2, portanto de ordem 4. Jedlicka, Kinyon e Vojtechovsky, citam em [JKV-02], que, computacionalmente, é possível encontrar um A-loop comutativo L, de ordem 16 com $\left|\mathcal{N}_{\mu}(L)\right|=4$. Finalmente para um par de parâmetros $(k, l)$ com $l>2$ e $k-l=2$ faça $Q=L \times\left(\mathbb{Z}_{2}\right)^{k-4}$. O caso $d=1$ está feito na seção, 4.2.2, onde, para $Q$ ser não associativo, devemos ter $l>1$.

\subsubsection{Somando Cociclos de Grupos}

Dizemos que o cociclo de loops $\theta: K \times K \longrightarrow Z$, onde $K$ é um loop e $Z$ é um grupo abeliano é um cociclo de grupos se $\theta$ satisfizer a identidade

$$
\theta(x, y) \theta(x y, z)=\theta(y, z) \theta(x, y z)
$$

para todos $x, y, z \in K$. O nome cociclo de grupos é justificado pelo fato de que se $K$ for um grupo e $\theta$ um cociclo de grupos, então $K \ltimes_{\theta} Z$ também será um grupo.

Lema 4.3.9. Sejam $Z$ um grupo abeliano, $K$ um grupo e $\theta, \mu: K \times K \longrightarrow Z$ cociclos de loops tais que $\nu=\theta \mu^{-1}:(x, y) \mapsto \theta(x, y) \mu(x, y)^{-1}$ seja um cociclo de grupo. Então as aplicações internas à esquerda $\left(L_{(\alpha, \beta)}\right)$ em $K \ltimes_{\theta} Z$ coincidem com as em $K \ltimes_{\mu} Z$.

Demonstração. Em $K \ltimes_{\theta} Z$ vale que

$$
(x, a)(y, b)=(x y, \theta(x, y) a b) \quad e \quad(x, a) \backslash(y, b)=\left(x \backslash y, a^{-1} b \theta(x, x \backslash y)^{-1}\right) .
$$

Assim, temos que

$$
[(x, a)(y, b)] \backslash(x, a)[(y, b)(z, c)]=\left(z, \theta(x, y)^{-1} \theta(x, y z) \theta(y, z) \theta(x y, z)^{-1} c\right) .
$$

Então as aplicações internas à esquerda de $K \ltimes_{\theta} Z$ e $K \ltimes_{\mu} Z$ coincidem se e somente se

$$
\theta(x, y)^{-1} \theta(x, y z) \theta(y, z) \theta(x y, z)^{-1}=\mu(x, y)^{-1} \mu(x, y z) \mu(y, z) \mu(x y, z)^{-1}
$$


para todo $x, y, z \in K$, ou seja se $\nu$ for um cociclo de grupos.

Lema 4.3.10. Sejam $Z$ um grupo abeliano, $K$ um grupo e $\theta$ um cociclo de loops tal que $K \ltimes_{\theta} Z$ seja um A-loop comutativo. Seja $\mu: K \times K \longrightarrow Z$ um cociclo de grupos satisfazendo $\mu(x, y)=\mu(y, x)$ para todos $x, y \in K$. Então $K \ltimes_{\mu \theta} Z$ é um A-loop comutativo com as mesmas aplicações internas à esquerda de $K \ltimes_{\theta} Z$

Demonstração. Denotemos $Q_{\theta}=K \ltimes_{\theta} Z$ e $Q_{\mu \theta}=K \ltimes_{\mu \theta} Z$. Visto que $Q_{\theta}$ é comutativo e $\mu(x, y)=\mu(y, x)$, temos que $Q_{\mu \theta}$ também é comutativo. Como $\mu=$ $(\mu \theta) \theta^{-1}$ é um cociclo de grupo, pelo lema 4.17 as aplicações internas à esquerda de $Q_{\theta}$ e $Q_{\mu \theta}$ coincidem. Resta mostrar que $\mathcal{I}\left(Q_{\mu \theta}\right) \leq \operatorname{Aut}\left(Q_{\mu \theta}\right)$. Antes disso, obeserve que, se cdot denota a multiplicação de $Q_{\theta}$ e* a de $Q_{\mu \theta}$, temos

$$
\begin{gathered}
(x, a) *(y, b)=(x y, \mu(x, y) \theta(x, y) a b)=(x y, \theta(x, y) a b) \cdot(1, \mu(x, y))= \\
=(x, a) \cdot(y, b) \cdot(1, \mu(x, y)) .
\end{gathered}
$$

Seja $\varphi$ uma aplicação interna à esquerda de $Q_{\mu \theta}$, então

$$
\begin{aligned}
\varphi((x, a) *(y, b)) & =\varphi((x, a) \cdot(y, b) \cdot(1, \mu(x, y)) \\
& =\varphi(x, a) \cdot \varphi(y, x) \cdot(1, \mu(x, y)) \\
& =\left(x, a^{\prime}\right) \cdot\left(y, b^{\prime}\right) \cdot(1, \mu(x, y)) \\
& =\left(x, a^{\prime}\right) *\left(y, b^{\prime}\right) \\
& =\varphi(x, a) * \varphi(y, b),
\end{aligned}
$$

onde 4.18 é veradeira pois $(1, \mu(x, y)) \in \mathcal{Z}\left(Q_{\theta}\right)$ e 4.19 vale porque $Q_{\theta}$ é um A-loop. $\operatorname{Logo}, \varphi \in \operatorname{Aut}\left(Q_{\mu \theta}\right)$.

\subsection{Uma Classe de $A$-Loops Comutativos de Or- $\operatorname{dem} p^{3}$}

Encerramos esse trabalho construindo um classe de $A$-loops comutativos finitos de ordem $p^{3}$. 
Seja $Q$ um $A$-loop comutativo finito de ordem ímpar. Consideremos (vide capítulo 3) o loop $(Q, \circ)$ onde

$$
x \circ y=\left(x^{-1} \backslash x y^{2}\right)^{\frac{1}{2}} \quad \text { para } \quad x, y \in Q .
$$

Sabemos que o loop $(Q, \circ)$ é um loop de Bruck e além disso sabemos que $(Q, \circ)$ será comutativo se e somente se for isomorfo a $Q$.

Proposição 4.4.1. Seja p um primo ímpar e seja $Q$ um A-loop comutativo de ordem $p, 2 p, 4 p, p^{2}, 2 p^{2}$ e $4 p^{2}$. Então, $Q$ é um grupo abeliano.

Demonstração. Como A-loops de ordem menor que ou igual a 5 são grupos abelianos, se mostrarmos o resultado para A-loops de ordem p e $p^{2}$, do Teorema da Decomposição obteremos os outros casos. Caso $|Q|=p$, temos que $Q$ é o grupo cíclico de ordem p pois vale o Teorema de Lagrange em $Q$ e $Q$ associa potências. Caso $|Q|=p^{2}$, $Q$ é um grupo pois Burn em Finite Bol Loops, Math. Proc. Cambriage Philos. Soc., vol 84 (1978) n.03 377 - 385 mostrou que todo loop de Bol de ordem pa é um grupo. $\operatorname{Assim}(Q, \circ)$ é um grupo de ordem $p^{2}$, portanto abeliano. Logo $(Q, \circ)$ é isomorfo a Qe assim $Q$ é um grupo abeliano.

A partir de agora, estudaremos alguns $A$-loops de ordem $p^{3}$ para $p$ um primo ímpar (Tratamos de $A$-loops de ordem 8, no início desse capítulo).

Lema 4.4.2. Não existe A-loop comutativo cujo centro tem ídice p.

Demonstração. Vamos supor que $Q$ seja um A-loop comutativo tal que $|Q / \mathcal{Z}(Q)|=$ p, para algum primo p. Então, pelo Teorema de Lagrange e porque A-loops associam potências, temos que $Q / \mathcal{Z}(Q)$ é o grupo cíclico de ordem $p$. Se $x \mathcal{Z}(Q) \in$ $Q / \mathcal{Z}(Q) \backslash\{1\}$, todo elemento de $Q$ pode ser escrito da forma $x^{i} z$ com $i=1, . ., p-1$ e $z \in \mathcal{Z}(Q)$. Suponha $0 \leq i, j, k<p$ e $z_{1}, z_{2}, z_{3} \in \mathcal{Z}(Q)$, então

$$
\left(x^{i} z_{1} \cdot x^{j} z_{2}\right) x^{k} z_{3}=\left(x^{i} x^{j}\right) x^{k} \cdot z_{1} z_{2} z_{3}=x^{i} z_{1}\left(x^{j} z_{2} \cdot x^{k} z_{3}\right)
$$

Assim $Q$ é um grupo abeliano cujo centro tem índice $p$, o que é uma contradição. 
Como corolário desse lema temos que $A$-loops finitos, comutativos, não associativos e de ordem $p^{3}$, têm centro de ordem 1 ou $p$. Se $p$ for ímpar, o centro de $Q$ tem ordem $p$.

Definição 4.4.3. Seja $n \geq 1$. definimos o "overflow indicator"pela função $(\cdot, \cdot)_{n}: \mathbb{Z}_{n} \times \mathbb{Z}_{n} \longrightarrow\{0,1\}$ dada por

$$
(x, y)_{n}= \begin{cases}1, & x+y \geq n \\ 0, & x+y<n\end{cases}
$$

Denotando por $\oplus$ a soma em $\mathbb{Z}_{n}$ e + a em $\mathbb{Z}$ temos que para quaisquer $x, y \in$ $\mathbb{Z}_{n}$, vale $x \oplus y=x+y-n(x, y)_{n}$ e assim

$$
(x, y)_{n}=\frac{x+y-(x \oplus y)}{n} .
$$

Note que da eqaução 4.21 segue

$$
(x, y)_{n}+(x \oplus y, z)_{n}=(y, z)_{n}+(x, y \oplus z)_{n} \quad \text { para quaisquer } \quad x, y, z \in \mathbb{Z}_{n}
$$

A partir de agora, não vamos mais fazer diferença entre os sinais de soma de $\mathbb{Z}$ e $\mathbb{Z}_{n}$.

Definição 4.4.4. Para $n \geq 1$ e $a, b \in \mathbb{Z}_{n}$, defina $\mathcal{Q}_{a, b}\left(\mathbb{Z}_{n}\right)$, em $\mathbb{Z}_{n} \times \mathbb{Z}_{n} \times \mathbb{Z}_{n}$ por

$$
x y=\left(x_{1}+y_{1}+\left(x_{2}+y_{2}\right) x_{3} y_{3}+a\left(x_{2}, y_{2}\right)_{n}+b\left(x_{3}+y_{3}\right)_{n}, x_{2}+y_{2}, x_{3}+y_{3}\right) .
$$

onde $x=\left(x_{1}, x_{2}, x_{3}\right)$ e $y=\left(y_{1}, y_{2}, y_{3}\right) \in \mathcal{Q}_{a, b}\left(\mathbb{Z}_{n}\right)$.

Temos que $\mathcal{Q}_{a, b}\left(\mathbb{Z}_{n}\right)$ é um loop comutativo com elemnto neutro $(0,0,0)$ de ordem $n^{3}$, pois este pode ser visto como extensão de $\mathbb{Z}_{n}$ por $\mathbb{Z}_{n} \times \mathbb{Z}_{n}$ via o cociclo de loops

$$
\theta\left(\left(x_{2}, x_{3}\right),\left(y_{2}, y_{3}\right)\right)=\left(x_{2}+y_{2}\right) x_{3} y_{3}+a\left(x_{2}, y_{2}\right)_{n}+b\left(x_{3}, y_{3}\right)_{n}
$$

Podemos escrever o cociclo $\theta$ como a soma de cociclos $\theta=\mu+\nu$, onde $\mu\left(\left(x_{2}, x_{3}\right),\left(y_{2}, y_{3}\right)\right)=\left(x_{2}+y_{2}\right) x_{3} y_{3}$ e $\nu\left(\left(x_{2}, x_{3}\right),\left(y_{2}, y_{3}\right)\right)=a\left(x_{2}, y_{2}\right)_{n}+b\left(x_{3}, y_{3}\right)_{n}$, e note que, da equação 4.22, temos que $\nu$ é um cociclo de grupos. 
O objetivo é estudar exemplos de $A$-loops comutativos de ordem $p^{3}$, para $p$, um primo ímpar, dado por $\mathcal{Q}_{a, b}\left(\mathbb{Z}_{p}\right)$. Temos em [JKV-02], a seguinte proposição, que explicita algumas identidades de $\mathcal{Q}_{a, b}\left(\mathbb{Z}_{p}\right)$ e será útil na demonstração dos próximos resultados.

Proposição 4.4.5. Sejam $n \geq 2$ e $a, b \in \mathbb{Z}_{n}$. Para $x=\left(x_{1}, x_{2}, x_{3}\right), y=\left(y_{1}, y_{2}, y_{3}\right) e$ $z=\left(z_{1}, z_{2}, z_{3}\right) \in Q=\mathcal{Q}_{a, b}\left(\mathbb{Z}_{n}\right)$, valem:

1. $x \backslash y=\left(y_{1}-x_{1}-\left(y_{3}-x_{3}\right) x_{3} y_{2}-a\left(x_{2}, x_{2}-y_{2}\right)_{n}-b\left(x_{3}, y_{3}-x_{3}\right)_{n}, y_{2}-x_{2}, y_{3}-x_{3}\right)$;

2. $(x y) \backslash x(y z)=\left(z_{1}+y_{3}\left(x_{3} z_{2}-x_{2} z_{3}\right), z_{2}, z_{3}\right)$;

3. $Q$ é um A-loop não associativo, comutativo de ordem $n^{3}$;

4. $\mathcal{N}_{\lambda}(Q)=\mathcal{Z}(Q)=\mathbb{Z}_{n} \times 0 \times 0$ e $\mathcal{N}_{\mu}(Q)=\mathbb{Z}_{n} \times \mathbb{Z}_{n} \times 0$, como subconjuntos de $Q$;

5. $Q / \mathcal{Z}(Q) \simeq \mathcal{I}(Q)$ e $\mathcal{I}(Q)=\left\{L_{(v, v)}: u, v \in Q\right\}$

6. Para todo $m \geq 0$,

$$
x^{m}=\left(m x_{1}+2\left(\begin{array}{c}
m+1 \\
3
\end{array}\right) x_{2} x_{3}^{2}+a t_{2}+b t_{3}, m x_{2}, m x_{3}\right),
$$

onde $t_{i}=\sum_{k=1}^{m-1}\left(x_{i}, k x_{i}\right)_{n}$.

As somas são consideradas vazias e o coeficiente binomial nulo se $m<2$.

Lema 4.4.6. Sejam $p$ um número primo $a, b \in \mathbb{Z}_{p}$ e $Q=\mathcal{Q}_{a, b}\left(\mathbb{Z}_{p}\right)$. Então:

1. se $(a, b)=(0,0)$ e $p \neq 3$, então $Q$ tem expoente $p$;

2. $(a, b) \neq(0,0)$ ou $p=3$, então $Q$ tem expoente $p^{2}$;

3. Se $a=0$ ent $\tilde{a} o \mathcal{N}_{\mu}(Q) \simeq \mathbb{Z}_{p} \times \mathbb{Z}_{p}$;

4. Se $a \neq 0$ então $\mathcal{N}_{\mu}(Q) \simeq \mathbb{Z}_{p^{2}}$. 
Demonstração. Como $|Q|=p^{3}$ todo elemento de $Q$ têm ordem potência de $p$, então expoente de $Q$ é $p, p^{2}$ ou $p^{3}$. Visto que $Q$ não é associativo, então $Q$ não pode ter expoente $p^{3}$, pois caso contrário, $Q$ seria o grupo cíclico de ordem $p^{3}$.

1. Assuma $a=b=0$. Pela proposição 4.4.5 temos que

$\left(x_{1}, x_{2}, x_{3}\right)^{p}=\left(2\left(\begin{array}{c}p+1 \\ 3\end{array}\right) x_{2} x_{3}^{2}, 0,0\right)$. Mas o inteiro $2\left(\begin{array}{c}p+1 \\ 3\end{array}\right)$ é divisivel por $p$ se $e$ somente se $p \neq 3$. Assim, $Q$ tem expoente 2 .

2. É suficinte mostrar que $Q$ tem expoente $p^{2}$ se $(a, b) \neq(0,0)$. Primeiro, assuma $a \neq 0$. Então $(0,1,0)^{p}=(a, 0,0) \neq 0$ pois $\sum_{k=1}^{p-1}(1, k)_{p}=1$. Da mesma forma se $b \neq 0$, então $(0,0,1)^{p}=(b, 0,0) \neq 0$ e isso mostra que $Q$ não pode ter expoente $p$.

3. Se $a=0$, temos $\left(x_{1}, x_{2}, 0\right)^{p}=(0,0,0) \neq 0$, o que mostra que $\mathcal{N}_{\mu}(Q) \simeq \mathbb{Z}_{p} \times \mathbb{Z}_{p}$.

4. Se $a \neq 0$, temos que $(0,1,0)^{p}=(a, 0,0) \neq 0$, ou seja $\mathcal{N}_{\mu}(Q)$ é um grupo abeliano em um loop de expoente $p^{2}$, com um elemento não nulo que não possui ordem p. $\operatorname{Logo}, \mathcal{N}_{\mu}(Q) \simeq \mathbb{Z}_{2}$.

No próximo lema, mostraremos que uma condição suficiente para $a_{1}, a_{2} \in \mathbb{Z}_{p}$, onde $p$ é ímpar, para que os loops $\mathcal{Q}_{a_{1}, 0}$ e $\mathcal{Q}_{a_{2}, 0}$ sejam isomorfos. Na verdade é suficiente que $a_{1} a_{2}^{-1}$ seja um resíduo quadrático de $p$. Usando os símbolos de Legendre, basta que $a_{1}$ e $a_{2}$ sejam, simultaneamente resíduos quadráticos de $p$ ou simultaneamente resíduos não quadráticos. Lembrando que $a \in \mathbb{Z}_{p}$ é um resíduo quadrático de $p$ se a equação $x^{2} \equiv a(\bmod p)$ tem solução.

Lema 4.4.7. Sejam p um primo impar e $a_{1}, a_{2} \in \mathbb{Z}_{p}^{*}$. Se $a_{1}$ e $a_{2}$ são ambos resíduos quadráticos ou ambos resíduos não quadáticos, então $\mathcal{Q}_{a_{1}, 0} \simeq \mathcal{Q}_{a_{2}, 0}$.

Demonstração. Sendo $a_{1} a_{2}^{-1}$ resíduo quadrático de $p$, existe $u \in \mathbb{Z}_{p}^{*}$ tal que $a_{2}=$ $a_{1} u^{2}$. Definindo $\varphi: \mathcal{Q}_{a_{1}, 0} \longrightarrow \mathcal{Q}_{a_{2}, 0}$ por $\varphi\left(x_{1}, x_{2}, x_{3}\right)=\left(u^{2} x_{1}, x_{2}, u x_{3}\right)$. Desde que $u \in \mathbb{Z}_{p}^{*}$ temos que $\varphi$ é uma bijeção. Vamos mostrar que $\varphi$ é um isomorfismo. Temos 
que

$$
\begin{gathered}
\varphi\left(\left(x_{1}, x_{2}, x_{3}\right)\left(y_{1}, y_{2}, y_{3}\right)\right)=\varphi\left(x_{1}+y_{1}+\left(x_{2}+y_{2}\right) x_{3} y_{3}+a_{1}\left(x_{2}, y_{2}\right)_{p}, x_{2}+y_{2}, x_{3}+y_{3}\right)= \\
=\left(u^{2}\left(x_{1}+y_{1}\right)+u^{2}\left(x_{2}+y_{2}\right) x_{3} y_{3}+a_{1} u^{2}\left(x_{2}, y_{2}\right)_{p}, x_{2}+y_{2}, u\left(x_{3}+y_{3}\right)\right) .
\end{gathered}
$$

Por outro lado,

$$
\begin{gathered}
\varphi\left(x_{1}, x_{2}, x_{3}\right) \varphi\left(y_{1}, y_{2}, y_{3}\right)=\left(u^{2} x_{1}, x_{2}, u x_{3}\right)\left(u^{2} y_{1}, y_{2}, u y_{3}\right)= \\
=\left(u^{2}\left(x_{1}+y_{1}\right)+u^{2}\left(x_{2}+y_{2}\right) x_{3} y_{3}+a_{2}\left(x_{2}, y_{2}\right)_{p}, x_{2}+y_{2}, u\left(x_{3}+y_{3}\right)\right) .
\end{gathered}
$$

Donde segue que $\varphi$ é um homomorfismo.

Apresentamos aqui uma forma de construir $A$-loops comutativos de ordem $p^{3}$. Jedlicka, Kinyon, Vojtechovsky cojecturam em [JKV-02], uma "versão dual"para o lema 4.4.7 que diz que, se $p>3, a_{1} \in \mathbb{Z}_{p}^{*}$ for resíduo quadrático de $p$ e $a_{2} \in \mathbb{Z}_{p}^{*}$ não o for, então $\mathcal{Q}_{a_{1}, 0}$ não é isomorfo a $\mathcal{Q}_{a_{2}, 0}$. 


\section{Capítulo 5}

\section{Apêndice: $A$-Loops Comutativos Nilpotentes de Grau 2}

Grichkov e Vojtechovsky estão estudando $A$-loops comutativos nilpotentes de grau 2. Tais loops têm a propriedade de qualquer associador se um elemento central. Em outras palavras o $A$-loop comutativo $L$ é tal que

$$
A(L)=\{(x, y, z): x, y, z \in L\} \subset \mathcal{Z}(L)
$$

onde $(x, y, z)$ é o único elemento de $L$ que satisfaz a equação $(x y) z=[x(y z)](x, y, z)$. Um dos principais objetivos do estudo deles é demonstrar o seguinte teorema:

Teorema 5.0.8. Sejam $X=\left\{x_{1}, x_{2}, \ldots\right\}$ um conjunto enumerável, $Y=\left\{\left(x_{i}, x_{j}, x_{k}\right)\right.$ : $\left.x_{i}, x_{j}, x_{k} \in X, i<k\right\}$ e $A$ e $B$ grupos abelianos livres gerados por $X$ e $Y$, respectivamente. Então $F=A \times B$ admite uma estrutura de A-loop comutativo nilpotente de grau 2, tal que

$$
\mathcal{N}_{\mu}(F)=\mathcal{N}_{\rho}(L)=\mathcal{N}_{\lambda}(L)=B .
$$

Mais ainda $\left(x_{i}, x_{j}, x_{k}\right)=\left(x_{i}, x_{j}, x_{k}\right) \in Y$ se $i<k$.

Nesse apêndice, estudaremos um caso particular, onde $X$ possui dois elementos. Para simplificar a notação, vamos escrever $x y . z$ para indicar $(x y) z$. Seja $L$ um 
A-loop comutativo. Em [BP] vemos que $L=G \ltimes_{\theta} Z$, onde $Z \subset \mathcal{Z}(L), L / Z=G$ é um grupo abeliano e $\theta: G \times G \rightarrow Z$ é um cociclo de loops. Sejam $g_{1}, g_{2} \in G$ e $k_{1}, k_{2} \in Z$. Notemos que

$$
\left(g_{1}, k_{1}\right)\left(g_{2}, k_{2}\right)=\left(g_{1} g_{2}, k_{1} k_{2} \theta\left(g_{1}, g_{2}\right)\right)
$$

Vamos denotar por $g$ o elemento $(g, 1) \in L$. De $g_{1} g_{2} . g_{3}=\left(g_{1} . g_{2} g_{3}\right)\left(g_{1}, g_{2}, g_{3}\right)$ tiramos que

$$
\left(g_{1}, g_{2}, g_{3}\right)=\left(1, \theta\left(g_{1}, g_{2}\right) \theta\left(g_{1} g_{2}, g_{3}\right) \theta\left(g_{2}, g_{3}\right)^{-1} \theta\left(g_{1}, g_{2} g_{3}\right)^{-1}\right)
$$

que escreveremos apenas como

$$
\left(g_{1}, g_{2}, g_{3}\right)=\theta\left(g_{1}, g_{2}\right) \theta\left(g_{1} g_{2}, g_{3}\right) \theta\left(g_{2}, g_{3}\right)^{-1} \theta\left(g_{1}, g_{2} g_{3}\right)^{-1}
$$

Para $g_{1}, g_{2}, g_{3}, g_{4} \in G$ vale que

$$
\begin{aligned}
g_{1} g_{2} . g_{3} g_{4} & =g_{1}\left(g_{2} . g_{3} g_{4}\right)\left(g_{1}, g_{2}, g_{3} g_{4}\right) \\
& =g_{1}\left(g_{3} . g_{2} g_{4}\right)\left(g_{3}, g_{4}, g_{2}\right)\left(g_{1}, g_{2}, g_{3} g_{4}\right) \\
& =\left(g_{2} g_{4} . g_{3}\right) g_{1}\left(g_{3}, g_{4}, g_{2}\right)\left(g_{1}, g_{2}, g_{3} g_{4}\right) \\
& =\left(g_{1} g_{3} . g_{2} g_{4}\right)\left(g_{2} g_{4}, g_{3}, g_{1}\right)\left(g_{3}, g_{4}, g_{2}\right)\left(g_{1}, g_{2}, g_{3} g_{4}\right),
\end{aligned}
$$

e assim, por 5.1, temos

$$
\theta\left(g_{1} g_{2}, g_{3} g_{4}\right)=\theta\left(g_{1} g_{3}, g_{2} g_{4}\right)\left(g_{4} g_{2}, g_{3}, g_{1}\right)\left(g_{3}, g_{4}, g_{2}\right)\left(g_{1}, g_{2}, g_{3} g_{4}\right)
$$

Da mesma forma obtemos

$$
\begin{gathered}
\theta\left(g_{1} g_{2}, g_{3} g_{4}\right)=\theta\left(g_{1} g_{3}, g_{2} g_{4}\right)\left(g_{1} g_{3}, g_{2}, g_{4}\right)\left(g_{2}, g_{1}, g_{3}\right)\left(g_{4}, g_{3}, g_{1} g_{2}\right) \quad \text { e } \\
\theta\left(g_{1} g_{2}, g_{3} g_{4}\right)=\theta\left(g_{1} g_{3}, g_{2} g_{4}\right)\left(g_{1} g_{3}, g_{4}, g_{2}\right)\left(g_{4}, g_{3}, g_{1}\right)\left(g_{2}, g_{1}, g_{3} g_{4}\right)
\end{gathered}
$$

Note que, para quaisquer elementos $a, b, x, y$ no $A$-loop comutativo $L,(a b . x) y=$ $(a b . x y)(a b, x, y)$ e, visto que, $R_{(x, y)}(a) R_{(x, y)}(b)=R_{(x, y)}(a b)$, temos que $(a b, x, y)=$ $(a, x, y)(b, x, y)$. Ainda

$a x . b=(a . x b)(a, x, b)=(b x . a)(a, x, b)=(b . x a)(b, x, a)(a, x, b)=(a x . b)(b, x, a)(a, x, b)$, 
que implica que $(a, x, b)=(b, x, a)^{-1}$, e também

$$
a x . a=(a . x a)(a, x, a)=(a . x a)(a, x, a) .
$$

Resumindo, para quaisquer $a, b, x, y \in L$, temos

$$
(a b, x, y)=(a, x, y)(b, x, y), \quad(a, x, b)=(b, x, a)^{-1} \quad \text { e } \quad(a, x, a)=1 .
$$

Como dito antes, vamos supor

$$
X=\left\{x_{1}, x_{2}\right\} \quad \text { e } \quad Y=\left\{z_{1}=\left(x_{1}, x_{1}, x_{2}\right), z_{2}=\left(x_{1}, x_{2}, x_{2}\right)\right\}
$$

Sejam $g_{1}=x_{1}^{i}, g_{2}=x_{2}^{s}, g_{3}=x_{1}^{j}, g_{4}=x_{1}^{t} \in L$. Como $L$ associa potências, segue que $\theta\left(x_{k}^{l}, x_{k}^{m}\right)=1$ com $k=1,2$ e $l, m \in \mathbb{Z}$. Das equações 5.3 , 5.4 e 5.5, temos que

$$
\begin{aligned}
\left(x_{2}^{s+t}, x_{1}^{j}, x_{1}^{i}\right)\left(x_{1}^{j}, x_{2}^{t}, x_{2}^{s}\right)\left(x_{1}^{i}, x_{2}^{s}, x_{1}^{j} x_{2}^{t}\right) & =\left(x_{1}^{i+j}, x_{2}^{s}, x_{2}^{t}\right)\left(x_{2}^{t}, x_{1}^{j}, x_{1}^{i} x_{2}^{s}\right)\left(x_{2}^{s}, x_{1}^{i}, x_{1}^{j}\right) \\
& =\left(x_{1}^{i+j}, x_{2}^{t}, x_{2}^{s}\right)\left(x_{2}^{s}, x_{1}^{i}, x_{1}^{j} x_{2}^{t}\right)\left(x_{2}^{t}, x_{1}^{j}, x_{1}^{i}\right)
\end{aligned}
$$

Agora, por 5.6, temos

$$
\left(x_{1}, x_{2}^{s}, x_{2}\right)^{t(i+j)}=\left(x_{1}, x_{2}^{t}, x_{2}\right)^{s(i+j)}
$$

ou seja

$$
\left(x_{1}, x_{2}^{s}, x_{2}\right)^{t}=\left(x_{1}, x_{2}^{t}, x_{2}\right)^{s}
$$

Fazendo $t=1$ temos

$$
\left(x_{1}, x_{2}^{s}, x_{2}\right)=\left(x_{1}, x_{2}, x_{2}\right)^{s}
$$

Sejam $g_{1}, g_{2}, g_{3}, g_{4}, g_{5}, g_{6} \in G$. Da equação 5.2 segue que

$$
\left(g_{1} g_{2}, g_{3} g_{4}, g_{5} g_{6}\right)=\theta\left(g_{1} g_{2}, g_{3} g_{4}\right) \theta\left(g_{3} g_{4}, g_{5} g_{6}\right)^{-1} \theta\left(g_{1} g_{3} . g_{2} g_{4}, g_{5} g_{6}\right) \theta\left(g_{1} g_{2}, g_{3} g_{5} . g_{4} g_{6}\right)^{-1}
$$

e assim, por 5.3 e 5.6 temos

$$
\left(g_{1} g_{2}, g_{3} g_{4}, g_{5} g_{6}\right)=\theta\left(g_{1} g_{3}, g_{2} g_{4}\right)\left(g_{2} g_{4}, g_{3}, g_{1}\right)\left(g_{3}, g_{4}, g_{2}\right)\left(g_{1}, g_{2}, g_{3}\right)\left(g_{1}, g_{2}, g_{4}\right)
$$




$$
\begin{gathered}
\theta\left(g_{3} g_{5}, g_{4} g_{6}\right)^{-1}\left(g_{4} g_{6}, g_{5}, g_{3}\right)^{-1}\left(g_{5}, g_{6}, g_{4}\right)^{-1}\left(g_{3}, g_{4}, g_{5}\right)^{-1}\left(g_{3}, g_{4}, g_{6}\right)^{-1} \\
\theta\left(g_{1} g_{3} g_{5}, g_{2} g_{4} g_{6}\right)\left(g_{2} g_{4} g_{6}, g_{5}, g_{1} g_{3}\right)\left(g_{5}, g_{6}, g_{2} g_{4}\right)\left(g_{1} g_{3}, g_{2} g_{4}, g_{5}\right)\left(g_{1} g_{3}, g_{2} g_{4}, g_{6}\right) \\
\theta\left(g_{1} g_{3} g_{5}, g_{2} g_{4} g_{6}\right)^{-1}\left(g_{2} g_{4} g_{6}, g_{3} g_{5}, g_{1}\right)^{-1}\left(g_{3} g_{5}, g_{4} g_{6}, g_{2}\right)^{-1}\left(g_{1}, g_{2}, g_{3} g_{5}\right)^{-1}\left(g_{1}, g_{2}, g_{4} g_{6}\right)^{-1} \text {. }
\end{gathered}
$$

Note que para $\left(x_{1}^{m}, w_{1}\right),\left(x_{2}^{l}, w_{2}\right) \in L=G \ltimes_{\theta} Z$,

$$
\left(x_{1}^{m}, w_{1}\right)\left(x_{2}^{s}, w_{2}\right)=\left(x_{1}^{m} x_{2}^{l}, w_{1} w_{2} \theta\left(x_{1}^{m}, x_{2}^{l}\right)\right)
$$

Nessa última igualdade, fazendo $w_{1}=w_{2}=1$, temos $\theta\left(x_{1}^{m}, x_{2}^{l}\right)=1$.

Agora, substituindo $g_{1}=x_{1}^{i}, g_{2}=x_{2}^{s}, g_{3}=x_{1}^{j}, g_{4}=x_{1}^{t}, g_{5}=x_{1}^{k}, g_{6}=x_{2}^{r}$ temos

$$
\begin{gathered}
\left(g_{1} g_{2}, g_{3} g_{4}, g_{5} g_{6}\right)=\left(x_{1}^{i} x_{2}^{s}, x_{1}^{j} x_{2}^{t}, x_{1}^{k} x_{2}^{r}\right)=\left(x_{2}^{s+t}, x_{1}^{j}, x_{1}^{i}\right)\left(x_{1}^{j}, x_{2}^{t}, x_{2}^{2}\right)\left(x_{1}^{i}, x_{2}^{s}, x_{2}^{t}\right)\left(x_{2}^{t+r}, x_{1}^{k}, x_{1}^{j}\right)^{-1} \\
\left(x_{1}^{k}, x_{2}^{r}, x_{2}^{t}\right)^{-1}\left(x_{1}^{j}, x_{2}^{t}, x_{2}^{r}\right)^{-1}\left(x_{2}^{s+r+t}, x_{1}^{k}, x_{1}^{i+j}\right)\left(x_{1}^{k}, x_{2}^{r}, x_{2}^{s+t}\right)\left(x_{1}^{i+j}, x_{2}^{s+t}, x_{2}^{r}\right)\left(x_{2}^{s+t+r}, x_{1}^{j+k}, x_{1}^{i}\right)^{-1} \\
\left(x_{1}^{j+k}, x_{2}^{t+r}, x_{2}^{s}\right)^{-1}\left(x_{1}^{i}, x_{2}^{s}, x_{2}^{t+r}\right)^{-1}=z_{1}^{j(i r-k s)} z_{2}^{t(i r-k s)} .
\end{gathered}
$$

Então, o que mostramos foi

$$
\left(x_{1}^{i} x_{2}^{s}, x_{1}^{j} x_{2}^{t}, x_{1}^{k} x_{2}^{r}\right)=z_{1}^{j(i r-k s)} z_{2}^{t(i r-k s)} .
$$

Da equação 5.3 , temos

$$
\theta\left(x_{1}^{i} x_{2}^{s}, x_{1}^{j} x_{2}^{t}\right)=\theta\left(x_{1}^{i+j}, x_{2}^{s+t}\right)\left(x_{2}^{s+t}, x_{1}^{j}, x_{1}^{i}\right)\left(x_{1}^{j}, x_{2}^{t}, x_{2}^{s}\right)\left(x_{1}^{i}, x_{2}^{s}, x_{1}^{j}\right)\left(x_{1}^{i}, x_{2}^{s}, x_{2}^{t}\right),
$$

ou seja,

$$
\theta\left(x_{1}^{i} x_{2}^{s}, x_{1}^{j} x_{2}^{t}\right)=z_{1}^{-i j(s+t)} z_{2}^{t s(i+j)} .
$$

Com tudo isso mostramos um caso particular do teorema 5.0.8. Para $X\left\{x_{1}, x_{2}\right\}$ e $Y=\left\{z_{1}, z_{2}\right\}$ temos que $\mathbf{F}=\mathbb{Z} \times \mathbb{Z} \times \mathbb{Z} \times \mathbb{Z}$ admite a seguinte estrutura de $A$-loop comutativo livre, $\mathbf{F}=\mathbb{Z} \times \mathbb{Z} \ltimes_{\theta} \mathbb{Z} \times \mathbb{Z}$ onde

$$
\theta\left(\left(a_{1}, a_{2}\right),\left(b_{1}, b_{2}\right)\right)=\left(-a_{1} b_{1}\left(a_{2}+b_{2}\right), a_{2} b_{2}\left(a_{1}+b_{1}\right)\right)
$$


Sejam $\alpha=(a, x), \beta=(b, y)$ e $\gamma=(c, z)$ com $a=\left(a_{1}, a_{2}\right), b=\left(b_{1}, b_{2}\right)$ e $c=\left(c_{1}, c_{2}\right)$. Temos que

$$
(a, x)[(b, y)(c, z)]=[(a, x)(b, y)](c, z)
$$

se e somente se

$$
a_{1} b_{1} c_{2}=a_{2} b_{1} c_{1} \quad \text { e } \quad a_{2} b_{2} c_{1}=a_{1} b_{2} c_{2}
$$

Donde obtemos $\mathcal{N}_{\mu}(L)=\mathcal{N}_{\lambda}(L)=\mathcal{N}_{\rho}(L)=0 \times 0 \times \mathbb{Z} \times \mathbb{Z}$ e $\operatorname{assim} \mathcal{Z}(L)=\mathcal{N}(L)=$ $0 \times 0 \times \mathbb{Z} \times \mathbb{Z}$.

Além disso, desde que $(\alpha \beta) \gamma=(a b c, \theta(a b, c) \theta(a, b) x y z), \alpha(\beta \gamma)=(a b c, \theta(a, b c) \theta(b, c) x y z)$ e $(\alpha, \beta, \gamma)$ é o único elemento de $L$ tal que $(\alpha \beta) \gamma=[\alpha(\beta \gamma)](\alpha, \beta, \gamma)$ temos

$$
(\alpha, \beta, \gamma)=\left(0, \theta(a b, c) \theta(a, b) \theta(a, b c)^{-1} \theta(b, c)^{-1}\right)
$$

e, portanto, o associador $(\mathbf{F}, \mathbf{F}, \mathbf{F})$ está contido em $0 \times 0 \times \mathbb{Z} \times \mathbb{Z}$. Segue da definição do cociclo $\theta$ que $0 \times 0 \times \mathbb{Z} \times \mathbb{Z} \subset(\mathbf{F}, \mathbf{F}, \mathbf{F})$. $\operatorname{Logo}(\mathbf{F}, \mathbf{F}, \mathbf{F})=0 \times 0 \times \mathbb{Z} \times \mathbb{Z}$. 


\section{Referências Bibliográficas}

[GJM] GOODAIRE, E.G., JESPERS, E., MILIES, C.P., Alternative loop Rings, Mathematics Studies, 184, North Holland.

[Br] BRUCK, R. H., Contribuctions to the Theory of Loops, Transactions of American Mathematical Society, Vol. 60, No. 02, 1946, pp.245-354.

[BP] BRUCK, R. H., PAIGE, L. J., Loops Whose Inner Mappings Are Automorphisms, Annals of Mathematics, vol. 63, No. 2, 1956, pp.308-323.

[Gl-01] GLAUBERMAN, G., On Loops of Odd Oreder, Journal of Algebra, vol. 1, 1964 pp.374-396.

[Gl-02] GLAUBERMAN, G., On Loops of Odd Oreder II, Journal of Algebra, vol. 8, 1968 pp.393-414.

[JKV-01] JEDLICKA, P., KINYON, M., VOJTECHOVSKY, P. The Structure of Commutative Automorphic Loops to appear in Transactions of American Mathematical Society.

[JKV-02] JEDLICKA, P., KINYON, M., VOJTECHOVSKY, P. Constructions of Commutative Automorphic Loops to appear in Communications in Algebra. 